\title{
Abstracts of the Papers Presented at the 41st Annual Meeting of The American Society of Dermatopathology
}

\section{Westin Copley Place Boston, Massachusetts, USA October 14-17, 2004}

These abstracts were presented in oral or poster format at the 41st Annual Meeting of The American Society of Dermatopathology on October 14-17, 2004. They are listed on the following pages in alphabetical order by the first author's last name. 
IN SITU HYBRIDIZATION IS A VALUABLE DIAGNOSTIC TOOL IN CUTANEOUS DEEP FUNGAL INFECTIONS J.J. Abbott ${ }^{1}$, K.L. Hamacher ${ }^{2}$, A.G. Bridges ${ }^{2}$ and I. Ahmed ${ }^{1,2}$ Departments of Laboratory Medicine and Pathology ${ }^{1}$ and Dermatology ${ }^{2}$,

Mayo Clinic and Mayo Foundation, Rochester, MN, USA

Dimorphic fungal infections (histoplasmosis, blastomycosis, coccidiomycosis, and cryptococcosis) can occur in immunocompromised and healthy individuals. Cutaneous involvement is often secondary and may be the presenting sign of systemic disease. These ominous infections are frequently clinically indistinct and their prognosis is influenced by their timely diagnosis and treatment. Morphological differentiation between these organisms is not definitive and tissue cultures represent the diagnostic gold standard in current day practice. However, not uncommonly tissue cultures are not obtained and merely represent an afterthought in seemingly unsuspecting cases. Furthermore, when performed, they may take several days to weeks for completion. In situ hybridization (ISH) utilizing oligonucleotide probes directed against fungal ribosomal RNA represents a rapid and accurate assay for the diagnosis of dimorphic fungi in paraffin-embedded tissue sections. We present 7 patients in whom ISH both prospecitively and retrospectively confirmed the presence of a cutaneous infection (2 histoplasmosis, 2 blastomycosis, 1 coccidiomycosis, 2 cryptococcosis). In all of the skin sections analyzed, dimorphic fungi were morphologically apparent but not diagnostically discernable. In summary, ISH represents a valuable tool in the prompt diagnosis of cutaneous deep fungal infections.

\section{ADENOCARCINOMA OF MAMMARY-LIKE GLANDS OF THE VULVA \\ J.J. Abbott ${ }^{1}$ and I. Ahmed ${ }^{1,2}$ \\ ${ }^{1}$ Department of Pathology and \\ ${ }^{2}$ Department of Dermatology, Mayo Clinic and Mayo Foundation, Rochester, MN, USA}

Mammary-like glands are a poorly recognized structure of the normal vulvar skin. Adenocarcinomas occur rarely in these glands and represent a distinct clinicopathologic entity that must be distinguished from metastatic tumors. We present a case of a 51 -year-old patient with a long-standing nodule on her right labium majus. Excision with subsequent Mohs micrographic surgery revealed an infiltrating adenocarcinoma of the mammary-like glands involving the dermis and subcutis. We review all cases thus far reported (including the case presented herein) and identify important clinical and morphologic features of this tumor. A significant histopathological and immunohistochemical overlap between this neoplasm, sweat gland carcinomas, and invasive extramammary Paget's disease was recognized; suggesting the existence of a morphologic spectrum between these entities. Criteria for establishing the diagnosis of adenocarcinoma of mammary-like glands are reviewed, including the importance of identifying transition zones between normal mammary-like glands and malignant glands. We believe that this tumor is best categorized as a locally aggressive neoplasm with a low metastatic potential and death from disease is uncommon. The successful treatment of this case by Mohs micrographic surgery suggests that an alternate management approach may be adopted for these tumors, particularly in elderly patients.

\section{A NEW LOOK AT CUTANEOUS FEATURES OF SJOGREN'S SYNDROME}

P. Abrishami,

Department of Dermatology, University of Minnesota, Minneapolis, MN, USA
A 37-year-old woman with diagnosis of Sjogren's syndrome (SS) presented with asymptomatic non-palpable purpura of the lower extremities. Biopsy of a purpuric macule revealed a perivascular and focally nodular lymphocytic infiltrate with large numbers of plasma cells, seemingly around eccrine glands. There was no vasculitis. The histologic findings in the skin were strikingly similar to those of salivary, parotid, and other "secretory" glands affected in SS. The cutaneous manifestations of SS highlighted in textbooks include xerosis, annular erythema, small-vessel vasculitis, and pigmented purpura. This case illustrates that purpura in skin of patients with SS may be caused by a peri-eccrine plasma-rich infiltrate. The link between hypergammaglobulinemia, auto antibodies, plasma cells infiltration, and development of extranodal B-cell lymphoma in SS remains to be elucidated.

\section{MYCOBACTERIUM FORTUITUM FURUNCULOSIS IN PEDICURE RECIPIENTS; AN EPIDEMIC?}

P. Abrishami and C. Cockerell

Department of Dermatology, University of Texas Southwestern Medical Center, Dallas, TX, USA

Isolated cases and outbreaks of mycobacterial furunculosis among patients receiving pedicures have been reported. The transmission of the mycobacteria likely occurs while soaking the legs in a whirlpool foot bath prior to the pedicure. Oils are often applied to the legs following the foot bath. Typically, patients present with multiple papules and pustules below the knees that, after several weeks or months, may become large, tender, and fluctuant furuncles. Histology usually demonstrates a suppurative folliculitis with or without associated suppurative granulomatous inflammation. We present a culture-proven case of M. fortuitum furunculosis in a patient after receiving whirlpool leg soaking and pedicure in a foot salon. We also illustrate several other highly suspected cases in which cultures failed to grow the organism. It is important for clinicians and pathologists to recognize the gross and histological patterns of this underreported condition as cultures, particularly swabs, often fail to grow the organism.

\section{NEPHROGENIC FIBROSING DERMOPATHY}

M. Mubashir Ahmed, Richard Kim and Li Ma

Department of Pathology, Louisiana State University Health Sciences Center- Shreveport, LA, USA

Nephrogenic fibrosing dermopathy is a rare, recently described fibrotic skin condition that primarily affects patients with a history of renal disease.We report 2 cases of nephrogenic fibrosing dermopathy and review clinical and histopathologic features with differential diagnosis and more.Case \#1 was a 64-year-old black male with chronic renal insufficiency which progressed to end stage renal disease requiring hemodialysis. After two days, he developed blisters on both his hands. By a month, his blister lesions resolved leaving scaly skin with some skin thickening and tightening in the hands and forearms. Over the course of one month, skin over above-mentioned areas began to harden and stiffen. His fingers began to coalesce and contract resulting in fixation of hand grip. He also developed flexing contracts at the elbows bilaterally. Skin biopsy revealed the diagnosis of nephrogenic fibrosing dermopathy with giant cells reaction. Case \#2 was a 44year-old black male with a hx of nephropathy. He eventually developed end stage renal disease requiring hemodialysis. One month after, he was found to have thickened, sclerotic appearing skin involving the upper back, hands, and forearms with flexing contracts of the all the small joints. Histopathology was most consistent with nephrogenic fibrosing dermopathy. 
BASAL GELL CARCINOMA WITH MATRICAL DIFFERENTIATION IN A TRANSPLANT PATIENT: A CASE REPORT AND REVIEW OF THE LITERATURE

F. Ali, A. Brown, L. Gottwald and J. Thomas

Departments of Pathology and Dermatology, Medical College of Ohio, Toledo, OH, USA

Background: Shadow cells are characteristic of pilomatricoma, a distinct neoplasm of hair matrix differentiation. Shadow cells within an otherwise classic basal cell carcinoma (BCC) has been referred to as "BCC with matrical differentiation". We present a case of BCG with matrical differentiation in a transplant patient. To our knowledge, none have been reported arising on the background of immunosuppression. Methods: A 58-year-old male cardiac transplant patient had a left hand nodule, which was excised and submitted for routine histologic review. Results: The lesion revealed multiple basaloid tumor masses. In some areas, there was peripheral palisading with stromal retraction artifact, typical of basal cell carcinoma, extending into the deep reticular dermis. The tumor also contained a population of shadow cells, similar to those in pilomatricoma, with basaloid cells in the periphery. Trichohyaline granules were identified in many of the tumor cells. These granules are a hallmark of follicular matrix differentiation. Mitoses were rare. There was no evidence of an infiltrating growth pattern. Conclusion: Basal cell carcinoma with matrical differentiation is a rare subtype of basal cell carcinoma featuring shadow cells, such as those typically seen in pilomatricoma. This tumor has not yet been reported in an immunosuppressed transplant patient.

\section{CUTANEOUS LARVA MIGRANS}

A. Allan ${ }^{1}$ and H. Oriba ${ }^{2}$

${ }^{1}$ Pathology Services, Inc., Cambridge, MA, and

${ }^{2}$ Private Practice, Tampa, FL

Cutaneous larva migrans is the most common tropically acquired dermatosis caused by larvae of various nematodes, which penetrate the skin and subsequently migrate, causing serpiginous lesions and pruritus. The diagnosis is usually made clinically. While a biopsy taken just ahead of the leading edge of a lesion is felt to enhance the yield of a positive biopsy, the larvae are migratory and thus rarely identified on histologic sections. We present a case of cutaneous larva migrans in which the larval forms are well demonstrated both in a horizontal and longitudinal orientation. A 75-year old man presented with intractable pruritus and multiple erythematous plaques on his back, poorly responsive to topical steroids. The diagnosis was made on biopsy. Associated with a perivascular lymphocytic infiltrate with eosinophils, a cross section of a larval form was identified within the epidermis. In a second biopsy, the entire organism was present within a sebaceous gland. This case clearly demonstrates the rarely identified larval forms of cutaneous larva migrans.

\section{SEBACEOUS CARCINOMA OF THE EYELID: ANALOGY TO EXTRAMAMMARY PAGET'S DISEASE \\ J.B. Lee and I. Amir \\ Thomas Jefferson University, Philadelphia, PA, USA}

We present a 69-year-old woman with a sebaceous carcinoma of the left upper eyelid, which originated from the Meibomian gland of the tarsal plate. The intraepidermal extension involved not only the conjunctival epithelium but also the overlying skin with subsequent extension into the dermis. The discussion will include diagnostic, both clinical and microscopic, considerations along with comparison to mammary Paget's disease. Just as mammary Paget's disease, which originates from the glands in the dermis eventuating in the seeding of the overlying epidermis, so too does sebaceous carcinoma of the eyelid. Accordingly, just as mammary Paget's disease is often misdiagnosed as an inflammatory condition, so too is sebaceous carcinoma.

\section{AXILLARY METASTASIS OF MALIGNANT MELANOMA MIMICKING A BREAST CARGINOMA}

A. Andea, E. Satter, J.R. McEvoy, K. Williams, K. Mikhitarian and J. Metcalf

Department of Pathology, Medical University of South Carolina, Charleston, SC, USA

We describe here the case of a female patient with an otherwise classic invasive melanoma of the upper extremity, demonstrating the typical immunohistochemical profile, who presented three years later with massive ipsilateral axillary node metastases. The metastatic tumor had morphologic features similar to the primary melanoma, however at immunohistochemical level the tumor cells not only gained expression of pan-cytokeratin (AE1/AE3) but also lost expression of all standard melanoma markers (S100, Melan-A, HMB-45, MIT-1, and tyrosinase) thus mimicking a poorly differentiated mammary carcinoma. Additional stains reveal expression of Vimentin, NSE, c-kit, CD10 and negativity for CK7, CK20, BR2, ER/PR, mucin, and LCA. To rule out a breast carcinoma, real-time RT-PCR was performed on a lymph node using six breast cancer-associated genes (>99\% sensitivity), which was also negative. Clinical work-up of the patient failed to reveal abnormal masses in the breast or elsewhere. The clinical history, histological appearance, immunohistochemical profile (including negative CK7 and positive NSE, c-kit and CD10) as well as a negative RT-PCR renders almost null the possibility of a breast carcinoma and supports a diagnostic of melanoma in this case. Cases like ours may be easily misclassified unless a concerted approach is used to reach the correct diagnosis.

\section{METASTATIC BASAL GELL GARGINOMA WITH NEUROENDOCRINE DIFFERENTIATION OR MERKEL CELL CARGINOMA?}

A. Andea, J. Mefcalf, E. Satter and V. Adsay

Department of Pathology, Medical University of South Carolina, Charleston, SC, Wayne State Unversity, Detroit, MI

We present here a case of basal cell carcinoma (BCC) with neuroendocrine features that has transformed into a high-grade neuroendocrine carcinoma with various morphologic features of Merkel cell carcinoma (MCC). A 54-year-old white female was treated for a $\mathrm{BCC}$ of the right thigh. Pathologic examination revealed an otherwise classical BCG that demonstrated granular positivity for chromogranin. Six years later the patient developed a right inguinal lymphadenopathy diagnosed as metastatic BCC with squamous changes. The metastatic BCG showed partial peripheral palisading and a trabecular pattern. Two years later the patient underwent a right nephrectomy due to obstruction of the right ureter by metastatic BCG. After another four years the patient came back with extensive involvement of the appendiceal wall and right ovary by a diffusely infiltrating metastatic basaloid and trabecular carcinoma. This time the tumor had many histologic features of MCG and showed strong positivity for chromogranin and also for CK20 and NSE. Electron microscopy revealed neurosecretory granules. This case is an example of a chromogranin positive basal cell carcinoma of the skin, which transformed during multiple recurrences into a high grade neuroendocrine carcinoma with features of Merkel cell tumor, demonstrating the potential for cross differentiation among skin tumors. 
NUMEROUS SEBACEOUS NEOPLASMS IN A PATIENT WITHOUT VISCERAL GARCINOMAS

G. Ang, C. Campanelli, F. Ringpfeil, G. Webster and J. Lee Dept. of Dermatology, Jefferson Medical College, Philadelphia, PA, USA

We present a 58-year-old Caucasian man who spawns sebaceous neoplasms with a widely varying histopathologic pattern, the true nature of which were difficult to determine in some of the cases. In a 10-year period, the patient developed over 20 lesions that varied in size, shape, and location. The patient denied a family history of either similar cutaneous lesions or internal malignancies that would fulfill the criteria of Hereditary Nonpolyposis Colorectal Cancer (HNPCG). Vigilant screening failed to reveal visceral carcinomas. The histopathologic findings of the neoplasms as well as the results of tests for microsatellite instability(MSI) in various tumors and the immunohistochemical staining for the mismatch repair genes (MMR) MSH2 and MLH1 will be discussed. Our patient has been maintained on low-dose isotretinoin since 1995.

\section{D2-40 EXPRESSION IN HEMANGIOMAS AND} LYMPHANGIOMAS: SPECIAL REFERENCE TO ITS DIFFERENTIAL UTILITY BETWEEN KAPOSIFORM HEMANGIOENDOTHELIOMA AND ACQUIRED TUFTED ANGIOMA

E. Arai, A. Kuramochi, M. Shimizu and T. Hirose

Departments of Pathology and Dermatology, Saitama Medical School, Saitama, Japan.

Recent investigations have demonstrated the utility of D2-40 as a marker for lymphatic endothelium and can be used for formalin-fixed and paraffin-embedded materials. We studied 13 capillary hemangiomas: kaposiform hemangioendothelioma $(\mathrm{KH}, 2)$, acquired tufted angioma (ATA, 5), juvenile hemangioma (JH, 2), granuloma pyogenicum (GP, 4); 6 other hemangiomas: cavernous hemangioma (3), arterio-venous hemangioma (1), angiokeratoma (1), epithelioid hemangioma (1); Kaposi's sarcoma (1); 12 lymphangiomas: lymphangioma circumscriptum (6), deep type of cutaneous lymphangioma (6). D2-40 monoclonal antibody, endothelial markers (CD31, CD34, Factor VIII related antigen) and juvenile hemangioma-associated marker GLUT-1 were applied. D2-40 was immunopositive for the peripheral area of proliferative capillaries and negative for surrounding dilated vessels in $\mathrm{KH}$, but positive for surrounding dilated vessels and negative for tufted proliferative capillaries in ATA. KH and ATA were all positive for endothelial markers and negative for GLUT-1. Other hemangiomas were negative for D2-40. Eight of 12 cases were positive for lymphangiomas. Based on these results, D2-40 is a useful additional immunostain for the discrimination of $\mathrm{KH}$ and ATA. KH and ATA may originate from different stage of both the lymphatic and blood vessel endothelial lineages.

\section{FOLLICULAR ERUPTION IN A CHILD WITH ALAGILLE SYNDROME}

A. Armstrong, V. Liu and J. Strasswimmer

Department of Dermatology, Massachusetts General Hospital, Boston, MA, USA

A 17 year-old boy with a history of Alagille syndrome presented with three-months duration of spontaneous eruption of multple non-pruritic, non-painful papules on the extremities. He also reported seeing "dark spots" at night. On physical examination, the patient was jaundiced and appeared smaller than his age. The skin examination revealed numerous $2-8 \mathrm{~mm}$ follicular, keratotic papules on the exten- sor surfaces of the patient's arms and legs. H\&E preparation of the skin biopsy revealed distention of the upper portion of the follicles with prominent keratotic plugging, as well as focal follicular perforation and acute inflammation. Laboratory examination revealed a vitamin A level of 11, carotene level of less than 2, and retinol binding protein of 2.1. The patient was diagnosed with phrynoderma and initiated on vitamin A therapy. After a two-months course of vitamin A therapy, his serum vitamin A increased to 67, and he had dramatic improvement of the follicular eruption. This is a fascinating case of phrynoderma in a child with biliary dysfunction. The presence of perforating folliculitis on histology is curious, as there are less than 10 documented cases of perforating folliculitis in phrynoderma. This case illustrates the intriguing clinical and pathological presentations of phrynoderma.

\section{NEUTROPHILIC DERMATOSIS SECONDARY TO IMATINIB THERAPY IN A PATIENT WITH GML: A CASE REPORT}

S.J. Ayirookuzhi, L Ma, P. Ramshesh and G. Mills

Department of Pathology, Louisiana State University Health Sciences Center at Shreveport, Shreveport, LA, USA

A 53 year old woman with CML in the chronic phase received therapy with Imatinib on two separate occasions. Both occasions were followed by the development of an abrupt onset of skin lesions characterized by painful and tender erythematous plaques and nodules on the upper extremities that subsequently resolved with hyperpigmentation. The patient was also found to have pleural and pericardial effusions. She had a WBC count of 57,250 cells per $\mathrm{mm}^{3}$, a platelet count of 740,000 per $\mathrm{mm}^{3}$ and a single temperature spike of $100.4^{\circ} \mathrm{F}$. Over the course of a week the WBC count dropped to 51,520 cells per $\mathrm{mm}^{3}$ but the percent of mature neutrophils rose to $55 \%$. Imatinib was discontinued and steroid therapy led to resolution of the skin lesions with residual pigmentation. A skin biopsy was obtained that showed neutrophilic dermatosis with epidermal sparing consistent with Sweet's syndrome but with atypical features such as extension into deep dermis and subcutaneous fat. Lab work up including blood cultures and bacterial, fungal and mycobacterial cultures of the skin as well as special stains of the skin biopsy specimen failed to reveal any microbiological etiology.

\section{TISSUE EOSINOPHILIA AS AN INDICATOR OF DRUG- INDUCED LEUKOCYTOCLASTIC VASGULITIS: A CLINICOPATHOLOGIC INVESTIGATION \\ S. Bahrami, J. Malone, K. Webb and J.P. Callen \\ Department of Internal Medicine, Division of Dermatology and Department of Pathology, University of Louisville School of Medicine, Louisville, KY, USA}

Cutaneous leukocytoclastic vasculitis (LCV) may be idiopathic, druginduced, related to infection, or may occur in patients with autoimmune disorders. Our objective was to determine whether tissue eosinophilia is a reliable indicator of a drug-induced etiology in the setting of LCV. Thorough chart investigation of 63 patients with LCV was performed with division of patients into drug-induced and non-drug-induced groups. Corresponding histopathologic material was reviewed by a dermatopathologist blinded to the etiologic associations. Â An eosinophil score was tabulated by averaging the number of eosinophils in 10 high-power fields (400x) and dividing by the density of inflammation. Statistical analysis was performed using the Wilcoxin rank sums test. Mean eosinophil scores in the drug-induced $(\mathrm{n}=16)$ and non-drug-induced $(\mathrm{n}=47)$ groups were 5.20 and 1.05 respectively $(\mathrm{p}=0.0126)$. Vascular fibrin deposition and epidermal 
changes were present in both groups. Clinical evidence of systemic vasculitis was present in $6 \%(1 / 16)$ of the drug-induced group vs. $38 \%$ $(18 / 47)$ of the non-drug-induced cases. This study establishes tissue eosinophilia as a reliable indicator of drug induction in the setting of LCV. Furthermore, this information may be useful for guiding management decisions, especially in settings of unclear etiology.

\section{THE HISTOLOGIC DIFFERENCES BETWEEN NEUROFIBROMAS IN PATIENTS WITH NEUROFIBROMATOSIS, TYPE I AND SPORADIC NEUROFIBROMAS}

N.J. Ball ${ }^{1}$ and G. Tanhuanco-Kho ${ }^{2}$

Departments of Pathology and Medicine (Dermatology) ${ }^{1}$, University of British Columbia and Vancouver General Hospital, Vancouver, BC; and Department of Lab. Medicine ${ }^{2}$, Royal Jubilee Hospital, Victoria, BC, Canada.

Neurofibromatosis is associated with cutaneous melanin pigmentation, but an association with ordinary melanocytic nevi has not been described. This retrospective case-control study was designed to see if neurofibromas in patients with Neurofibromatosis, Type I (NF-1) differ from sporadic neurofibromas (SN) in their incidence of associated melanocytic nevi and other histologic features. Slides from 114 NF-1 were compared with 112 SN. Lentiginous melanocytic nevi were identified over 13 NF-1 (11\%) but no SN ( $p=0.00019$, Fisher's test). Compared with other NF-1, NF-1 with nevi were more frequently associated with melanocytic hyperplasia, honeycomb infiltration of the subcutis $(p<0.03$, Fisher's test), and a greater number of biopsies $(p=0.04$, t-test). Compared with SN, NF-1 were more frequently associated with melanocytic hyperplasia, lentigo simplex-like changes, honeycomb infiltration of the subcutis $(p<0.001$, Chi squared test), plexiform neurofibroma and epidermolytic hyperkeratosis ( $\mathrm{p}<0.03$, Fisher's test). SN more frequently presented as a polyp or papule $(\mathrm{p}<0.009)$. Sebaceous hyperplasia (present in $14 \%$ of cases), dermal elastosis $(9 \%)$, lipomatous neurofibroma (8\%), epithelial cysts $(4 \%)$, and keratin granulomas or folliculitis (3\%) were equally common in NF-1 and SN. This study suggests that there may be a fundamental difference in the potential for melanocytic proliferation in NF-1 compared with $\mathrm{SN}$.

\section{HISTOLOGIC VARIANTS OF FIBROUS PAPULE}

C. Bansal, D. Stewart, A. Li and C. Cockerell

Department of Dermatology, Division of Dermatopathology, University of Texas Southwestern Medical Center, Dallas, TX, USA

Fibrous papule (FP), synonymous with angiofibroma, is a common lesion that is often biopsied to exclude carcinoma. Clinically, FPs are small, dome-shaped, solitary, flesh colored papules arising on the nose and face of adults. Histologically, FPs are characterized by a domeshape, fibrotic collagenized stroma, sparse inflammatory cell infiltrate, and variably increased and dilated vascular spaces. The vast majority of FPs are readily diagnosable based on these clinical and histologic criteria. Rare histopathologic variants have been reported. Recognition of these variants may avoid misdiagnosis of this common benign lesion. We illustrate four rare variants of FP that can cause diagnostic uncertainty including: hypercellular, clear cell, pigmented, and pleomorphic variants. Cellular FP has a dense infiltrate of round fibroblasts that have a nevoid appearance. Clear cell FP has a proliferation of round clear cells, some with slightly foamy cytoplasm, resembling histiocytes or clear epithelial cells. Pleomorphic FP demonstrates bizarre, stellate fibroblasts like those seen in pleomorphic fibroma. Pigmented FP has prominent melanocytic hyperplasia and dermal melanophages that can be confused with a melanocytic lesion. It is important to recognize these unusual variants of FP in order to accurately diagnose this common benign lesion and avoid over treatment or rebiopsy.

\section{MYCOBACTERIA OTHER THAN MYCOBACTERIUM TUBERGULOSIS ARE NOT PRESENT BY PGR IN ERYTHEMA INDURATUM/NODULAR VASGULITIS IB Bayer-Garner, MD Cox, MA Scott, BR Smoller, Marshfield Clinic, Marshfield WI, John L. McClellan \\ Memorial Veterans Hospital \& University of Arkansas for Medical Sciences, Little Rock AR}

Erythema induratum/nodular vasculitis is characterized by recurrent crops of tender edematous nodules on the lower legs. lobular panniculitis with granulomatous inflammation, vasculitis, focal necrosis and septal fibrosis is present. Mycobacterium tuberculosis DNA has been detected in some lesions by polymerase chain reaction (PCR). Ten cases of erythema induratum/nodular vasculitis were found. $\mathrm{H}$ \& $\mathrm{E}$ stained slides were reviewed. PCR assays for $\mathrm{M}$ tuberculosis and mycobacteria other than tuberculosis (MOTT) was carried out. PCR did not reveal mycobacterial DNA, with positive controls indicating the reliability of the assays. Among the MOTT, cutaneous infections are most commonly caused by M. marinum. Subcutaneous tuberculoid granulomas may be seen with M. kansaii, M. marinum, M. scrofulaceum, and M. avium complex. M. gordonae and M. malmoense rarely cause cutaneous infections. M. szulgai, M. simiae, M. gastri and M. asiaticum are probably not cutaneous pathogens. MOTT DNA was not found $\hat{A}$ in erythema induratum/nodular vasculitis. Erythema induratum/nodular vasculitis has diverse etiologies with varying pathogeneses leading to similar histologic changes. The cases analyzed may have not have had an infectious etiology. However, in erythema induratum/nodular vasculitis, performance of Â PGR for MOTT as well as Mycobacteria tuberculosis complex may still be beneficial, particularly in cases from immunocompromised hosts.

\section{EXPRESSION OF ACTIVATED MAPK, AKT AND G-KIT IN MELANOCYTIC LESIONS \\ C. Bengana, A.H. Diwan, K.B. Kim, P.S. Zhang and V.G. Prieto Department of Pathology, UT MD Anderson Cancer Center, Houston, TX, USA}

Background: MAPK, AKT, and c-Kit are major signaling molecules. This study evaluates the expression of the activated forms of these molecules in melanocytic lesions. Design: Tissue microarrays were constructed using formalin-fixed, paraffin-embedded archival tissue from 14 benign nevi (BN), 16 dysplastic nevi (DN), 18 malignant melanomas (MM), and 25 metastatic malignant melanomas (MMM). Two control cases (nevus and melanoma) were included in all three microarray blocks. Immunohistochemical analysis of p-MAPK, p-AKT and p-c-kit was performed using standard technique. Both the intensity and the number of labeled cells were recorded in a semiquantitative fashion. Results were analyzed using Chi-square test, with p Results: c-kit expression was highest in BN compared to DN, $\mathrm{MM}$ and MMM that showed similar expression. Among MMM, the visceral metastases had the strongest c-kit expression. p-AKT showed decreased expression with progression of the melanocytic lesions. The differences for p-c-kit and p-AKT were highly significant (0.04 and 0.0001 respectively). There was no statistically significant difference in expression of p-MAPK. Conclusion: Since the observed changes apply to the activated forms of AKT and c-kit these molecules are likely involved in melanoma progression. These findings may help determine the applicability of targeted therapies such as gleevec. 
POST-IRRADIATION VASCULAR PROLIFERATIONS: POTENTIAL MIMIC OF KAPOSI'S SARCOMA

J. Bennett and J. McNiff

Departments of Pathology and Dermatology, Yale University School of Medicine, New Haven, CT, USA

A variety of benign and malignant cutaneous vascular lesions occur in irradiated skin. We report two cases of vascular lesions that developed on the breast within a year after radiation therapy for breast carcinoma. Both cases mimicked Kaposi's sarcoma (KS) histologically. We compare these with one case of post-irradiation angiosarcoma and 3 cases of acquired progressive lymphangioma (APL) unrelated to radiation therapy. The post-irradiation vascular lesions showed slitlike vessels dissecting through superficial dermal collagen, leading to an initial diagnosis of KS by one contributing pathologist. Immunohistochemically, the vessels in both cases expressed CD31 and CD34, stained only partially for actin, and had a low Ki67 proliferation index. Neither case expressed HHV8. The post-irradiation angiosarcoma occurred three years after radiation, and also exhibited a focal slit-like growth pattern resembling KS. However, there was marked cytologic atypia, a high proliferation rate, and negative staining for HHV8. The APL were characterized by more ectactic vascular spaces and deeper involvement of the dermis than was present in the post-irradiation lesions. These cases also failed to stain for HHV8. Recognition of the phenomenon of post-irradiation vascular proliferations is important, especially because such lesions may resemble $\mathrm{KS}$, yet invariably follow a benign clinical course.

\section{IMMUNOHISTOCHEMICAL EXPRESSION OF CUTANEOUS LEIOMYOSARCOMA \\ P. Bhattacharjee and R. Lazova \\ Department of Dermatology, Yale University School of Medicine, New Haven, GT, USA}

Immunohistochemistry plays a vital role in distinguishing cutaneous leiomyosarcoma (CLMS) from other spindle cell neoplasms. Recently, several new immunohistochemical markers of smooth muscle differentiation (calponin, h-caldesmon) have shown greater utility in the diagnosis of CLMS. We compared the expression of various traditional and novel immunohistochemical markers in CLMS. Thirteen cases of CLMS were immunostained with a panel of antibodies (SMA, MSA, desmin, vimentin, S100, cytokeratin, NSE, HMB-45, CD117, procollagen, h-caldesmon and calponin. Immunostaining was graded from 0 to $4+$ based on the percentage of positive staining. All 13 cases of CLMS showed positive staining with SMA, MSA and h-caldesmon. 12 cases showed positive staining with desmin, calponin, vimentin and NSE. 8 cases showed positive staining with CD117 and procollagen. 4 and 3 cases showed focal positive staining for S100 and cytokeratin. All cases were HMB-45 negative. All 13 cases exhibited greater than $50 \%$ staining with SMA and MSA. 11 cases were strongly positive $(>50 \%)$ for calponin and h-caldesmon, while only 8 cases were strongly positive for desmin. Our study finds no significant difference between traditional and novel smooth muscle immunostains. We conclude that a panel of immunohistochemical stains should be employed to differentiate CLMS from other spindle cell neoplasms.

\section{HERPETIC FOLLICULITIS IS USUALLY A CONSEQUENCE OF VARICELLA ZOSTER VIRUS INFECTION}

Blair J, Kutzner H and McCalmont TH

Friedrichshafen, Germany, and University of California, San Francisco, CA, USA
Skin biopsies of 8 patients diagnosed with herpetic folliculitis by light microscopy were retrieved from the files of the UCSF Dermatopathology Service. Clinical and microscopical features were reviewed and tabulated, and PCR analysis was employed to seek DNA sequences specific for herpes simplex virus (HSV) and varicella zoster virus (VZV). The study group included 4 women and 4 men, ages 15 to 54 . Five patients $(62 \%)$ were immunosuppressed, with underlying conditions including HIV infection, leukemia, rheumatoid arthritis, and lupus erythematosus with polyarteritis nodosa. Microscopically, herpetic cytopathic changes involved the isthmus in $7 / 8$ cases $(87 \%)$, and involved the sebaceous apparatus in $4 / 8$ cases $(50 \%)$. Herpetic viropathic changes were not found within eccrine epithelium. A moderate to dense perifollicular infiltrate, comprised mostly of lymphocytes, was evident in 7/8 cases (87\%). After PCR expansion of genetic material extracted from the original paraffin blocks, VZVspecific DNA sequences were detected in $8 / 8$ cases. We conclude that herpetic folliculitis is a consequence of VZV infection. Because follicular herpetic infection is often accompanied by a dense perifollicular lymphoid infiltrate, the microscopical presentation can simulate inflammatory skin diseases such as lupus erythematosus. Level sections may be required for a specific diagnosis to be made.

\section{THE HISTOPATHOLOGY OF SUBCUTANEOUS MINOCYCLINE HYPERPIGMENTATION}

A.R. Bowen and T.H. McCalmont

Departments of Pathology and Dermatology, University of California, San Francisco, San Francisco, CA, U.S.A

Hyperpigmentation associated with prolonged minocycline use is well documented and affects diverse organ systems including skin, thyroid, heart valves, bone, sclerae and teeth. The histopathology of cutaneous minocycline pigment demonstrates brown-black granules lining elastin fibers in the upper dermis and occurring within macrophages along vessels and eccrine units in the dermis. The nature of the pigment can be confirmed by concurrent positive staining with both Fontana-Masson and Perls's stains. The subcutis may also be involved, however the specific subcutaneous findings in minocycline hyperpigmentation have not been described. We present the pathological findings in four cases of minocycline hyperpigmentation with subcutaneous involvement. Course black/brown granules were seen in macrophages particularly around vessels in the subcutis but also within septae and between lipocytes. An unusual feature noted in the subcutis and deep dermis was the presence of green-gray, flocculent, non-refractile, non-polarizable globules within macrophages and multinucleated giant cells. These measured between $0.005-0.1 \mathrm{~mm}$ in diameter and did not stain for melanin or iron. Two of four exhibited lipomembranous changes which were also involved by pigment. This is the first description of green-gray globules and pigmented lipomembranous changes in subcutaneous minocycline pigmentation, unique findings that provide additional clues to the diagnosis.

\section{PSEUDOXANTHOMA ELASTICUM (PXE)-LIKE FIBERS IN PATIENTS WITHOUT PXE}

A.R. Bowen, C. Gotting, P.E. LeBoit and T.H. McCalmont Departments of Pathology and Dermatology, University of California, San Francisco, San Francisco, CA, U.S.A. and Institut fur Laboratoriums- und Transfusionsmedizin, Bad Oeynhausen, Germany

Pseudoxanthoma elasticum (PXE) is an inherited disorder characterised by progressive calcification of elastic fibers in the skin, eyes and cardiovascular system. Skin lesions show distinctive thickened, 
fragmented elastic fibers in the reticular dermis. PXE-like fibers have not been described in patients without PXE. We describe 12 patients without known PXE who demonstrated the incidental finding of small numbers of PXE-like fibers in association with the following conditions: lipodermatosclerosis, granulomatous dermatitis, lichen sclerosus, morphea profunda, erythema nodosum, septal panniculitis, basal cell carcinoma and fibrosing dermatitis NOS. The biopsies were almost exclusively from the lower extremities of elderly women (mean age 73.8). The affected gene in PXE has been recently described as ABCC6 which codes for a member of the ATP-binding cassette (ABC) family of proteins. The c. $3421 \mathrm{C}>\mathrm{T}$ mutation has been associated with a high risk of coronary artery disease. We describe a novel assay for this genotype employing real-time PCR of paraffin embedded skin biopsies. Four patients with PXE-like fibers were tested in this fashion and did not carry this mutation. Our series demonstrates that light microscopic findings associated with PXE are not specific, as PXE-like fibers can be observed in patients without clinical or genetic evidence of the disease.

\section{LEUKOGYTOCLASTIC VASCULITIS PRESENTING IN ASSOCIATION WITH A SPINDLE CELL SARCOMA OF THE HEART. A PARANEOPLASTIC SYNDROME? \\ M. Braman, B. Santmyire-Rosenberger, E. Dugan, E. Brun and Z. Wang}

The rare coexistence of vasculitis and malignancy is intriguing, if not completely understood. Overall, malignancy is found in approximately $5 \%$ of patients who present with vasculitis. Leukocytoclastic vasculitis (LCV) is reported as a paraneoplastic event primarily in association with hematologic malignancies. Rarely, LCV has occurred concomitant with solid tumors, including sarcomas. We report a case of a 62-year-old African American woman who presented with LCV in association with an occult cardiac sarcoma. Past medical history includes left sided breast cancer treated with chemotherapy and radiation. She presented with worsening of a persistent, non-pruritic rash involving her lower extremities, diagnosed as LCV on skin biopsy. Within four months she developed congestive heart failure, and a right ventricular mass was identified. Heart biopsies revealed a high grade cardiac sarcoma. Within 2-3 days, she experienced an exacerbation of her vasculitis, accompanied by hemorrhagic bullae. Vasculitis is considered to be a paraneoplastic syndrome if a temporal relationship can be established with a co-existing malignancy. Here, a clear cut temporal relationship between coexistence, and intervention related exacerbation, is well documented. Temporal exacerbation of the LCV after cardiac biopsies suggests activation of the immune system in response to antigenic determinants within the cardiac tumor.

\section{A CASE REPORT OF MULTIPLE PIGMENTED PERINEAL HIDROCYSTOMAS \\ G. Breen}

Dept. of Pathology, Roger Williams Medical Center, Providence, RI, USA; A. Chang, Dept. of Dermatology, New England Medical Center, Boston, MA, USA; C. Wilkel, Depts. of Dermatology and Pathology, Roger Williams Medical Center, Providence, RI

Apocrine hidrocystomas occur most commonly as solitary nodules on the face and neck. Case reports of patients with multiple lesions on the face, particularly in the periorbital region, have been described. They present as cystic, translucent, blue or brown papules or nodules. The color is attributed to the Tyndall effect, as pigment such as melanin or hemosiderin has rarely been demonstrated within the lesions. We present a 43-year-old Caucasian female with over thirty blue-gray macules on her perineum and vulva. The patient was unaware of the lesions though she noted perineal and inguinal pruritus after exercise. Punch biopsy showed a cluster of cysts within a loose stroma, located in the deep dermis. The cysts were lined by a single layer of flattened cells with eosinophilic cytoplasm and focal areas of papillation and decapitation secretion. A peripheral layer of myoepithelial cells was present. The epithelium contained brown pigment confirmed to be melanin by Fontana-Masson stain. Iron stain was negative. GCDFP-15 (gross cystic disease fluid protein) labelled the epithelial cells. S-100 and Mart-1 immunohistochemical stains were negative. Unusual features of this case include the anatomic site, number of lesions, and demonstration of melanin as the cause of the clinical pigmentation.

\section{HISTOLOGIC EVALUATION OF MUCOCUTANEOUS GRAFT VERSUS HOST DISEASE IN NONMYELOABLATIVE HEMATOLOGIC STEM CELL TRANSPLANTS \\ A.T. Bridge, R.P. Nelson, J.E. Schwartz, G.W. Mirowski and S.D. Billings \\ Departments of Pathology, Dermatology, Hematology and Oncology, Indiana University School of Medicine, Indianapolis, IN, USA}

Nonmyeloablative hematologic stem cell transplantation (HSCT) is a recent alternative to myeloablative HSCT in the treatment of hematologic malignancies. Graft versus host disease (GVHD) remains as a complication of both types of HSCT. Recently the clinical phenomenon of late-onset (>100 days) acute GVHD has been described following nonablative HSCT. However, there has been no relevant description of the histologic features of GVHD in this setting. We reviewed clinical and pathologic features of 28 cases of acute and chronic GVHD following nonablative HSCT at out institution between 2000 and 2003. Our results support the observation of late onset acute GVHD in nonablative HSCT. The mean onset of acute GVHD was 109.8 days (range 8-410 days). Furthermore, we identified a subset of patients $(8 / 23)$ in the acute GVHD group with composite histologic features of both acute and chronic GVHD. This subset was more likely to develop chronic GVHD as compared to patients with only classic acute GVHD, $87.5 \%$ versus $25 \%$, respectively $(\mathrm{p}<0.01)$. Composite histologic features could serve a a predictor of an increased risk for the development of chronic GVHD. These patients may potentially benefit from more aggressive treatment than patients with histologic features of solely acute GVHD.

\section{INTRAVASCULAR LYMPHOMA WITH NK CELL IMMUNOPHENOTYPE}

N. Brinster, S. Granter, G. Pinkus and D. Miller

Departments of Pathology and Dermatology, Brigham and Women's Hospital, Boston, MA, USA

Intravascular Lymphoma is a rare extranodal large cell lymphoma characterised by a neoplastic proliferation of lymphoid cells in small vessel lumina typically involving the skin and central nervous system. The malignant cells are most commonly of B cell origin, although rare $\mathrm{T}$ cell variants have been described. We report a rare case of intravascular lymphoma with an NK cell phenotype. The patient is a 65 year-old man with a 5 year history of hypogammaglobulinemia and a six month history of tinea corporis involving the legs. Following topical antifungal treatment.asymptomatic erythematous plaques were noted on the lower extremities. A skin biopsy revealed intravascular thrombi with atypical malignant lymphoid cells which were immunoreactive for: CD3, CD2, LCA, CD56, perforin, granzyme $\mathrm{B}$, and TIA-1. There is one description of an NK-like intravascular 
lymphoma in the literature and one of the authors (G.P.) has recently described two patients with intravascular lymphoma of definitive NK cell type (submitted for publication). Intravascular lymphoma is an aggressive malignancy with a poor prognosis. Recognition of this newly described variant will help to elucidate our understanding of its pathogenesis and natural history.

\section{CUTANEOUS LEIOMYOSARCOMA RESEMBLING A SKIN TAG \\ T. Brown, J.M. Junkins-Hopkins and D. Grossman* \\ Department of Dermatology, University of Pennsylvania, Philadelphia, PA, USA, *Clinical Associate, Department of Dermatology, University of Pennsylvania}

Cutaneous leiomyosarcoma typically presents as solitary, well-circumscribed, firm plaques or nodules. We describe a case of cutaneous leiomyosarcoma clinically presenting as a skin tag on the thigh of a 50-year-old male. Histological examination of the lesion revealed a dome-shaped tumor with interlacing fascicles of smooth muscle with pleomorphism, cellular atypia and multiple mitoses. Malignant tumors may rarely present as a skin tag, and these are most frequently basal cell carcinomas. We are unaware of previously reported leiomyosarcoma clinically presenting as a skin tag. This case suggests that solitary, wide-based, papilloma-like lesions or skin tags should be submitted for histologic examination to rule out malignancy.

\section{ACTIVATION OF SRG TYROSINE KINASES WITHIN THE SPECTRUM OF KERATINOCYTIC NEOPLASIA.}

Tamu T. Brown, Rosalie Elenitsas, and John T. Seykora University of Pennsylvania Medical School, Department of Dermatology, Philadelphia, PA

The Src-family tyrosine kinases are modulators of growth-regulatory signals within keratinocytes. The relationship between Src kinases and epidermal neoplasia is unexplored in human biopsies. Keratinocytes express 3 Src kinases: Src, Fyn, Yes. Activation of these kinases corresponds to tyrosine phosphorylationÂ near the active site, tyrosine 416 of human Src. This study compared the levels of total Srckinase protein and activated Src kinases within a spectrum of lesions ranging from actinic keratoses (AKs) to squamous cell carcinoma (SCG). An antibody that recognizes an epitope in Src, Fyn, and Yes revealed the total level of Src kinase protein; an antibody specific for the phosphoryated active site tyrosine determined the level of Src kinase activation. The level of Src-kinase expression and activated Src kinases was evaluated in paraffin imbedded sections of AKs, SCG insitu (SCIS), and SCG containing adjacent unremarkable epidermis. The total level of Src kinase protein in lesions was similar to unremarkable epidermis. The level of Src kinase activation was elevated within approximately one-third of AKs and SCIS, but was increased in over $80 \%$ of SCGs. These results demonstrate Src kinases are activated in epidermal neoplasia, and that increased Src kinase activity is associated with the transition from SCIS to SCC.

\section{MYXOID PLEOMORPHIC FIBROMA}

K. Burkert, J. Walsh and J. Keeling

Department of Dermatology, Mayo Clinic Jacksonville, Jacksonville, FL, USA

A 77-year-old white female presented with a two to three year history of an asymptomatic skin-colored papule on the right upper arm. Examination revealed a $7 \mathrm{~mm} \times 7 \mathrm{~mm}$ soft papule, supplanted by a smaller soft papule. Clinical differential diagnosis included an adnexal tumor, neurofibroma, and dermal nevus. Histological examination revealed a sparse proliferation of spindle and stellate-shaped cells within the dermis, including many large cells with bizarre shaped nuclei. Focal multinucleated giant cells were present as well. The background stroma was both collagenous and myxoid, the later being highlighted by diffuse colloidal iron staining. The spindle and stellate-shaped cells demonstrated CD34 and vimentin positivity. Factor XIIIa was focally positive. Actin and S-100 staining were negative. These changes were thought to be consistent with a myxoid pleomorphic fibroma. This is a rare tumor, with only a few reported cases of myxoid variants in the literature to date. The staining pattern of this tumor, along with others in the reported literature, suggest a mesenchymal, fibroblastic origin.

\section{MELANIN PIGMENT UPTAKE WITHIN PAGET CELLS FM Cady, JR McEvoy and JS Metcalf}

Department of Pathology, Medical University of South Carolina, Charleston, SC

Background: The differential diagnosis of intraepidermal neoplasms showing cell clusters and/or single units with upward migration includes Pagets disease, melanoma in-situ, Bowens disease, and Pagetoid reticulosis. Purpose: We describe three cases of Pagets disease of the nipple with melanin pigment uptake by Paget cells. Results: Clinical and pathologic material of three women with biopsy proven Pagets disease of the nipple was reviewed. Clinically, two cases demonstrated an excoriated nipple, one of which also showed a zone of depigmentation. The clinical appearance of the third case was not available. Histologically, each case demonstrated Paget cells singly and in clusters with cytoplasmic uptake of melanin pigment. Melanocytes were found in each case, but were neither increased in number nor showed atypical forms. The dendritic processes of melanocytes were found to encase single Paget cells. Pigment was also found dispersed in the papillary dermis and within melanophages. Conclusions: Melanin pigment uptake by Paget cells represents a significant potential pitfall in ultimately arriving at the correct diagnosis. Misdiagnosing these cases as a melanocytic lesion could potentially delay the management of an underlying breast cancer. Careful examination of melanocyte and melanin distribution with respect to Paget cells is warranted in such cases.

\section{SYRINGOGYSTADENOCARGINOMA PAPILLIFERUM IN SITU: A CASE REPORT AND REVIEW OF THE LITERATURE}

J. Cai, P. Patel, R. Rasberry, A. Slominski and L. Balazs

Department of Pathology and Laboratory Medicine and Division of Dermatology, Department of Medicine, University of Tennessee Health Science Center at Memphis, Memphis, TN, USA

Syringocystadenocarcinoma papilliferum is an exceedingly rare malignant neoplasm of the apocrine glands. There are only about half a dozen cases reported in the literature with one case being an in situ lesion. A 32-year-old Nigerian female presented with a 1-cm, hyperpigmented, slow-growing verrucous nodule located on her midposterior neck. The lesion had been present since birth. Histopathological examination revealed bilocular cystic cavities with papillary projections lined by double layers of epithelium. The luminal layer was composed of columnar cells with decapitation-type secretion. The fibrovascular stroma within the papillary projections contains numerous plasma cells with some lymphoid cells. The cystic cavities showed close apposition to the epidermis of the skin with focal, keratinizing squamous epithelium lining that was contiguous to the infundibular epithelium in foci. Necrosis en masse was present within 
the tumor. There were focal areas of solid aggregates of tumor cells with crowded, pleomorphic, and hyperchromatic nuclei. Many mitoses, some of them atypical, were identified. The tumor was confined within the cystic cavities with no dermal invasion. A diagnosis of syringocystadenocarcinoma papilliferum in situ was made.

\section{CD44 AND HYALURONATE IN THE DIFFERENTIAL DIAGNOSIS OF DERMATOFIBROMA AND DERMATOFIBROSARCOMA PROTUBERANS \\ Emel Calikoglu ${ }^{1}$, Eric Augsburger ${ }^{2}$, Pierre Chavaz ${ }^{2}$, Jean-Hilaire Saurat $^{2}$ and Gürkan Kaya ${ }^{2}$ \\ ${ }^{1}$ Department of Dermatology, Fatih University Medical School, Ankara, Turkey, and \\ ${ }^{2}$ Department of Dermatology, University Hospital of Geneva, Geneva, Switzerland}

The histological distinction between dermatofibroma (DF) and dermatofibrosarcoma protuberans (DFSP) may be difficult. GD34 and Factor XIIIa have been used to differentiate DF from DFSP, but there is a lack of specificity. CD44 is a membrane glycoprotein which has multiple isoforms generated by alternative splicing of variant exons. CD44 is the principal cell surface receptor for hyaluronate (HA). In this study we explored the expression of standard CD44 (CD44s) and its isoforms (CD44v3, CD44v4, CD44v5, CD44v6, CD44v7, CD44v7v8, and CD44v10), and HA in DF and DFSP. Immunohistochemistry was performed on the biopsy specimens of 15 cases of DF and 4 cases of DFSP, using antibodies for the CD44s and its isoforms, and hyaluronate binding protein (HABP). Tumor cells displayed a strong CD44s immunoreactivity in all cases of DF whereas a faint HA positivity was observed in the tumor stroma. DF cells were negative for CD44v3, CD44v4, CD44v6, CD44v7 and CD44v7v8 but they showed a strong reactivity for CD44v5 and CD44v10. CD44s expression was significantly reduced or absent in all DFSP lesions and the tumor stroma displayed a strong staining for HA. Our results indicate that CD44 and HA can be used as additional diagnostic markers to distinguish DF from DFSP.

\section{IMMUNOHISTOCHEMICAL STUDY OF ONCOGENIC AND PROGNOSTIC MARKERS IN SEBACEOUS TUMORS \\ David S. Cassarino ${ }^{1}$, Aaron Auerbach ${ }^{2}$ and Terry Barrett ${ }^{3}$ \\ ${ }^{1}$ Stanford University, Department of Pathology, Palo Alto, CA, \\ ${ }^{2}$ AFIP, Department of GI and Liver Pathology, Washington, DC, and \\ ${ }^{3}$ Johns Hopkins University, Dermatopathology, Baltimore, MD}

Background: Sebaceous tumors are adnexal tumors with usually lowgradebehavior. The distinction between sebaceous adenomas and carcinomas, especially on small biopsies from sensitive sites (i.e., ocular), may bedifficult. We compared the expression of various oncogenic andprognostic markers by immunohistochemistry in order to distinguishbenign from malignant sebaceous tumors.Design: 10 sebaceous adenomas, 10 sebaceous carcinomas, and 5 cases ofsebaceous hyperplasia, were studied with antibodies directed againstKi67 (mib-1), bcl-2, p53, p21, p27, c-erb2 (Her-2/neu), CD117 (c-kit), cyclin D1, MDM2, and CD99 (mic2).Results: We found that sebaceous carcinomas differed from sebaceousadenomas by having increased staining for p53 (50\% vs $11 \%$ ) and mib-1(average 1.8+ vs. $1.0+$ ), and significantly reduced levels of bcl-2 (7\%vs $62 \%)$ and p21 $(16 \%$ vs $34 \%)$.The other markers did not differ significantly, although there was atrend towards more Her-2/neu staining in the carcinomas. No cases showedany c-kit staining.Conclusions: We have shown that several markers, including mib-1, p53, p21, and bcl-2, are expressed at significantly different levels insebaceous carcinomas versus adenomas, and may be of use indiagnostically challenging cases. Whether or not any of these markerswill prove useful in predicting prognosis remains controversial.

MYXOFIBROSARCOMA INITIALLY PRESENTING AS A PLEOMORPHIC HYALINIZING ANGIECTATIC TUMOR (PHAT): IS PHAT A PRECURSOR OR UNIQUE TYPE OF MYXOFIBROSARCOMA?

David S Cassarino, Takako Mitsuhashi and Ronald J Barr Departments of Pathology and Dermatology, University of California, Irvine, USA

Myxofibrosarcoma (MFS) is a common soft tissue sarcoma of adults. We present an unusual case which initially showed features of a pleomorphic hyalinizing angiectatic tumor (PHAT). The lesion was characterized by abundant plump, eosinophilic cells exhibiting striking nuclear atypia, set in a dense, hyalinized stroma with a prominent, angiectatic vasculature. Rare mitotic figures were identified. These findings were felt to be most consistent with the diagnosis of PHAT. Four months after removal, local recurrence was noted, which demonstrated findings of a high grade MFS, including a cellular proliferation of pleomorphic spindle cells set in a prominent myxoid stroma. Multiple mitoses, including atypical ones, were present. In light of these findings, the original specimen was reexamined and the diagnosis was revised to high- grade MFS. MFS, especially low-grade lesions, may be confused with several benign lesions. Previous cases mimicking PHAT have not been reported. We describe a unique case of high-grade MFS which initially showed characteristic features of a PHAT, raising the possibility that MFS may occasionally arise in, or have areas that masquerade as, PHAT. It is possible that PHAT, currently considered a benign neoplasm, may actually represent a peculiar form of MFS of low malignant potential.

\section{ATYPICAL RESPONSE OF XERODERMA PIGMENTOSUM TO 5-FLUOROURACIL: A HISTOPATHOLOGICAL IMAGE ANALYSIS STUDY REVEALS NEW INSIGHT INTO ETIOPATHOGENESIS \\ S.A. Centurion, H. Li, M.J. Kim and W. G. Lambert Departments of Pathology and Dermatology, U.M.D.N.J.-New Jersey Medical School, Newark, NJ, USA}

Xeroderma pigmentosum (XP) is a recessively inherited genodermatosis associated with extreme sun sensitivity, defective repair of several types of sunlight induced adducts in cellular DNA, and numerous, early-onset skin cancers. The dry, rough skin corresponds to progressive cytologic atypia and loss of polarity in the underlying epidermis. Associated with these changes are immune deficiencies against ultraviolet radiation-induced skin cancer. 5-Fluorouracil (5-FU) is a DNA synthesis antimetabolite used against several types of cancers. Applied topically in normal subjects it is associated with moderate to severe inflammation in areas where actinic keratoses have arisen followed by ablation of the actinic keratoses which is dependent on the inflammation. We applied 5-FU to the sun-exposed skin of two patients with $\mathrm{XP}$, a 14 year-old light complected black male and a 14 year-old Caucasian female. No inflammation was observed, but marked improvement in the clinical presentation of the skin was seen, as well as an absence of new malignancies. This change was confirmed histopathologically and correlated with normalization of polarity and cytologic changes in the epidermal cells. These histologic findings were quantitated using computerized image analysis. These results may be due to activation of alternative DNA repair pathways in these nucleotide excision repair deficient cells. 
AGGRESSIVE BASALOID CARGINOMA WITH PILOSEBACEOUS DIFFERENTIATION OF THE HEAD AND NECK: REPORT OF SIX CASES

S. A. Centurion, A. Baisre, N. Mirani, S. Baredes, R. Turbin, P. D. Langer and W. C. Lambert.

Departments of Pathology, Dermatology, Otolaryngology and Ophthalmology, U.M.D.N.J.-New Jersey Medical School, Newark, NJ

We report six cases of a basaloid neoplasm arising on the head and neck showing immature sebaceous gland and focal immature hair follicle differentiation. Four cases arose in the periorbital area, one on the lower lip, and one on the neck. In all six cases extensive involvement of the surrounding soft tissues was observed. In five cases the tumor involved and was particularly aggressive in bone, requiring extensive resection. In two cases tumor extended to and involved the dura mater of the brain. Three cases required enucleation. Patients ranged in age from 30 to 81 years and consisted of four males and two females. No infection, syndrome, or other predisposing factor was known to be present. No patient was known to be a smoker or to engage in any other risk activity for cancer. No evidence of metastasis was noted in any patient. We conclude that the basaloid neoplasm showing immature pilo-sebaceous differentiation of the head and neck is a distinct entity with a much more aggressive course than basal cell carcinoma and a different progression from that of any other adnexal neoplasm, including sebaceous carcinoma.

\section{DIFFERENTIAL DIAGNOSIS OF BULLOUS SKIN LESIONS IN THE PEDIATRIC POPULATION \\ P. Chandra and E. Velazquez \\ Department of Pathology, NYU Medical Center}

We present a case of a male infant born to a 21 year-old mother without significant past medical history. Clinically, he presented with diffuse bullae and thickened patches of yellow to brown skin exhibiting Darier's Sign. This condition affected the infant's entire integument. There was also mild hepatosplenomegaly, detected radiologically. Blood tests and a bone marrow biopsy were normal. The differential diagnosis, clinically, included diffuse cutaneous mastocytosis, bullous genodermatosis, epidermolysis bullosa, epidermolytic hyperkeratosis, incontinentia pigmenti, and staphylococcal scalded skin syndrome. Histologically, there was a proliferation of mast cells within the papillary and reticular dermis, which exhibited positivity with Leder staining, thereby confirming the diagnosis of diffuse cutaneous mastocytosis. Mastocytosis is a condition characterized by mast cell hyperplasia in the bone marrow, liver, spleen, lymph nodes, gastrointestinal tract, and skin. Diffuse cutaneous matocytosis is a rare subset limited to the skin. It occurs in about two percent of cases of mastocytosis and usually affects children before the age of three. This case, although extremely rare, gives an excellent overview of the differential diagnosis of bullous lesions in the pediatric population.

\section{SUBCUTANEOUS NODULES AT INJECTION SITES: HISTOPATHOLOGICAL PITFALLS IN DIAGNOSIS}

I.H. Chaudhry, H. Chong*, K. Brady**, D. Metze*** and E. Calonje Department of Dermatopathology, St John's Institute of Dermatology, St Thomas' Hospital, London. *Department of Cellular Pathology, St George's Hospital, London

Subcutaneous swellings uncommonly occur at vaccination or desensitization injection sites and may present long after the procedure.
Such lesions can show a range of histopathological appearances that are not well recognized, often leading to erroneous diagnoses. We describe the clinicopathological features of 13 cases (12 adults, 1 child) with subcutaneous swellings presenting at injection sites (upper arm $\mathrm{n} 12$, thigh $\mathrm{n} 1$ ). In the cases studied the histological appearances were varied, although predominantly the subcutis was involved. Six cases showed a mixed panniculitis with focal fat necrosis and fibrosis. Four cases demonstrated a pseudolymphomatous pattern, mimicking a $\mathrm{B}$ cell lymphoma. One case was reminiscent of lupus profundus and 3 other cases resembled deep granuloma annulare. Importantly, all cases contained histiocytes with violaceous granular cytoplasm, representing ingested aluminium salts employed for adsorption of antigens in the injection preparations. Energy dispersive x-ray microanalysis in 8 cases confirmed a positive aluminium peak. Electron microscopy in 3 cases showed crystalline material in the histiocytes. In conclusion, the spectrum of histological appearances in so called aluminium granuloma can be misleading and it is important to identify the characteristic histiocytes of this condition, for otherwise erroneous diagnoses, with serious clinical implications, can follow.

\section{NON-NEURAL GRANULAR CELL TUMOUR: A GLINICOPATHOLOGICAL REVIEW OF 11 CASES \\ Dr I.H. Chaudhry and Dr E. Calonje \\ Department of Dermatopathology, St John's Institute of Dermatopathology, St Thomas' Hospital, Lambeth Place Rd, London SE17EH}

Cutaneous granular cell tumour is a well-characterised benign neoplasm of Schwann cell origin. However, there remains a sub-category of neoplasms that appears non-neural in origin and thus requires further appraisal. We present on 11 clinicopathological cases of non-neural granular cell tumour, highlighting the histological patterns, immuno-profile, clinical features and behaviour. The results showed that lesions most commonly occurred in young to middle aged adults ( 9 cases, median $=33$ years, age range: $6-56$ years), with a slight female predominance. The lesions presented as painless nodules, mainly on the extremities or face. Local excision was the treatment of choice and up to date follow-up reveals no local recurrence or metastasis. Histologically, the lesions were polypoid (8 cases) or intra-dermal (3 cases), and composed of elongated spindle-shaped to polygonal cells with prominent granular cell change and focal, variable nuclear atypia. Low level of mitotic activity was noted. Immunohistochemical labelling of the tumour cells showed expression for NKI-C3 (n.11) and CD68, with no staining for S100 protein, SMA, desmin and cytokeratin. In conclusion, this analysis of 11 clinicopathological cases will contribute to the characterisation of this recently described entity, which despite some atypical histological features appears to behave in a completely indolent fashion.

\section{ACUTE MONOCYTIC LEUKAEMIA WITH CUTANEOUS MANIFESTATION}

L. Chen, T. Rodgers, M. Chaffins and K. Maeda

Department of Pathology, Department of Dermatology, Henry Ford Hospital, Detroit, MI, USA

A 50-year-old Caucasian male presented with generalised skin rash. Gingival hyperplasia and hepatosplenomegaly were also noted. His laboratory data showed hemoglobin of $10.3 \mathrm{~g} / \mathrm{dL}$, white blood cell count of $203.6 \mathrm{~K} / \mathrm{uL}$ and platelet count of $72 \mathrm{~K} / \mathrm{uL}$. Peripheral blood smears revealed that $67 \%$ of the white blood cells was monoblasts, $25 \%$ was promonocytes and atypical monocytes. Bone marrow aspirate smears showed that $97 \%$ of the nucleated cells were composed of monoblasts and promonocytes. Approximately $80 \%$ of the immature 
cells showed intense non-specific butyrate esterase activity. The morphologic and cytochemical findings were compatible with a diagnosis of acute monocytic leukaemia. Skin biopsy taken from the left medial thigh revealed a Grenz zone and a dense infiltrate of atypical cells throughout the dermis. The cells were positive for KPl (CD68) immunohistochemical stain. The findings were consistent with cutaneous manifestation of leukaemia (leukaemia cutis). The patient's condition deteriorated rapidly despite chemotherapy and expired 10 days after initial presentation. Leukaemia cutis is more common in acute monoblastic/monocytic leukaemia (AMoL) than in other subtypes of leukaemia. Leukaemia gingival hyperplasia is another characteristic feature of AMoL. Dissemination to the skin is generally associated with a poor prognosis.

\section{ANTITHYROID AUTOANTIBODY-ASSOCIATED DERMATITIS IN INDIVIDUALS WITH UNDIFFERENTIATED CONNECTIVE TISSUE DISEASE - AN UNRECOGNIZED SUBSET OF AUTOIMMUNE DISEASE? \\ W. Cheng ${ }^{+}$, A. Gilliam ${ }^{+}$, and M. Pazirandeh ${ }^{\#}$ \\ Departments of Dermatology ${ }^{+}$and Rheumatology ${ }^{\#}$, Case Western Reserve University/ University Hospitals of Cleveland, Cleveland, Ohio, USA}

Skin conditions in individuals with undifferentiated connective tissue disease (UCTD) are poorly classified and characterised. We reviewed 892 consecutive cases of individuals who had a workup for UCTD. ATAbs were positive in 526(59\%). The $\mathrm{ATAb}(+)$ and $\mathrm{ATAb}(-)$ groups had similar antinuclear antibody (ANA) positivity (32\% versus $28 \%$ respectively), average age (59 versus 58 ), and female-male ratio (8:1 versus $6: 1)$. ATAb positivity was significantly associated with a dermatitis, manifested as erythematous macules/patches or papules on legs, shoulders or upper backs, in 9\% (47/526) individuals versus $2 \%(7 / 366)$ in $\mathrm{ATAb}(-)$ individuals (Pearson's chi-square $=18.7$, $\mathrm{p}<0.0001)$. Sixteen individuals with dermatitis had biopsies, 14 $\operatorname{ATAb}(+)$ and $2 \operatorname{ATAb}(-)$. Routine hematoxylin-eosin and colloidal iron-stained sections were examined. Eleven biopsies (79\%) from $\operatorname{ATAb}(+)$ individuals (5 ANA-negative, 5 with thyroid disease) and one from $\operatorname{ATAb}(-) \operatorname{ANA}(-)$ individuals showed interface/lichenoid dermatitis with mild basal keratinocyte vaculopathy, variable dermal mucin deposition, and perivascular mononuclear inflammatory cell infiltrates in the upper dermis. The interface dermatitis was not significantly associated with ANA positivity or thyroid disease. In summary, we report an ATAb-associated interface dermatitis in UCTD patients which may represent a new subset of autoimmune disease, and suggest that ATAb tests may be a useful marker for UCTD.

\section{MULTICENTRIC RETICULOHISTIOCYTOSIS \\ N. Chiao and J. Tschen \\ University of Texas Houston Medical Center, Houston, TX, USA}

A 38-year-old woman presented with nodules on the face, scalp, ears, hands and elbows. She also developed fevers, chills, fatigue, weakness, myalgia, joint pain, nausea and weight loss of 40 pounds in eight months. Histology revealed large histiocytes with smooth eosinophilic ground glass cytoplasm, consistent with multicentric reticulohistiocytosis. She was treated with methotrexate $25 \mathrm{mg}$ IM per week and prednisone $10 \mathrm{mg}$ PO QD, with relief of her symptoms and improvement in her skin. Multicentric reticulohistiocytosis is a rare histiocytic systemic disease, with facial and hand skin nodules and symmetric arthritis. Pathognomonic signs include "coral beads" and vermicular erythematous papules bordering nostrils. The histologic hallmark for this disease is a nodular infiltrate of histiocytic cells, including multinucleated giant cells containing an eosinophilic, ground glass cytoplasm. Immunohistiochemistry is positive for CD68 and CD3, and negative for B cell markers as well as Langerhans cell markers. Approximately twenty percent of patients with multicentric reticulohistiocytosis have concurrent malignancy, but there is no one predominant type. The disease usually waxes and wanes for many years, with $30 \%$ of patients developing arthritis mutilans. It often regresses approximately seven years after its first appearance.

\section{TATTOO PIGMENT IN SENTINEL LYMPH NODES: A MIMICKER OF METASTATIC MALIGNANT MELANOMA \\ S. Chikkamuniyappa, R. Sjuve-Scott and I.T. Yeh}

Department of Pathology, University of Texas Health Science Center at San Antonio, San Antonio, TX, USA

Tattoo pigment in sentinel lymph nodes in melanoma patients is a clinical challenge. A 77-year-old man presented with a pink nodular lesion of the right upper arm. Biopsy revealed a $1.9 \mathrm{~mm}$ thick, Clark level 4 nodular melanoma. A wide local excision with $2 \mathrm{~cm}$ margins and sentinel lymph node (SLN) mapping was planned. Pre operative lymphoscintigraphy using technetium- $99 \mathrm{~m}$ demonstrated a single strongly pigmented SLN in the right axilla. A prominent tattoo was located on the patient's upper arm in the area of the melanoma. The clinical impression was highly suspicious for metastatic disease and the tissue was sent to pathology for examination. Frozen section showed a lymph node with benign histiocytes. There were black non-polarizable coarse granules in the subcapsular, sinusoidal areas and also in the histiocytes. Review of permanent hematoxylin and eosin sections as well as immunohistochemical analysis with MART-1 and HMB-45 showed no evidence of metastatic melanoma in the SLN. Pigmentation of lymph nodes is not pathognomic of malignant melanoma. Black pigmented lymph nodes can occur by carbon deposits of tattoo pigment as in our case. Histological confirmation of metastatic malignant melanoma in the SLN is imperative before proceeding to complete regional lymph node dissection.

\section{PHOTOPROVOCATION IN THE PATIENTS WITH AN EBV ASSOCIATED HYDROA VACGINIFORME-LIKE ERUPTION \\ KH Cho, K Li, YK Jeon and CW Kim \\ Departments of Dermatology and Pathology, Seoul National University College of Medicine, Seoul, Korea}

There are many reports of patients with a severe hydroa vacciniforme (HV)-like eruption. The cutaneous lesions occur both in sun-exposed and nonexposed areas in these patients unlike a true HV. In most cases, a latent Epstein-Barr virus (EBV) infection has been detected in the skin lesions of these patients. We performed the photo-provocation test in five patients with an EBV associated HV-like eruption. The photoprovocation tests were caried out using the UVA delivered by a SUPUVASUN 3000 light source. A $5 x 5$ sqaure $\mathrm{cm}$ area on the buttock was irradiated three or four times at 24 hour intervals. A skin biopsy was obtained from the lesional skin and photo-provoked lesion in all five patients. $\mathrm{H} \& \mathrm{E}$ staining and immunohistochemical staining were performed. In situ hybridization was also performed to detect mRNA for EBV in the skin biopsy specimens. Skin lesions were induced by repetitive UVA exposure in all five patients. The severity of the phtoprovoked lesion showed correlation with that of the clinical features. The immunohistologic findings of photo-provoked skin lesions were similar to those of original skin lesions. A latent EBV infection was also demonstrated in the photo-provoked lesion. 
CD34+ PIGMENTED FIBROUS PROLIFERATIONS: THE MORPHOLOGIC OVERLAP BETWEEN PIGMENTED DERMATOFIBROMAS AND BEDNAR TUMORS

J. Chu McAllister and U. Sundram

Department of Pathology, Stanford University, Stanford, CA, USA

Pigmented dermatofibrosarcoma protuberans (Bednar tumor) constitute $5-10 \%$ of all cases of dermatofibrosarcoma protuberans, and are usually considered mimics of melanocytic proliferations rather than fibrous lesions. We report two cases of pigmented fibrous proliferations that demonstrate features of both dermatofibromas and DFSP. The first case is a 19-year-old man with a three year history of a slowly growing pigmented lesion on the right arm. On clinical exam the lesion was a $7 \mathrm{~mm}$ firm pigmented papulonodular lesion. The second case is a 31 -year-old woman with a $4-5$ year history of a slowly enlarging, asymptomatic 'dark area' on the right buttock. On clinical exam the lesion is a $2 \mathrm{~cm}$ darkly pigmented flat nodule. Morphologically both lesions are primarily dermal proliferations of spindled cells admixed with pigmented dendritic melanocytes. The lesional cells trap collagen fibers at the periphery and there is basal cell hyperpigmentation. Adnexal structures are effaced but significant trapping of subcutaneous fat is not present. By immunohistochemistry both lesions show focal CD34 positivity but are negative for Factor XIIIa and melanocytic markers. Although overlap between dermatofibromas and DFSP is well documented in the literature, pigmented fibrous lesions with features of both entities are not well described.

\section{PLAQUE-LIKE MYOFIBROBLASTIC TUMOR OF INFANCY}

J. Clarke(Dept. Dermatology), L. Clarke(Dept. Pathology), K. Helm(Depts Dermatology and Pathology), A. Zaenglein(Depts Dermatology and Pediatrics) Penn State Milton S. Hershey Medical Center

We report two unrelated infants that presented for evaluation of large dermal plaques that histologically resembled dermatofibromas. The clinical appearance and histochemical staining, however, support a myofibroblastic origin of the neoplasms. The patients were a 13 month old female with a slowly growing, pink, firm, irregular plaque that was first noticed at 3 months of age, and an 11 month old boy who presented with a pruritic, slowly growing, irregular, nodular plaque that was noticed shortly after birth. MRI revealed no extension of tumor into deeper subcutaneous tissues in either infant. Both infants had normal growth and development. Biopsies of both infants' plaques revealed spindle cell neoplasms dissecting through collagen bundles throughout the entire dermis with overlying psoriasiform epidermal hyplerplasia. The spindle cells were predominantly haphazardly arranged but in a few areas were arranged in small fascicles. Both tumors stained positively for Factor XIIIa and were focally smooth muscle actin positive. Staining for CD34 was negative in both neoplasms. Histologically, both tumors resembled dermatofibromas; however the clinical appearance and positive smooth muscle actin staining best fit with a myofibroblastic neoplasm. The features of this neoplasm and the differential of other spindle cell tumors of infancy will be reviewed.

\section{LOCALIZED CHRONIC FIBROSING VASCULITIS OR LOCALIZED ERYTHEMA ELEVATUM DIUTINUM?}

L. Clarke(Dept. of Pathology), J. Clarke(Dept. of Dermatology), K. Helm(Depts of Dermatology and Pathology)Penn State College of Medicine/MS Hershey Medical Center, Hershey, PA 17033
Localized chronic fibrosing vasculitis is a rare dermatosis that histologically resembles late-stage erythema elevatum diutinum (EED) but has a different clinical presentation. A 62-year-old male presented with bilateral nodules on his heels that first appeared two years ago and over the past six months had become extremely painful. He denied any recent trauma to the sites, and his medical history was significant only for diabetes mellitus, coronary artery disease, and osteoarthritis. Physical exam demonstrated focally ulcerated violet-red three-centimeter nodules on the medial aspects of both heels. Biopsy revealed dense concentric and lamellar fibrosis with foci of leukocytoclastic debris and a sparse infiltrate of histiocytes, neutrophils, eosinophils, and lymphocytes. No granulomas were present, and histochemical stains and tissue cultures for microorganisms were negative. Imaging studies showed no evidence of underlying osteomyelitis, cellulitis, or abscess formation. Laboratory studies demonstrated a markedly elevated IgA level that was shown to be polyclonal on serum immunofixation studies. All peripheral blood cell counts were normal. Thorough evaluations for systemic vasculitides and connective tissue disorders were negative. A diagnosis of localized chronic fibrosing vasculitis was made. This case illustrates the clinicopathologic overlap between this disorder and EED.

\section{BRAF V599E MUTATION IS NOT AGE DEPENDENT: IT IS PRESENT IN COMMON MELANOCYTIC NEVI IN BOTH CHILDREN AND ADULTS \\ J. Cohen, M. Gill, N. Renwick, D. N. Silvers and J. T. Celebi \\ Departments of Dermatology and Pathology, Columbia University}

BRAF encodes a serine-threonine kinase, which acts in the RAS/ RAF/MAPK pathway transducing regulatory signals from RAS to MEK1/2. Somatic mutations in BRAF have been identified in 53$80 \%$ of primary melanomas and $70-90 \%$ of common melanocytic nevi. More than $90 \%$ of these mutations consist of a valine to glutamate substitution at codon 599 (V599E) of exon 15. While a high prevalence of BRAF mutations in common melanocytic nevi has been reported in adults, nevi in children have not been studied. Of interest, we have previously shown that Spitz nevi in children do not harbor mutations in BRAF. To investigate the association of BRAF mutations with patient age, we studied common melanocytic nevi in children for the V599E activating mutation. Tumor cells were microdissected from 6 common melanocytic nevi in children 10 years of age or younger, and analyzed for the V599E mutation in BRAF by allele-specific PCR and gel electrophoresis. In 6 of $6(100 \%)$ nevi, the V599E mutant allele was observed. Our data suggest that similar genetic pathways are involved in the development of common melanocytic nevi in children and adults. The absence of BRAF mutations in Spitz nevi in children is therefore associated with tumor type, not patient age.

\section{PLEXIFORM FIBROHISTIOCYTIC TUMOR: A RARE LOW-GRADE MYOFIBROBLASTIC TUMOR OF CHILDREN AND YOUNG ADULTS}

Nneka I. Comfere ${ }^{1}$ and Iftikhar Ahmed ${ }^{1,2}$ Departments of Dermatology ${ }^{1}$ and Laboratory Medicine and Pathology ${ }^{2}$, Mayo Clinic and Mayo Foundation, Rochester, Minnesota USA.

We describe a 7-year old male with a 7-month history of a plexiform fibrohistiocytic tumor (PFT) presenting as an innocuous $0.5 \times 0.5 \mathrm{~cm}$ nodule on the nasal dorsum. Following diagnostic sampling, Mohs' micrographic surgery was performed to ensure adequate removal and patient continues to do well 1 year post-surgery. PFT is a rare neoplasm described initially by Enzinger and Zhang in 1988. It 
afflicts children and young adults (median age: 14.5 years) and has a strong female predisposition. It typically occurs on the upper extremity. Histologically, the tumor has a bland appearance consisting of a biphasic population of varying numbers of fibroblast and histiocytelike cells. Cellular atypia and pleomorphism are lacking and mitotic activity is low. Immunohistochemically and ultrastructurally, the tumor demonstrates myofibroblastic differentiation. The constituent cells have a diploid DNA content. Unique cytogenetic aberrations have been anecdotally identified. PFT behaves in a locally aggressive fashion and regional nodal disease can occur despite adequate excision. In rare instances, metastasis to the lungs has been described. No association between specific clinicopathologic features and outcome has been identified. In summary, PFT is a rare but distinct low-grade myofibroblastic tumor of children and young adults with a misleading clinical and histological appearance.

\section{GUTANEOUS SOLITARY FIBROUS TUMOR OF UNCERTAIN MALIGNANT POTENTIAL \\ B. Shapiro ${ }^{1}$, J. Cottonl and S. Billings ${ }^{2}$}

Skin Pathology Services, St. Joseph Mercy Hospital, Ann Arbor, MI ${ }^{1}$ and Indiana University School of Medicine, Indianapolis, $\mathrm{IN}^{2}$

Solitary fibrous tumor (SFT) is an uncommon mesenchymal neoplasm that typically arises in the pleural cavity but may also be seen at extra-pleural sites including liver, lung, thymus, orbit, soft tissue, and rarely the skin. The diagnosis of cutaneous SFT is challenging due to its histologic variability and lack of distinctive immunophenotype. We report a case of SFT with unusual histologic and immunophenotypic findings. A 23-year-old man presented with a two-year history of a painful, enlarging scalp mass. Histologic examination revealed a variably cellular neoplasm composed of tumor cells with uniform oval nuclei embedded in a collagenous matrix. In areas there was a prominent hemangiopericytoma-like vasculature. The tumor was strongly immunoreactive for CD34 and had some immunoreactivity for S100. The diagnosis of SFT was rendered based upon the histologic features and strong immunoreactivity for CD34. Immunoreactivity for $\mathrm{S} 100$ is rarely seen in SFT. The tumor had a mitotic rate of $8 / 50 \mathrm{hpf}$. Mitotic rates greater than 5/50 hpf have been associated with aggressive behavior in SFTs of soft tissue. The significance of aggressive histologic attributes with clinical outcome is yet to be determined in cutaneous SFT. Cutaneous SFTs should be considered in the differential diagnosis of superficial spindle cell tumors.

\section{PRIMARY GUTANEOUS LYMPHOBLASTIC LYMPHOMA IN CHILDREN- COMPARISON WITH ITS MEDIASTINAL COMPONENT AND MYELOID SARCOMA \\ Cotton, $\mathrm{J}$ and Hood, AF}

Skin Pathology Services St. Joseph Mercy Hospital, Ann Arbor, MI; Department of Dermatology, Wayne State University, Detroit, MI; and Department of Dermatology, Eastern Virginia Medical School, Norfolk, VA.

Lymphoblastic lymphoma (LL) is the second most common subtype of non-Hodgkin's lymphoma in children. The majority of LL are derived from immature $\mathrm{T}$ cells and present with a mediastinal mass with or without bone marrow involvement. The incidence of subsequent leukemic conversion is high. LL presenting as an isolated tumor in the skin has rarely been described in children. In this study, we reviewed the literature and present four additional cases of childhood primary cutaneous lymphoblastic lymphoma (PCLL). We also contrasted PCLL with mediastinal LL and compared the clinical and prognostic features of PGLL with granulocytic sarcoma (GS). Most tumors occurred in the scalp. Histologically, all cases demonstrated a dense monomorphic dermal infiltrate of intermediate sized cells with scant cytoplasm and inconspicuous nucleoli. The majority of cases were of precursor B phenotype, in contrast to primary mediastinal LL. A number of patients demonstrated concomitant bone marrow involvement at presentation and concurrent or subsequent conversion to acute lympoblastic lymphoma. We believe there are many similarities between PCLL and GS, both in terms of clinical presentation and the association of acute leukemia. Recognition of this uncommon tumor is important so that appropriate clinical workup and therapy may be instituted.

\section{PROLIFERATING HYBRID FOLLICULAR TUMOR E. Craig ${ }^{1}$ and M. Fung ${ }^{1,2}$ \\ Departments of Dermatology ${ }^{1}$ and Pathology ${ }^{2}$, University of California Davis Medical Center, Sacramento, CA, USA}

Epithelial cysts are commonly encountered in clinical practice, most usually as epidermoid (infundibular) cysts, and trichilemmal (pilar) cysts. A multitude of other cystic structures can arise from the pilosebaceous unit, including dermoid cyst, pilonidal cyst, eruptive vellus hair cyst, steatocystoma, pilomatricoma, as well as eccrine and apocrine hydrocystoma. Epidermoid cysts are thought to derive from the follicular infundibulum, and express keratin 10 but not keratin 17 , whereas trichilemmal cysts are believed to derive from the follicular isthmus, and express both keratins 10 and 17. Proliferating follicular cysts generally exhibit trichilemmal keratinization. In addition to the various aforementioned cysts, hybrid cysts have been reported. The majority of hybrid cysts described have been epidermoid-trichilemmal, in which the upper portion of the cyst shows epidermoid keratinization with a sharp transition inferiorly to trichilemmal keratinization. However other hybrid variants have been reported, including epidermoid-pilomatricoma, epidermoid-apocrine hydrocystoma, trichilemmal-pilomatricoma, eruptive vellus hair cyst-steatocystoma, and eruptive vellus hair cyst-trichilemmal cysts. Here we report, to the best of our knowledge, the first case of a proliferating hybrid cyst containing both epidermoid and trichilemmal components, which presented on the scrotum of a 44-year-old man.

\section{EXPRESSION OF KROX-20 IS INCREASED IN PSORIASIS} JL Curry and JA Reed

Departments of Dermatology and Pathology. Baylor College of Medicine, Houston, TX. U.S.A.

Psoriasis is a common and chronic inflammatory skin disease that afflicts $2-3 \%$ of the world population. There is no cure and the precise cause of psoriasis is unknown. Cytokines however, have been established as critical mediators in the immunopathogenesis of psoriasis. EGR-2 is an immediate early serum response gene that codes for Krox-20, a zinc finger transcription factors that is important in resisting TGF-? mediated apoptosis. The aim of this study was to evaluate Krox-20 expression in epidermal keratinocytes in psoriasis where aberrant response to TGF-? signaling has been shown to occur. Formalin-fixed paraffin-embedded tissue $(\mathrm{N}=38)$ including nine psoriatic plaques (PP), nine lichen planus (LP), ten lichen simplex chronicus (LSG), and ten normal skin (NS) were evaluated for expression of Krox-20 by immunohistochemistry. Frequency and relative intensity of nuclear labeling were evaluated. Strong and diffuse nuclear labeling of keratinocytes was observed in all PP and LSG. The frequency of labeling was significantly greater $(\mathrm{p}<0.05)$ in PP and LSC compared to NS. Lichen planus tended toward absent to 
weak nuclear labeling of keratinocytes. The increased frequency of Krox-20 expression in PP and LSC may lead to resistance of TGF-? mediated apoptosis and contribute to epidermal hyperplasia common to these disorders.

\section{TUMOR LYMPHANGIOGENESIS PREDICTS MELANOMA METASTASIS TO SENTINEL LYMPH NODES}

Sam S. Dadras, Bernhard Lange-Asschenfeldt, Alona Muzikansky, Martin C. Mihm and Michael Detmar

Departments of Pathology and Dermatology, Massachusetts General Hospital and Harvard Medical School, Boston, MA

Cutaneous melanoma is a common and frequently lethal melanocytic neoplasm that can quickly metastasize to regional lymph nodes. Currently, prognosis is determined by measuring tumor thickness but more reliable markers for metastatic spread are urgently needed. We investigated whether the extent of tumor lymphangiogenesis can predict melanoma metastasis to sentinel lymph nodes (SLN). We quantified the extent of tumor lymphangiogenesis, as well as other factors, in excised primary tumors and in SLN biopsy samples from 45 patients with primary cutaneous melanoma. Primary melanomas from patients whose tumors had metastasized to the SLN, along with their metastastic foci, contained prominent hotspots of increased lymphatic vessel density, compared to non-metastatic tumors. Multivariate risk analysis revealed that the lymphatic vascular area (LVA) of primary melanomas, an index of tumor lymphangiogenesis, was the most sensitive prognostic marker for SLN metastasis, and was even able to more accurately predict which tumors would become metastatic to SLN than measuring tumor thickness. The extent of tumor lymphangiogenesis is a highly sensitive $(83 \%)$ and specific (89\%) prognostic marker of lymph node metastasis. Assessment of lymphangiogenesis in primary melanomas may be a more effective approach than measuring tumor thickness in selecting patients with early metastatic disease for aggressive therapy.

\section{DEGREASED EXPRESSION OF SRGASM IN ACTINIC KERATOSES AND SQUAMOUS CELL CARCINOMAS Michael J. Dans, Rosalie Elenitsas and John T. Seykora Department of Dermatology, University of Pennsylvania Medical School}

Srcasm, a Src activating and signaling molecule, is a recently discovered substrate of the Src family kinase, Fyn. Although Srcasm can bind downstream signaling molecules, such as Grb2 and the p85 subunit of phosphoinositide 3-kinase, the physiologic role of Srcasm is unknown. Because Fyn is believed to promote keratinocyte differentiation, we hypothesized that Srcasm may also promote keratinocyte differentiation and, thus, antagonize squamous cell carcinoma (SCG) formation. The purpose of this study, therefore, was to determine if Srcasm expression was decreased in SCC's and actinic keratoses (AK). Using immunohistochemisty with a polyclonal affinitypurified anti-Srcasm antibody, we evaluated the expression of Srcasm in parafin imbedded sections of AK, SCG in-situ (SCIS), and SCG from up to 15 different patients each. The expression of Srcasm in these lesions was compared to normal peri-lesional skin of the same biopsy specimen or normal skin from separate biopsies. We found that Srcasm expression was decreased in approximately $50 \%$ of the AK and SCG-IS samples and in over $90 \%$ of the SCG samples. This increased prevalence of Srcasm downregulation from pre-malignant to less differentiated malignant lesions, supports our hypothesis that Srcasm, by functioning downstream of Fyn, may promote keratinocyte differentiation.
THE DIAGNOSTIC CONCORDANCE OF ACTINIC KERATOSIS AND SQUAMOUS CELL CARGINOMA D. A. Davis MD*, J.P. Donahue $\mathrm{MD}^{\dagger}$, J.E. Bost $\mathrm{PhD}^{\wedge}$ and T.D. Horn MD*

*Departments of Dermatology and Pathology, University of Arkansas for Medical Sciences, Central Arkansas Healthcare System, Little Rock, AR.,

${ }^{\dagger}$ Private practice, Birmingham, AL,

${ }^{\wedge}$ Department of Biostatistics, College of Public Health, University of Arkansas for Medical Sciences

Diagnostic concordance of intraepithelial malignancy is generally only fair. Because the diagnosis of actinic keratosis and squamous cell carcinoma is not uniform and because such terms are not consonant with the nomenclature of other human epithelial malignancies, nomenclature revisions have been attempted. One hundred dermatopathologists were solicited to review 15 tissue sections representing a spectrum of varying thickness epidermal malignancy and to choose either actinic keratosis or squamous cell carcinoma as the diagnosis. Among the 77 participating dermatopathologists intraclass correlation was high for what was perceived as actinic keratosis, squamous cell carcinoma and their differentiation. Development of a two-tiered diagnostic system that retains our present diagnostic capabilities, but better fits the pathobiology of superficial epidermal malignancy is suggested.

\section{ANTI-TNF-ALPHA ANTIBODY INDUCED INTERSTITIAL GRANULOMATOUS DERMATITIS}

April Deng, MD, Bahram Sina, MD, David Strobel, MD and

Anthony Gaspari, MD

Department of Dermatology, University of Maryland, Baltimore, MD

With the advance of our knowledge and understanding of the molecular basis of the immune system and inflammatory process, biological modulators, such as anti-TNF-alpha antibodies, are increasingly used in the treatment of immune disorders and hematogenous neoplasm. Side effects of those biological modulators, such as leukocytoclastic vasculitis, lichenoid drug reaction, acute folliculitis, and superficial granuloma annulare, have been reported. Recently, we encountered two patients who developed interstitial granulomatous drug reaction while receiving anti-TNF-alpha antibodies. The patients, a 48 year-old male and a 54 year-old female, both have severe rheumatoid arthritis and responded poorly to conventional therapies, received Remicade (Infliximab). Pruritic erythematous skin eruptions developed on the trunk and extremities one month after starting the therapy. The lesions are multiple irritated papules and plaques; some are targetoid, measuring several centimeters, with raised borders. Skin biopsies showed an interstitial granulomatous infiltrate in the mid- and deep dermis. The infiltrate is composed of lymphocytes, histiocytes, multinucleated giant cells, and eosinophils, palisade around degenerating collagen. Pathological diagnosis was drug-induced interstitial granulomatous dermatitis. Discontinuation of Remicade led to resolution of the skin lesions in both patients. To our knowledge, this is the first report of such a drug eruption caused by anti-TNF-alpha antibodies.

\section{METAPLASTIC BREAST GARCINOMA PRESENTING AS CUTANEOUS SPINDLE CELL CARCINOMA \\ D.P. DeRienzo and R.J. Barr \\ Department of Dermatopathology, University of California, Irvine, CA, USA}

Squamous cell carcinoma is one of the most common primary cutaneous carcinomas, but on rare occasion, metastatic squamous cell 
carcinoma from a distant site or solid organ can present as a cutaneous lesion. Most metastases to the skin present as dermal nodules or involve the dermal lymphatics, but when they are intimately associated with the epidermis, distinguishing the lesion as primary or metastatic may be extremely difficult and usually requires a clinical history or high index of suspicion. A 71-year-old woman presented with a history of breast carcinoma, status post radiation therapy and subsequent mastectomy. She developed multiple eruptive nodules over her mastectomy site, flank and back two years later. Histologically the lesions appeared to be arising from the surface epidermis and consisted of atypical, predominantly spindle cells, some of which streamed off of the basal layer of the epidermis. Following review of the mastectomy specimen material and performing additional immunohistochemical studies, it became clear that the origin of the carcinomas was metastatic from an underlying metaplastic breast carcinoma.

\section{PRIMARY SEZARY SYNDROME COMMONLY} INVOLVES SKIN WITHOUT EPIDERMOTROPISM

A. H. Diwan, V. G. Prieto, M. Herling, M. Duvic and D. Jones Departments of Pathology (AHD, VGP), Hematopathology (DJ, $\mathrm{MH}$ ) and Dermatology (MD), UT MD Anderson Cancer Center

Sezary syndrome (SS) is a T-cell lymphoproliferative disorder involving the blood, skin and lymph nodes associated with erythroderma. Other cutaneous manifestations of SS include palmoplantar keratoderma, ectropion, and alopecia. Two clinical patterns of SS can be defined: "secondary" SS arising from systemic spread of long-standing mycosis fungoides (MF) and "primary" SS with a much shorter prodromal phase characterized by pruritus, xerosis and scaling culminating in erythroderma. Most previous studies examining the histological features of SS have not distinguished between these two different clinical patterns, and have emphasized that the histological features of SS mostly resemble those of typical MF, including epidermotropism and/or band-like superficial dermal lymphoid infiltrates. In this study, we have concentrated on the histological findings of primary SS and correlated them with the clinical and hematologic parameters at the time of skin biopsy. We note that dermal perivascular lymphoid infiltrates predominate in a majority of primary SS cases, raising the differential diagnosis of benign dermatoses, rather than resembling the classical pattern of MF. This impression is further complicated by commonly observed epidermal changes such as spongiosis, acanthosis and parakeratosis that are likely secondary to tumor-associated pruritus and secondary lichenification.

\section{ACTIVATED PROTEIN KINASES AND OVERALL SURVIVAL IN PATIENTS WITH METASTATIC MELANOMA.}

A. H. Diwan, K. Kim, P. Zhang and V. G. Prieto

Departments of Pathology (AHD, VGP, PZ) and Melanoma Medical Oncology (KK), UT MD Anderson Cancer Center, Houston, TX, USA

Protein tyrosine kinases (PTK) are proteins that may play importants roles in survival, proliferation and progression to metastasis in several tumors, including melanoma. There is little information on the expression pattern of the phosphorylated (i.e. activated) forms of such kinases in melanoma and their impact on overall survival (OS). We sought to determine the expression, by immunohistochemistry, of phosphorylated MAP kinase (pMAPK), Akt (pAkt) and C-kit (pCkit) in primary and metastatic melanoma, and their correlation with OS. Fifteen patients with Stage III and Stage IV (metastatic melanoma) disease were randomly selected from the UT MD
Anderson database. Expression was rated by two investigators on intensity (negative, weak, moderate, and strong) and proportion of reactive tumor cells $(0=<5 \%, \quad 1=5-25 \%, \quad 2=>25-75 \%$, $3=>75 \%$ ). Eleven of the 15 tumors (primary and/or metatasis) exhibited pMAPK expression (73\%). Corresponding percentages were $100 \%$ for $\mathrm{pAKT}$ and $<50 \%(7 / 15)$ for pCkit. OS ranged from less than one year to 9 years. However, there was no correlation between OS and expression of any of these markers.

\section{PENICILLAMINE-INDUCED PANNICULITIS WITH LIPOMEMBRANOUS FAT NECROSIS}

K. Donohue, S. Nori, C. Wilkel, V. Falanga and E. Badiavas Department of Dermatology and Skin Surgery Roger Williams Medical Center, Providence, RI, USA

A 43-year-old female with progressive systemic sclerosis developed painful subcutaneous nodules proximally on her extremities which spread distally to her shins. Initially tender, they became red and evolved to painless, red-brown nodules. She had been taking penicillamine for 19 months and was on no other medication or supplements. History and physical exam revealed no evidence of infection, trauma, injection, or significant travel. Incisional biopsy of an acute nodule demonstrated septal panniculitis, with extensive lipomembranous change, and an associated brisk granulomatous infiltrate with multinucleated foreign-body like giant cells. Deeper adipose tissue demonstrated fibrosed thickened septae with an inflammatory cell infiltrate, consistent with scleroderma profundus. Further studies revealed no infectious organisms, or refractile material. The lesions cleared 3 weeks after stopping penicillamine. She declined a re-challenge and has been disease free for a year. Lipomembranous fat changes are identified in about $5 \%$ of subcutaneous inflammatory disorders; most frequently in venous insufficiency, and also with morphea, lupus pannicultis, erythema nodosum, necrobiosis lipoidica and trauma. Panniculitis with lipomembranous fat changes similar to this case has been reported in a patient with systemic sclerosis on norethisterone acetate. This is a rare and interesting case of penicillamine-induced panniculitis.

\section{PRIMARY CUTANEOUS EWING'S SARCOMA}

K.S. Draft and S.D. Billings

Departments of Pathology and Dermatology, Indiana University School of Medicine, Indianapolis, IN, USA

A 57-year-old woman presented with a three-week history of an erythematous nodule on her palm. The clinical diagnosis was pyogenic granuloma. Histology revealed a circumscribed dermal nodule of uniform, primitive round cells with numerous mitotic figures and apoptopic cells. The tumor cells showed strong membranous immunoreactivity for CD99 and nuclear immunoreactivity for Fli-1. The tumor cells were negative for S-100 protein, cytokeratin AE1/3, cytokeratin 20, chromogranin, synaptophysin, and actin. The diagnosis of Ewing's sarcoma was made. CT scans showed no evidence of an osseous or deep soft tissue primary tumor or metastatic disease. The tumor was excised with $1 \mathrm{~cm}$ margins, and the patient received six courses of adjuvant chemotherapy. One year after diagnosis, the patient is alive without evidence of disease. Extraosseous Ewing's sarcoma (EES) rarely presents as a primary skin tumor and should be considered in the differential diagnosis of small round cell tumors involving the skin. It is important to distinguish primary cutaneous EES from secondary involvement of the skin by ES, as primary cutaneous EES has a more indolent course compared to classic EES or osseous ES. Immunohistochemical stains for CD99 and Fli-1 are useful markers to confirm the diagnosis of cutaneous ES. 
ACID-ORCEIN GIEMSA: A MULTIFUNCTIONAL STAIN

B. Drozdowski and D.A. Mehregan

Department of Dermatology, Wayne State University School of Medicine, Detroit, MI, USA

The hematoxylin and eosin $(\mathrm{H} \& \mathrm{E})$ stain is the most common method used worldwide for routine pathologic evaluation of tissue specimens. Evaluation of cutaneous specimens frequently requires differentiation of elastic fibers, melanin, hemosiderin, fungal elements, mast cells and depositions. For this reason, we used acid-orcein and Giemsa (AOG) stain to evaluate various skin disorders to determine the extent of utility of this infrequently reported stain. As expected, AOG stain functioned well as an elastic fiber stain, highlighting loss of elastic fibers in areas of scar and in disorders such as anetoderma. In pigmentary disorders, AOG stains melanin a dark green to greenish-black pigment while hemosiderin remained yellow-brown to light green color. Fungal hyphae in superficial dermatophytosis and leishmania organisms stained a deep blue. Amyloid stained a "sky" blue color in macular, lichenoid and nodular amyloid cases. The granules of mast cells stain purple in cases of urticaria pigmentosa and mastocytomas. Dermal mucin stained metachromatic lavender. Due to the multifunctional nature of the AOG stain, this simple technique can improve dermatopathologic diagnosis while decreasing the number of special stains necessary to have available in the laboratory setting.

MELANOMA IN SITU WITH EPIDERMAL EFFACEMENT: A COMPELLING ADJUNCTIVE FINDING

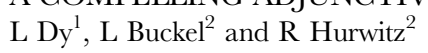

Department of Dermatology, Rush University Medical Center, Chicago, IL, USA ${ }^{1}$ and Dermatopathology Laboratory, Inc. Indianapolis, IN, USA

Since many studies have shown the discordant diagnoses of melanocytic lesions among pathologists despite the existence of an established histologic criteria, we sought to refine the histologic criteria by finding an additional reliable and reproducible objective histopathologic feature, to aid in the diagnosis of melanoma in situ. We performed a retrospective analysis of 100 cases, histologically diagnosed as melanoma in situ and compared to a study control group consisting of junctional benign melanocytic nevi. The epidermis of all the melanocytic lesions were examined for epidermal effacement. Examination of the epidermis in the study group revealed absence of epidermal effacement in only seven $(10 \%)$ cases, whereas ninety-three $(93 \%)$ cases showed absence of rete ridges in some foci, making this an overwhelming majority finding in the cases examined. These results serve as a compelling adjunctive finding that can be used to increase the histologic diagnostic accuracy of melanoma in situ.

EVALUATION OF NUCLEAR OUTLINE WITH FRACTAL MORPHOMETRY TO DIFFERENTIATE SKIN TUMORS GW Elgart, E Welsh and EV Badiavas

Department of Dermatology, University of Miami School of Medicine, Miami, FL, USA and Department of Pathology, Roger Williams Hospital, Providence, RI, USA

Nuclear details are sufficient to differentiate tumors of many types as evidenced by the widespread and successful use of cytology. While cytology examinations are generally optimized to identify the nuclear and morphological characteristics of cell preparations and aspirates, tissue samples are optimized to emphasize the cellular details. Recent studies have validated fractal morphometry for nuclear analysis in cytology. We evaluated the use of nuclear detail analysis employing fractal morphometric analysis in cutaneous tumors to differentiate common and uncommon skin tumors.

\section{AGGRESSIVE SQUAMOUS CELL CARCINOMA IN} KINDLER SYNDROME: AN UNUSUAL CASE

P Emanuel, J Tan, D Rudikoff and R Phelps

Departments of Dermatopathology and Dermatology, Mount Sinai Hospital, New York, NY, USA

A 57-year-old Hispanic man with a personal and family history of bullae and photosensitivity, presented with a fungating, ulcerated Squamous Cell Carcinoma (SCG) on his left hand. Physical exam showed conjunctival injection, urethral stricture, symblepharon, loss of teeth, short stature, nail dystrophy and extensive poikiloderma. Excision and lymph node dissection showed moderate to poorly differentiated deeply invasive SCG, necessitating an amputation. Two of three axillary lymph nodes were positive. A random biopsy of the trunk showed epidermal atrophy, telangiectasia and pigment laden macrophages consistent with poikiloderma. Electron microscopy (EM) illustrated extensive reduplication of the basement membrane with loops, curls and free extensions of the basal lamina in the superficial dermis, reduced numbers of hemidesmosomes and anchoring fibrils, and a basement membrane focally devoid of basal cells. On the basis of the clinical features and the characteristic basement zone changes, a diagnosis of Kindler Syndrome (KS) was rendered. KS is a rare autosomal recessive photosensitivity disorder which contains features of both congenital poikiloderma and dystrophic epidermolytic bullosa, and has unique EM features. Despite the photosensitivity, few cases of malignancy in $\mathrm{KS}$ have been reported.

\section{CD30-POSITIVE PERIPHERAL T-CELL LYMPHOMA}

S. Estrada and W. Anderson

Department of Pathology, St. Joseph's Hospital and Medical Center, Phoenix, AZ, USA

A 47 year-old female presented with a five-month history of left lower extremity cellulitis which was refractory to oral antibiotics. Physical exam revealed focal erythema of the left lower extremity and left inguinal adenopathy. A left leg skin punch biopsy revealed a dense infiltrate present primarily within the deep dermis consisting of markedly atypical large cells with prominent nuclear pleomorphism and focal multinucleation. Many of the cells displayed "horseshoe shaped" or "donut shaped" nuclei, consistent with so-called hallmark cells and/or wreath cells. Scattered mitoses were also observed. Imunohistochemical stains for CD45 and vimentin were positive. Stains for cytokeratins AE1/AE3, GK7, desmin, and S-100 protein were negative. The CD45-positive population was also positive for CD3, CD30, CD45 Ro, and epithelial membrane antigen. They were negative for CD20, CD15, and CD43. A biopsy of the left inguinal lymph nodes revealed involvement by a morphologically similar neoplasm with an identical immunohistochemical staining pattern. Additionally, a CD56 and ALK-1 stain were performed on the lymph node biopsy, and both were negative. This neoplastic process represents a CD30-positive peripheral T-cell lymphoma. The differential diagnosis includes an ALK-1-negative anaplastic large cell lymphoma and a primary cutaneous CD30-positive T-cell lymphoproliferative disorder.

\section{MELKERSSON-ROSENTHAL SYNDROME}

S. Estrada and R. Trepeta

Department of Pathology, St. Joseph's Hospital and Medical Center, Phoenix, AZ, USA 
A 13 year-old boy presented with a six-month history of severe lip swelling. His past medical history was significant for eczema, multiple episodes of urticaria, and two episodes of anaphylaxis. Physical exam revealed severe cheilitis and labial angioedema. The cranial nerves were intact, and there was no evidence of a fissured tongue. A lip biopsy showed noncaseating granulomas throughout the dermis, with extension into the underlying skeletal muscle. Other features included dilated lymphatic channels, some closely approximating the granulomas, and a mild perivascular lymphoplasmacytic infiltrate. The epidermis was acanthotic with parakeratosis and focal acantholysis. Methenamine silver and acid-fast stains were negative. A laboratory work-up revealed an increased total eosinophil count, an increased serum IgE level, and a low complement Ciq level. All other complement levels were normal. The patient also had an unremarkable chest $\mathrm{x}$-ray and a normal angiotensin converting enzyme level. This case represents the monosymptomatic form of Melkersson-Rosenthal syndrome (MRS), also known as cheilitis granulomatosa Meischer. MRS is classically known as a triad of granulomatous cheilitis, facial nerve palsy, and fissured tongue. The onset of granulomatous cheilitis typically precedes the other symptoms, and when present alone, it is known as the monosymptomatic form of MRS.

\section{CUTANEOUS LANGERHANS CELL SARCOMA ASSOCIATED WITH MEDIASTINAL T-CELL ACUTE LYMPHOBLASTIC LYMPHOMA: CLONALLY RELATED NEOPLASMS}

G Eudy, S Soon, B Shehata, K Mann, E Jaffe and A Solomon Departments of Dermatology and Pathology, Emory University School of Medicine, Atlanta, GA, USA, and Laboratory of Pathology, National Cancer Institute, Bethesda, MD, USA

An 8-year-old male presented with several $1-4 \mathrm{~cm}$, indurated, brownish-red nodules with superficial, punctate hemorrhagic crusts localized to the groin, which developed over several weeks. An initial biopsy was consistent with Langerhans cell histiocytosis. A work up, including a chest and abdominal CT scan demonstrated a large mediastinal mass which was diagnosed as precursor $\mathrm{T}$ cell lymphoblastic lymphoma. Treatment with a chemotherapeutic protocol resulted in shrinkage of both the mediastinal and cutaneous lesions. Late in the course of treatment the cutaneous lesions rapidly recurred and spread to various sites. A second skin biopsy showed an extensive diffuse infiltrate of histiocytic-appearing cells extending from the papillary dermis into the superficial subcutis. The cells had markedly pleomorphic, vesicular nuclei and abundant amphophilic cytoplasm. Numerous mitotic figures were present. The neoplastic cells were S-100 protein and CDla positive by immunohistochemistry. The histopathologic diagnosis was Langerhans cell sarcoma. Gene rearrangement studies demonstrated clonal rearrangement of the $\mathrm{T}$ cell receptor gamma gene in specimens from both the mediastinal mass and one of the recurrent cutaneous lesions. The sizes of the PCR products were identical demonstrating a clonal relationship between the two neoplasms.

\section{POST-RADIATION MALIGNANT FIBROUS HISTIOCYTOMA FOLLOWING TREATMENT OF ERUPTIVE SQUAMOUS CELL GARCINOMA IN-SITU IN AN AFRICAN AMERICAN MALE \\ W. Fangman, C. Herman, J. Cook and A. Selim \\ Division of Dermatology and Department of Pathology, Duke \\ University Medical Center, Durham, NC, USA}

A 32-year-old African American male presented with a five year history of multiple verrucous plaques in a Blaschkoid arrangement on the left leg. Biopsies demonstrated squamous cell carcinoma insitu. The patient's medical history did not reveal any heritable or exposure related risk factors. The patient was treated with radiotherapy. Four years later, he presented with a subcutaneous nodule within a prior site of radiation. MRI showed a $2.0 \times 1.2 \mathrm{~cm}$ enhancing nodule in the subcutis. On biopsy, an ill-defined spindle cell proliferation was present in the dermis and subcutis. Marked nuclear pleomorphism and numerous multinucleated cells embedded in a myxoid stroma were noted. Spindle cells stained for CD68. Cytokeratin and S-100 were negative. A diagnosis of Malignant Fibrous Histiocytoma was rendered. Surgical excision was undertaken. A dominant lesion of $\mathrm{MFH}$ was identified, as well as multiple, clinically undetectable foci of early sarcoma. This is an unusual case of multifocal post-radiation sarcoma in an African American patient with squamous cell carcinoma in-situ. While sarcoma is a known risk factor following radiotherapy, the course of this patient would suggest caution in applying radiation in similar clinical settings, as well as long-term clinical follow-up for early detection and treatment of postradiation sarcoma.

\section{INTERSTITIAL GRANULOMATOUS DERMATITIS WITH PLAQUES}

AJ Farmer, LC Wang and MM Medenica

Section of Dermatology, University of Chicago, Chicago, IL, USA

A 74-year-old woman presented with an asymptomatic, 20 by $10 \mathrm{~cm}$, erythematous, indurated plaque on the sacrum with similar lesions in the folds of both legs. Laboratory studies revealed an elevated ANA and ESR but normal rheumatoid factor, TSH, Lyme, anti-DNA, and SSA/SSB antibodies. Biopsies from the sacrum and left leg showed a perivascular and interstitial infiltrate of foamy histiocytes, epitheliod cells, and multinucleated giant cells, as well as lymphocytes and eosinophils in the entire dermis. The patient was diagnosed with interstitial granulomatous dermatitis (IGD) with plaques. The lesions resolved spontaneously. IGD with plaques is a rare but benign disorder of uncertain etiology. Its characteristic histopathology and clinical appearance distinguish it from other granulomatous dermatitis.

\section{ECCRINE POROMA: ONE TUMOR, MANY FACES}

S.B. Forman, F.H. McMullan and F.H. Azmi

Departments of Dermatology and Pathology, Medical College of Virginia, Richmond, VA

Pinkus initially described eccrine poroma as a benign neoplasm of the acrosyringium. The tumor, often consisting of broad anastamosing bands, arises from the epidermis and extends downward into the dermis. However, it may be completely intraepidermal ("hidroacanthoma simplex"), and, at times, appear to be completely dermal ("dermal duct tumor"). The characteristic tumor cell is squamoid, but smaller than the epidermal squamous cell. The cell has a cuboidal appearance with a uniform basophilic nucleus, and intercellular bridges. One can usually observe a sharp line of demarcation between the adjacent epidermis and the tumor cells. The histological sections presented are offered as evidence of the eclectic nature of eccrine poroma. The examples to be discussed include: the intraepidermal variant, the intraepidermal variant with "microcysts, the intraepidermal variant with "macrocysts" the epidermal/classic variant, the dermal variant, the pigmented variant, the irritated variant, the chondroid variant, the syringomatous variant and the focally malignant variant. Each example has the classic features of eccrine poroma and an outstanding individual characteristic. But, all are eccrine poromas. These specimens are from the Medical College of 
Virginia/Francis H. McMullan collection, representing fifty years of practice, teaching and service.

\section{REACTIONS TO PENICILLAMINE: A GASE OF CUTIS LAXA, ELASTOSIS PERFORANS SERPIGINOSA AND "PSEUDO" PSEUDOXANTHOMA \\ S. Frankel, S. Ravis, G. Elgart and R. Kirsner \\ Department of Dermatology, University of Miami, Miami, FL, USA}

This patient was a 61-year-old white female who received several years of penicillamine therapy for the treatment of cystinuria. She subsequently developed penicillamine induced cutis laxa, elastosis perforans serpiginosa, and pseudoxanthoma elasticum like skin lesions. In addition, she suffered from numerous chronic bilateral lower extremity skin ulcerations. Her past medical history was also significant for end stage renal disease requiring hemodialysis and pulmonary fibrosis. She presented to the University of Miami Wound Care Center in 1/04 for treatment of her chronic ulcerations. On physical examination, the patient had multiple large hyperpigmented plaques with central ulcerations on her lower extremities. Some of the ulcers had overlying crust and others were covered with yellow fibrinous tissue. She also had generalized thickened, lax skin with multiple folds. On her neck, thighs, back and arms were violaceous, atrophic, serpiginous plaques with peripheral crusted erosions. A biopsy taken from the patients left thigh revealed dermal elastosis and the features of pseudo-pseudoxanthoma. Two additional biopsies taken from the left thigh demonstrated elastosis perforans serpiginosa. This case highlights multiple skin manifestations of penicillamine therapy.

\section{EPIDERMOTROPIC MUCINOUS ADENOGARCINOMA ARISING AT MUCOCUTANEOUS STOMAL ANASTOMOSIS SITE}

D. Fullen and L. Lowe

Departments of Pathology and Dermatology, University of Michigan Medical Center, Ann Arbor, MI, USA

Polypoid lesions arising at ileostomy sites are uncommon and include inflammatory polyps related to prolapse and neoplastic polyps. Primary mucinous adenocarcinoma arising at an ileostomy site is rare and usually occurs in longstanding ulcerative colitis. An 82-year-old woman presented with an erythematous $1.5 \mathrm{~cm}$ polyp at the mucocutaneous junction of her ileostomy. Her history is significant for a cystectomy with ileal loop 23 years earlier for transitional cell carcinoma of the bladder. She had no prior history of ulcerative colitis. Histologically, a polypoid lesion was produced by variable-sized cystic spaces filled with mucin and lined by columnar epithelium with goblet cells. The epithelial cells demonstrated cytological atypia and occasional mitotic figures were observed. Smaller neoplastic glands infiltrated the dermis and scattered, multiple epidermotropic foci were noted. We present an unusual case of a primary mucinous adenocarcinoma with multiple epidermotropic foci arising at an ileostomy site in a patient without a known history of ulcerative colitis. Primary mucinous adenocarcinoma arising at an ileostomy site is a rare occurrence described primarily in the gastrointestinal literature but could easily be unrecognized by a dermatopathologist. Knowledge of this phenomenon may prove helpful in arriving at the correct diagnosis.

\section{INCIDENTAL ACANTHOLYTIC DYSKERATOSIS AND HAILEY-HAILEY LIKE ACANTHOLYSIS}

E. Gaertner and S. Groo

Department of Pathology, Madigan Army Medical Center, Tacoma, WA, USA
Materials and Methods: 247 consecutive skin specimens covering a three-month period (1/04-3/04) were reviewed to identify incidental foci of Hailey-Hailey $(\mathrm{HH})$ like acantholysis and focal acantholytic dyskeratosis (FAD). Cases in which these patterns comprised the primary process were excluded. All cases were formalin fixed, paraffin embedded, and hematoxylin-eosin stained. Results: Six cases were identified: three shave biopsies (chest, back, face), two excisions (back, face), and one punch biopsy (scalp). This represented $2.4 \%$ of total reviewed. Four specimens had a HH like pattern (1.6\%); two had FAD $(0.8 \%)$. The average patient age was 68 years (range $41-86)$. Four patients were male; two were female. Three cases were associated with epidermal neoplasia: basal cell carcinoma $(\mathrm{HH})$, actinic keratosis $(\mathrm{HH})$, and malignant melanoma in situ $(\mathrm{HH})$. Two were associated with significant inflammation: seborrheic keratosis $(\mathrm{HH})$ and bullous lichen planus (FAD). One biopsy was of lichen simplex chronicus (FAD). The average diameter of acantholysis was $0.3 \mathrm{~mm}$. Four cases showed associated prominent solar elastosis. Discussion: Incidental acantholysis with FAD and $\mathrm{HH}$ like patterns is uncommon. This has previously been postulated to reflect possible preneoplastic epidermal damage. The discussed cases show an association with epidermal neoplasia, solar change, and inflammation.

\section{LYMPHOMATOID PAPULOSIS PRESENTING WITH B- CELL LYMPHOMA: A NEW ASSOCIATION?}

A. Galan, N. P. Wenner and J. M. Henneberry

Department of Pathology, Baystate Medical Center/Tufts University School of Medicine, Springfield, MA, USA

Lymphomatoid papulosis $(\mathrm{Ly} \mathrm{P})$ is a cutaneous T-cell lymphoproliferative disorder, characterised by recurrent crops of necrotic self-healing papules and nodules. Although chronic, LyP typically has a benign clinical course in the majority of cases. Histologically, a malignant appearing T-cell lymphoid infiltrate is seen. The atypical cells often resemble the cerebriform cells of mycosis fungoides or Reed-Sternberg cells in Hodgkin lymphoma. Approximately $10-20 \%$ of the patients go on to develop lymphomas, including mycosis fungoides, CD30-positive anaplastic large cell and Hodgkin lymphoma. We report a case of LyP associated with a B-cell lymphoma. A 50-year-old male, presented with scattered erythematous scaly papules, some with central crust, located on the arms, trunk and leg of one-month duration. A skin biopsy revealed a polymorphous infiltrate with many large atypical lymphocytes, resembling Reed Sternberg cells. By immunohistochemistry, the large cells were positive for T-cell markers and CD30. Subsequently, he developed fever, night sweats and diffuse lymphadenopathy. A lymph node biopsy showed a vaguely nodular proliferation of small to medium lymphocytes. Immunophenotypic and flow cytometric studies best characterised the process as mantle cell lymphoma. Although, LyP has been previously associated with lymphomas of above-mentioned types, this is an extremely unusual case presenting with a low-grade B-cell lymphoma.

\section{DRUG-INDUCED FOLLICULAR LUPUS-LIKE ERUPTION}

A. Galan, D.J. Elpern and H.R. Byers

A 51-year-old female presented with agminate flesh-colored, slightly hyperkeratotic papules, 3-5 $\mathrm{mm}$ in diameter, on her upper back and to a lesser extent on the upper chest, neck and forehead. The papules were centered around the hair follicles and several showed mild perifollicular erythema. Clinical impression was keratosis pilaris with an unusual distribution or follicular mucinosis. Medications included hydrochlorothiazide (HCTZ), begun several months previously, along with potassium supplements. Histologic findings revealed mild 
Abstracts

interface dermatitis with rare individually necrotic keratinocytes and mild pigment incontinence, a superficial and mid perivascular and periappendageal lymphocytic infiltrate with marked dermal mucin deposition and extravasated erythrocytes. No definitive basement membrane thickening was present. Laboratory investigation revealed antinuclear antibodies at 1:640; anti-double stranded DNA and antihistone antibody titers were normal. Following discontinuance of HCTZ and addition of topical steroids, her eruption slowly resolved. Taken together, these findings are those of a new cutaneous manifestation of a drug-induced lupus-like eruption. We have named it druginduced follicular lupus-like eruption to reflect the drug-induced counterpart of the newly described follicular lupus erythematosus.

\section{CNS INVOLVEMENT FROM MYCOSIS FUNGOIDES WITH CD 30 TRANSFORMATION \\ P. Geraminejad, M. Vasef, W. Piette, B. Link and M. Stone Departments of Dermatology and Pathology, University of Iowa Medical Center, Iowa City, IA, USA}

Mycosis fungoides with central nervous system (CNS) involvement is a rare event. Most cases evolve from progressive tumor stage skin disease or CD30 large cell transformation. Cases with CD 30 transformation can show CNS involvement without advanced skin disease. We report a 63 year-old woman with 6 year history of mycosis fungoides with patch and plaque stage disease who developed a single $2.5 \mathrm{~cm}$ tumor on the left chest without lymphadenopathy. Histology revealed a dense infiltrate of small atypical lymphocytes with cerebriform nuclei. Immunohistochemistry showed CD 4+, CD 43+ and CD 30- staining. Within 2 weeks she developed ataxia and right-sided weakness. Magnetic resonance imaging (MRI) revealed left frontal and brainstem masses. Stereotactic biopsy showed a dense infiltrate of atypical lymphocytes with large convoluted nuclei. The immunohistochemistry showed a population of CD 4+, CD 43+, Alk-1- cells with $\mathrm{CD} 30+$ transformation. Gene rearrangement studies for the gamma $\mathrm{T}$ cell receptor on the skin patch and tumor as well as brain tumor failed to reveal a monoclonal population in any of the specimens. No other areas of involvement were found on complete staging. This case is an example of tumor stage MF with CD 30 transformation and CNS involvement.

\section{THE ROLE OF P-75 NGF-R IN THE DIAGNOSIS OF DESMOPLASTIC MELANOMA}

S. Ghosn, A. Radfar, C.M. Stefanato and J. Bhawan

Dermatopathology Section, Department of Dermatology, Boston

University, School of Medicine, Boston, MA, USA

The histologic diagnosis of desmoplastic melanoma is challenging, particularly when the overlying junctional component is absent and when the spindle cells lack melanin pigment. In these instances, the importance of immunohistochemistry cannot be overemphasized. S-100 protein, with a sensitivity approaching $90 \%$, is the primary immunohistochemical stain used for this purpose. HMB-45, although a more specific marker for melanocytes, is often negative in these cases. A marker of schwannian differentiation, p-75 NGF-R has been shown to be a useful confirmatory marker for desmoplastic and neurotropic melanoma, with staining intensity comparable to or better than S-100 protein. We report two cases of desmoplastic melanoma which stained focally and weakly with S-100 protein in comparison with the stronger staining of p-75 NGF-R. In both cases, S-100 staining could have been missed or interpreted as non-confirmatory, thus misguiding the diagnosis. We therefore suggest that p-75 NGF-R be a complementary marker to S-100 protein in differentiating desmoplastic melanoma from other non-neural crest-derived spindle cell tumors.
SPITZOID MELANOMAS IN GHILDREN, LIKE SPITZ NEVI, LACK COMMON ACTIVATING MUTATIONS IN BRAF AND NRAS

M. Gill, J. Cohen, N. Renwick, J. Mones, D. N. Silvers and J. T. Celebi

Departments of Dermatology and Pathology, Columbia University, New York, NY, USA, Ackerman Academy of Dermatopathology, New York, NY, USA

Spitzoid melanoma in children is a rare tumor that can mimic Spitz nevus both clinically and histologically. Recently, dysregulation of the RAS-RAF-MAPK signalling pathway was reported in $89 \%$ of sporadic melanomas due to activating mutations in the BRAF or NRAS gene. BRAF mutations are found in $53-80 \%$ of primary melanomas and $70-90 \%$ of common melanocytic nevi, whereas none are present in Spitz nevi. NRAS mutations occur in $5-37 \%$ of melanomas and 9 $56 \%$ of common melanocytic nevi. The mutational status of NRAS in Spitz nevi is unknown. This is the first study analyzing the mutational profile of BRAF and NRAS in Spitzoid melanomas occurring in prepubertal children (10 years of age or younger). Primary tumor cells were isolated via laser capture microdissection from 10 typical Spitz nevi and 10 metastatic Spitzoid melanomas. Exons 11 and 15 of BRAF and exons 2 and 3 of NRAS were amplified by PCR and directly sequenced. Common activating mutations were not identified in BRAF or NRAS. Absence of the V599E hot-spot mutation in BRAF was further confirmed by allele-specific PCR. We conclude that Spitzoid melanomas in children share clinical, histological and genetic similarities to Spitz nevi, and are genetically distinct from other melanoma subtypes.

\section{NEPHROGENIC FIBROSING DERMOPATHY: A REVIEW OF THE MASSACHUSETTS GENERAL HOSPITAL EXPERIENCE \\ D. Goddard, P. Lio, J. Kay and V. Liu \\ Departments of Dermatopathology, Dermatology and \\ Rheumatology, Massachusetts General Hospital, Boston, MA, USA}

We have undertaken a review of the Massachusetts General Hospital experience of nephrogenic fibrosing dermopathy (NFD). Twelve cases were identified, and the dermatopathologic features of lesional skin biopsies were examined. We looked systematically at nine parameters and graded each on a scale of 0 (minimal or none) to 3 (highest or most): 1) extent of fibrocytic proliferation, 2) extent of collagen thickening, 3) extent of vascularity, 4) extent of pannicular involvement, 5) extent of elastolysis, 6) presence of mucin, 7) presence of calcification, 8) presence of giant cells, and 9) presence of inflammation. Results demonstrate that fibrocytic proliferation, collagen thickening, inflammation, and vascularity most commonly were classified as grade $1-2$; mucin deposition and elastolysis varied from $0-2$; pannicular involvement ranged from 1-3; nearly all had calcification grade of 0 , and there were no giant cells detected in any of the twelve cases. Comparing our results to published reports suggests that there are some interesting observations. Our cases tended to be less fibrocellular, have less mucin, and have more vascularity than the initial 14 cases described by Cowper et al (2001). The implications of the dermatopathologic findings for clinical course and prognosis are currently being evaluated.

BULLOUS MASTOCYTOSIS PRESENTING AS CHRONIC BULLOUS DISEASE OF CHILDHOOD

G. Goldenberg, R. Pichardo, J. Jorizzo and O. Sangueza

Departments of Dermatology and Pathology, Wake Forest University Baptist Medical Center, Winston-Salem, NC, USA 
We report a 6-month old male infant who presented to our pediatric dermatology clinic with a generalized bullous eruption since three months of age. A sepsis work up performed prior to presentation did not reveal any evidence of infection. Clinical presentation revealed multiple tense bullae in a "string of pearls" arrangement and multiple erosions on the bilateral upper extremities and trunk. This clinical presentation was strongly suggestive of chronic bullous disease of childhood. Laboratory evaluation revealed a normal complete blood cell count and glucose-6-phosphate dehydrogenase level. Polymerase chain reaction for herpes simplex virus types I and II was negative. Histological sections demonstrated a large space of separation between the epidermis and dermis which was filled by a monomorphous infiltrate composed of round to oval cells with centrally placed nuclei, consistent with mast cells. Leder and c-kit stains were strongly positive confirming the diagnosis of bullous mastocytosis. Direct immunofluorescence did not reveal IgA deposits or any other findings. Treatment included fluocinonide $0.05 \%$ cream QAM and tacrolimus $0.1 \%$ ointment QHS to active lesions, and silver sulfadiazine $1 \%$ cream to erosions. Improvement was noted during follow up examination.

\section{MINIMAL TELEDERMATOPATHOLOGY: INSTANT IMAGE MESSAGING}

Aldo Gonzalez-Beicos, Liliana Muñoz-Garcia and Aldo González-Serva

Expensive telepathology programs are known for long. Instant messaging, in contrast, is a new trend that will permeate serious enterprises, including dermatopathology. We present an adaptation of microscopy to the MSN Messenger that allows simple and instant sharing of images through the Internet. Any digital camera can act as a webcam, even if no picture is taken. Images through a camera tethered to a microscope can be captured into the PC in real time as video. An inexpensive Belkin VideoBus II card $(\sim 50)$ between the AV cable of the camera and the $\mathrm{PC}$, using an USB port, is used as the capture device (among others). In the MSN Messenger v. 6.1, the Audio/Video Tuning Wizard (from "Tools") is notified that the Belkin capture mode will be used as videocomposite input. The same card could optionally capture audio from any microphone. The above procedure will produce relatively high quality images in the Messenger image subwindow when the digital camera is activated. The interlocutor can broadcast images back. The only limitation is the relatively small size of the Messenger window. This is an example of current mass-appeal technologies that presage the marriage of teledermatopathology and instant messaging.

\section{ULTRASMALL DYSPLASTIC NEVI: ATYPIA CAN BE SEEN IN MOLES LESS THAN 2 MM IN DIAMETER Aldo González-Serva and Liliana Muñoz-Garcia Ameripath New England and Department of Pathology, Boston University, Boston, MA, USA}

The recognized breadth of dysplastic nevi has quickly shrunk. We now present very small dysplastic nevi below any described breadth. A cluster of mostly punched-out nevi derived from a single clinician who recognizes as 'atypical' even very small lesions was also assigned histologic dysplasia on architectural and cytologic basis. From 536 junctional nevi with dysplasia ranging from mild to moderate, 136 $(25.3 \%)$ were $1 \mathrm{~mm}$. in vitro or less while $371(69.2 \%)$ were $1.1-2 \mathrm{~mm}$. Among 155 compound nevi with the same range of dysplasia, 19 $(12.2 \%)$ were $1 \mathrm{~mm}$ or less and $95(61.2 \%)$ were $1.1-2 \mathrm{~mm}$. Moderate or, less so, severe atypia was even seen in more than half of either 18 junctional and 18 compound nevi of dysplastic type between 2.1 and $3 \mathrm{~mm}$. While the above threshold for dysplasia may not be similar to the one than many dermatopathologists could use, it is still evident that sliding down our higher-grade nevi to meet milder degrees of perceived atypia in a more conservative diagnostic scale, the breadth of these nevi still remains minuscule in general. In sum, we adhere to the idea that dysplastic nevi are not only common but can be extremely small: "bonsai nevi."

\section{PUSTULAR DRUG ERUPTION TO VORICONAZOLE}

L. Greene ${ }^{1}$, M. Zavod ${ }^{2}$, P. Krusinski ${ }^{2}$, D. Roseman ${ }^{2}$, S. Hausrath ${ }^{3}$, M. Bosenberg ${ }^{1}$ and D. Cook ${ }^{1}$

Departments of Pathology ${ }^{1}$ and Dermatology ${ }^{2}$,

University of Vermont College of Medicine, Burlington, VT, USA; Department of Medicine ${ }^{3}$,

CVPH, Plattsburgh, NY, USA

A 52-year-old male with Crohn's disease presented with florid pustulosis of the face and trunk. Six months prior to presentation, he received amphotericin for pulmonary aspergillosis. Amphotericin was discontinued after five months due to toxicity, and pharmacotherapy was changed to voriconazole. After four weeks of voriconazole, the acneiform eruption occurred which prompted referral to dermatology. Physical examination revealed innumerable $0.5 \mathrm{~cm}-$ $1.5 \mathrm{~cm}$ pustules, cysts, and inflammatory papules concentrated predominantly over the central face, ears, and conjunctiva, but also involving the chest, shoulders, and back. The clinical differential diagnosis included suppurative folliculitis, papulonecrotic tuberculid, cutaneous Crohn's disease, acne fulminans, and pustular drug eruption. Multiple biopsies demonstrated diffuse neutrophilic and granulomatous dermal inflammation in a predominantly follicular and perifollicular distribution. Microorganisms were not identified by either histology or culture. The histologic differential diagnosis included cutaneous Crohn's disease and pustular drug eruption. Voriconazole was discontinued with gradual clearing of the skin lesions. Three months later, worsening of the pulmonary disease necessitated restarting voriconazole. A recurrent pustular eruption, similar to the original eruption, occurred within weeks and subsequently cleared upon discontinuation of voriconazole. To our knowledge, this represents the first reported case of a voriconazole induced pustular drug eruption.

\section{IMMUNOHISTOCHEMICAL CHARACTERIZATION OF HYALINE GLOBULES IN KAPOSI'S SARCOMA}

N. Gupta, K. Inamdar, M. Chaffins*, C. Ma, A. Ormsby and M. Lee Department of Pathology and Dermatology*, Henry Ford Hospital, Detroit, MI, USA

Hyaline globules (HG) are frequently found in neoplastic cells of Kaposi's sarcoma (KS). Presence of HG, characteristic of but not specific of KS, has been considered to represent erythrophagocytosis. In order to further characterize the nature of $\mathrm{HG}$, we studied 10 cases of cutaneous KS with distinct HG using immunohistochemistry for hemoglobin A, Kappa and Lambda. All KS cases were of nodular type. Also included were 3 cases of glomeruloid hemangioma with similar HG in endothelial cells for the comparision. In selected cases electron microscopic examination was performed. PAS stain with diastase digestion was also done in all cases. Results: HG were diastase resistant and PAS positive. All HG in neoplastic cells of KS and endothelial cells of GH were negative for hemoglobin A. There was variable staining for light chains: focal but strong positivity for kappa, weak and variable staining for Lambda. Some HG was positive for both kappa and lambda. Electron microscopic study demonstrated $\mathrm{HG}$ as homogeneous electron dense inclusions with internal small vacuoles, whorled or granular materials. Conclusions: All HG in neoplastic cells and endothelial cells of GH represent phagolysosomes. Negative stain for hemoglobin A in KS and GH do not support the inclusions being fragmented RBC's. 
THE VALUE OF SENTINEL LYMPH NODE BIOPSY IN THE SETTING OF BORDERLINE MELANOCYTIC PROLIFERATIONS

K Gupta*, K Seilstad*, M Walker**, A. N Crowson***, MC. Mihm Jr**** and C Magro*

Department of Pathology* and the Department of Surgery**, The Ohio State University, Columbus, OH, USA; Regional Medical Laboratory***

St. John Medical Center, Tulsa, OK

The spectrum of melanocytic proliferations ranges from banal to overtly malignant. Borderline melanocytic lesions which bridge these two extremes pose a challenge, as their biological nature remains undefined. We set out to evaluate the utility of the sentinel lymph node biopsy in such lesions. Our compendium was defined by 11 cases of borderline melanocytic proliferations whereby sentinel node sampling was conducted. There were three severely atypical dermal-epidermal melanocytic proliferations manifesting borderline features with nevoid melanoma (calf, shoulder, knee), three arising in association with a deep penetrating nevus (chest, shoulder, and arm), three atypical Spitz's tumors (helix, calf, arm, back), and two atypical pigment-synthesizing melanocytic tumors, resembling equine melanotic disease in one and cellular blue nevus in another (buttock, calf, arm). The patient population comprised seven males and five females ranging in age from age 9-36 (mean: 25 years). At least one positive sentinel lymph node was uncovered in seven of the cases with a positive sentinel lymph observed in all but one case of deep penetrating nevus and atypical Spitz's tumor. The identification of sentinel lymph node positivity in seven of the twelve cases (58\%) validates the role of sentinel lymph node biopsy in the setting of borderline melanocytic proliferations.

\section{ABERRANT GLYCOSYLATION AND ITS CORRELATION WITH EXPRESSION OF GNTV AND MATRIPTASE IN MALIGNANT MELANOMA}

T. Handerson, E. Miyoshi, M. Ueda, N. Taniguchi and J. Pawelek University of Massachusetts Medical School, Worcester, MA, Osaka University, Japan, Kobe University, Japan, Yale School of Medicine, New Haven, GT

Acetylglucosoaminyltransferase V (GNTV), an enzyme responsible for branching of asparagine-linked oligosaccharides is associated with tumor invasion and metastasis. Betal-6 branched oligosaccharides, detected with the lectin, LPHA, are associated with tumor progression in human breast and colon cancer. They were found to be common to 22 types of human cancers, and were particularly expressed in metastases. Matriptase is a proteolytic inducer of both the HGF/cmet and plasminogen activator pathways, highly associated with metastasis. A potential prometastatic effect of GNTV was found to be due to modification of active matriptase by adding betal-6 GlcNAc branching. We investigated expression of LPHA, matriptase and GNTV in pathology specimens of primary invasive and metastatic malignant melanomas. Sequential sections were stained with anti-GnT-V, anti-matriptase, or biotinylated LPHA. Our results showed variable staining patterns, from involving only small regions, to encompassing the entire tumor. The expression of all three markers was closely associated, suggesting that metastatic pathways might have been activated in the staining areas of these tumors. To our knowledge, this is the first report of matriptase expression in malignant melanoma, as well as the first demonstration of an association between matriptase, GNTV and beta 1,6-branched oligosaccharides in vivo.

\section{BIRT-HOGG-DUBE SYNDROME - AN EASILY MISSED DIAGNOSIS}

Valerie Harvey, MD, Anne Rothman, MD, Mark Lowitt, MD,

Bahram Sina, MD and April Deng, MD

Department of Dermatology, University of Maryland, Baltimore, MD

Birt-Hogg-Dube (BHD) syndrome is a rare autosomal genodermatosis characterized by the triad of fibrofolliculomas, trichodyscomas and acrochordons. The gene for BHD was localised to chromosome 17pl1.2 and a germline insertion/deletion was identified to cause premature truncation of the BHD protein, folliculin. Patients carry increased risk for developing lung cysts, spontaneous pneumothorax, and renal carcinoma. Prompt diagnosis is important for patient clinical management. We encountered a 41 year-old white male, otherwise healthy, with classic clinical presentation of BHD, i.e. numerous firm, smooth, whitish papules covering his face and neck, which were developed after adolescence. There is no related family history. Nine biopsies were taken during the past 5 years. The following changes are present: markedly dilated hair follicles, telangiectasia in fibrosing stroma, and melanocytic nevus cells. Diagnoses of epidermal inclusion cyst, fibrous papules, and dermal nevus were given. However, some subtle but diagnostic histopathological features for fibrofolliculoma, perifolliculafibroma, and trichodyscoma were overlooked. Correct diagnosis was reached upon review of all the biopsies. Patient is currently undergoing genetic testing and clinical workups. We present this case to update our knowledge on this rare disease, particularly the diagnostic criteria, differential diagnosis, and diagnostic piffalls, in order to avoid the delay of correct diagnosis.

\section{FOCAL REGRESSION-LIKE CHANGES IN DYSPLASTIC BACK NEVI :A DIAGNOSTIC PITFALL FOR MALIGNANT MELANOMA}

A. Hassanein, N. Depick-Smith, M. Magill and B. Bandarchi Departments of Pathology \& Dermatology, University of Florida College of Medicine, Gainesville, FL, USA

Regression in melanoma can be complete or partial. Melanocytic nevi may show focal regression-like changes (RLC). We studied the incidence of RLC in a total of 777 melanocytic back nevi. These included 17 cases of blue nevi, 28 cases of compound nevi, 385 cases of compound dysplastic nevi, 34 cases of congenital compound nevi, 26 cases of congenital intradermal nevi, 58 cases of intradermal nevi, and 229 cases of junctional dysplastic nevi. The dysplastic nevi were categorised according to the associated cytologic atypia (mild, moderate, and severe). 21 malignant melanomas of the back were also reviewed for regression. RLC were seen with a striking correlation with the degree of cytologic atypia in dysplastic nevi. RLC were seen in $4.5 \%$ of mildly, $9.6 \%$ of moderately, and $17.2 \%$ of severely dysplastic compound nevi. RLC were seen in $10.3 \%$ of mildly, $18.8 \%$ of moderately, and $39.3 \%$ of severely dysplastic junctional nevi. The incidence of regression in non-dysplastic nevi was much less, ranging from $2.9 \%$ to $3.6 \%$. We believe this phenomenon is probably related to trauma/irritation. Caution should be taken before rendering the diagnosis of regressed malignant melanoma on the back since dysplastic nevi may show focal similar changes.

\section{GUTANEOUS ROSAI DORFMAN DISEASE}

\section{R. Higgins ${ }^{1}$, J. Losi-Sasaki ${ }^{2}$ and M. Kinney ${ }^{1}$}

Dept of Pathlogy ${ }^{1}$ and Dermatology ${ }^{2}$,

University of Texas Health Science Center, San Antonio, TX, USA

A 61-year-old female presented with blurry vision. Questioning revealed "Flu-like" symptoms preceding the blurry vision by two 
weeks. An ophthalmologist diagnosed anterior granulomatous dermatitis, which improved with prednisolone drops. One month later, an "itchy rash" and constitutional symptoms including fever, night sweats, and weight loss were reported. Physical examination revealed a viral-type maculopapular eruption over the trunk and proximal extremities. A few weeks later, repeat examination showed progression to a diffuse, reddish-brown, slightly edematous, papular eruption. Lymphadenopathy was not present. Laboratory data revealed a normochromic normocytic anemia, a polyclonal gammopathy, and an elevated ESR. Punch biopsies on two occasions showed a proliferation of mononucleated and multinucleated histiocytic cells with abundant pink cytoplasm. Emperipolesis was easily demonstrated. A background of plasma cells and small lymphocytes were noted. Stains for organisms were negative, and polarizable material was not present. By immunohistochemistry, histiocytes marked with S-100 but were negative for CDla. A diagnosis of cutaneous Rosai Dorfman was made. The cutaneous symptoms improved over six months without medical therapy. Cutaneous Rosai Dorfman disease with constitutional symptoms and laboratory evidence of systemic involvement but without lymphadenopathy has not been previously described. This unusual case suggests that a wider spectrum of disease presentation may exist.

\section{AGGRESSIVE PERIPHERAL T-CELL LYMPHOMA PRESENTING AS CUTANEOUS NODULES WITH A DENSE INFILTRATE OF EOSINOPHILS}

W.A. High, K.R. Houpt, M.P. Hoang, C.J. Cockerell and S.H. Kroft University of Texas Southwestern Medical Center, Dallas, TX, USA

A 65 year-old, well-appearing and asymptomatic man presented with a small non-healing ulcer on the right palm present for 6 weeks. He reported a 15 year history of hand and foot "eczema" treated with topical steroids. Six months earlier his right 4th toe had been amputated for "infection", but additional information was not immediately forthcoming. Punch biopsy from the palm demonstrated superficial ulceration, dermal eosinophils and possible cytoplasmic inclusions within keratinocytes, leading to suspicion of parapox infection. Over the ensuing two months his condition deteriorated markedly, and he developed multiple fungated and ulcerated cutaneous nodules. Incisional biopsy of the nodule on the right palm revealed a dense dermal inflammatory infiltrate with a predominance of eosinophils, and a perivascular infiltrate of medium to large lymphocytes with irregular nuclei and mitoses. These atypical lymphocytes were positive for CD3, but were negative for CD4, CD8, CD30, CD56, TIA-1 and EBER in-situ hybridization. TCR-gamma gene rearrangement revealed a clonal population. Retrospective analysis of the amputated toe demonstrated similar features. He declined conventional treatment, and has been reluctant to pursue follow-up. Our case highlights the fulminant course and dense mixed inflammatory cell infiltrate with eosinophils often present in peripheral T-cell lymphoma that may complicate diagnosis.

\section{APPARENT REACTIVATION OF A FIBROHISTIOCYTIC PROLIFERATION WITH FEATURES OF DERMATOFIBROMA AND DERMATOMYOFIBROMA FOLLOWING SYSTEMIC IMMUNOSUPPRESSION \\ W.A. High, C. Charissi, P.D. Cruz Jr., T. Ehrig and C.J. Cockerell}

A 41 year-old man presented with an atrophic, hyperpigmented plaque on the right lower abdomen present since "birth". He denied any prior activity at the site, and had been told it was a "scar" from a prenatal insult. Six months earlier, he developed idiopathic focal sclerosing glomerulonephritis and was placed on $70 \mathrm{mg}$ prednisone per day. He had not demonstrated evidence of lupus eythematosus. Shortly after beginning this regimen, erythematous and tender papules developed around the quiescent plaque. He had tapered his prednisone dose to $60 \mathrm{mg}$ per day, but additional papules continued to erupt. An ellipse biopsy was performed which included a portion of the atrophic plaque and several surrounding papules. Histological examination revealed a proliferation of fibrohistiocytes between and amongst collagen bundles. In some areas, fibrohistiocytes entrapped collagen in a fashion reminiscent of a dermatofibroma. In other areas, particularly that of the atrophic plaque, the fibrohistiocytes were less numerous and more delicate in appearance. Scattered rudimentary fascicles were demonstrated. Adnexal structures were preserved. Immunohistochemical staining revealed the fibrohistiocytes to be positive for factor XIIIa and actin, but negative for desmin, CD34, S-100, procollagen I, and CD68. This lesion demonstrated unique clinical/histiological aspects not well characterized in the literature.

\section{LIPEDEMATOUS ALOPECIA: COULD IT SOMETIMES BE AN UNUSUAL SEQUELA OF DISCOID LUPUS ERYTHEMATOSUS?}

W.A. High and M.P. Hoang

Departments of Dermatology and Pathology, University of Texas Southwestern Medical Center at Dallas, TX, USA

Lipedematous alopecia is a rare entity of uncertain etiology characterized by a diffusely thickened and boggy scalp, with varying degrees of alopecia. Six of eight cases in the literature have occurred in African American women. Classic histopathologic findings include increased subcutaneous tissue, varying degrees of inflammation and fibrosis, and an absence of significant mucin deposition. Herein, we report an additional case of lipedematous alopecia occurring in an African American woman who also demonstrated clinical, historical, and histopathologic findings reminiscent of discoid lupus erythematosus. Explicit observation of such an occurrence has not been made previously, although hyperkeratosis, follicular plugging, mild perivascular or periadnexal infiltrates, and fibrosing alopecia have been reported in lipedematous scalp, possibly the residua of other cases with associated discoid lupus-like features. For further comparison, we contrast our case with a second patient with lipedematous scalp, which maintained histological similarities to lipedematous alopecia, but was lacked any significant hair loss. It is possible that some cases lipedematous alopecia may represent an unusual sequela of discoid lupus erythematosus. Nevertheless, we cannot exclude the possibility of two diseases occurring independently, nor that early changes of lipedematous alopecia might share histopathological and/or clinical findings with discoid lupus erythematosus.

\section{COMPLETELY REGRESSED PRIMARY CUTANEOUS MALIGNANT MELANOMA WITH NODAL AND/OR VISCERAL METASTASES}

WA High, D Stewart, CRH Wilbers, CJ Cockerell, MP Hoang and JE Fitzpatrick; Departments of Dermatology, University of Texas Southwestern Medical Center, Dallas, TX; University of Colorado Health Sciences Center, Denver, CO, USA

Partial regression of primary cutaneous malignant melanoma is not uncommon, and may predict a higher likelihood of metastasis and decreased survival. Complete histologic regression of a primary cutaneous melanoma is a rarer occurrence of uncertain significance, with only 30 cases reported in the literature. Herein, we detail five additional cases of complete histologic regression of a primary cutaneous melanoma, which were discovered upon presentation with metastatic 
disease. A pigmented lesion, or its remnant, coupled with historical information was strongly suggestive of cutaneous malignant melanoma. However, histologic examination of the lesions in toto using multiple levels, and in some cases immunohistochemical stains, failed to reveal residual melanoma. Our cases are typified by the presence of metastasis of malignant melanoma to regional lymph nodes, with the absence other suspicious lesions or other malignancies. Through these instructive cases, the concept of completely regressed primary cutaneous melanoma is reviewed and the literature critically appraised. When considering a diagnosis of completely regressed primary cutaneous melanoma, it is important that the cases be welldocumented and biopsy-proven. All patients with a diagnosis of metastatic melanoma with an occult primary lesion require a thorough and complete skin examination and consideration of completely regressed cutaneous melanoma within the differential.

\section{CUTANEOUS MANIFESTATIONS OF JUVENILE HYALINE FIBROMATOSIS}

N.J. Hilliard, Y. Hammers, E. Martin, P. Howard, E.M. Omura and D.R. Kelly

Departments of Pathology, Dermatology, and Plastic Surgery, University of Alabama-Birmingham, St. Vincent's Hospital, and Children's Hospital, Birmingham, AL, USA

An 11-year-old adopted male presented with deforming scalp and bilateral posterior auricular nodules, joint contractures, and gingival hypertrophy. Histopathologic examination of the skin nodules revealed a normal epidermis with areas of extracellular eosinophilic ground substance surrounding benign spindle-shaped (myo)fibroblasts in the dermis and subcutis, alternating with hyalinized, acellular zones. The spindle cells did not show cytologic atypia. A diagnosis of juvenile hyaline fibromatosis (JHF) was given. The patient had been seen multiple times in the past for similar recurring, disfiguring dermal and subcutaneous nodules. The patient had a history of osteolytic lesions of the distal radius and ulna and hyaline deposition involving the sigmoid colon and rectum. JHF is a rare autosomal recessive hyalinosis with mutations of the Capillary Morphogenesis Gene-2 (CMG-2) at chromosome 4q21, a region that encodes a binding protein that concatenates capillary collagen IV to laminin. Other genetic abnormalities include mutations in dermal collagen (types I and III). Disease onset occurs early in life. Organ involvement is an ominous sign usually resulting in death during infancy or early childhood. Surgery is the only presently available treatment.

\section{PROMINENT PAPILLARY DERMAL EDEMA IN DERMATOPHYTOSIS}

D. Hoss, A. Berke, J. Grant-Kels, M. Murphy and P. Kerr Department of Dermatology, University of Connecticut Health Center, Farmington, GT, USA

The histological findings in tinea corporis are variable, but include parakeratosis, often with neutrophils, spongiosis, and dermal inflammation. A recent study found some degree of papillary dermal edema in 17 to $23 \%$ of tinea cases. We report five cases of tinea corporis in which prominent papillary dermal edema (PDE) was the salient histological finding. Prominent PDE is often associated with diagnoses such as Sweet syndrome, polymorphous light eruption, allergic contact dermatitis and arthropod bite reactions. Interestingly, the submitted clinical diagnoses for our cases included Sweet syndrome (2), arthropod bite reaction (2), immunobullous disease (2), fixed drug eruption (1), eczema (1) and tinea (2). Our first case was a 25 -year-old female with recurrent edematous plaques in fixed sites. Her lesions completely cleared with repeated courses of systemic steroids, but recurred after steroid taper. One fungal hypha was overlooked on her first biopsy and she was erroneously diagnosed with fixed drug eruption or a blistering disease (immunofluorescence negative). Six months later, repeat biopsy showed PDE, and numerous hyphae were seen on PAS stain. This prompted us to carefully examine PAS-stained sections on subsequent cases with prominent PDE. Prominent papillary dermal edema may be a clue to dermatophyte infection.

\section{ACID-FAST (ZIEHL-NEELSEN) STAINING FACILITATES FOLLICULAR COUNTING IN HORIZONTALLY- SECTIONED SCALP BIOPSIES.}

D. Hoss, A. Berke, M. Murphy, P. Kerr and J. Grant-Kels

Department of Dermatology, University of Connecticut Health Center, Farmington, CT, USA

Transverse sectioning of scalp biopsies was first proposed by Headington in 1984. Horizontal sections allow easy analysis of follicular density, accurate determination of hair shaft diameter, and quantification of the anagen:telogen ratio. The terminal to vellus hair $(\mathrm{T}: \mathrm{V})$ ratio is an important parameter in the differential diagnosis of non-scarring alopecias. A vellus hair is defined as having a crosssectional diameter of $0.03 \mathrm{~mm}$ or less, and a diameter not exceeding the thickness of its root sheath. It is tedious to tally the $\mathrm{T}: \mathrm{V}$ ratio in a standard $4 \mathrm{~mm}$ punch biopsy because the entire biopsy can only be visualised using the $2 \mathrm{x}$ and $4 \mathrm{x}$ objectives. It is difficult to identify vellus hairs with this magnification, and one must switch between high and low power, often losing track of which hairs have already been counted. All hair shafts are strongly acid-fast and stain bright red, making it easy to count terminal and vellus hairs with $2 \mathrm{x}$ and $4 \mathrm{x}$ magnification. We show acid-fast (Ziehl-Zeelsen) stained slides from various types of non-scarring alopecias cut at the level of the infundibula, and contrast these to hematoxylin and eosin stained slides at the same level, to demonstrate how acid-fast staining greatly facilitates follicular counting.

\section{DISPLACEMENT OF DERMAL SOLAR ELASTOSIS IN MALIGNANT MELANOMA}

M. L. Howard, T. N. Evans and M. G. Horenstein

Department of Pathology, University of South Alabama, Mobile, AL, USA

Background: Sun exposure plays an important role in the pathogenesis of melanoma. Melanocytic lesions occur in sun-exposed skin and protected skin. Here we study the histologic interaction of benign and malignant melanocytic lesions and solar elastosis (SE). Design: We selected 15 invasive malignant melanomas $(\mathrm{MM})$ and 6 benign melanocytic nevi (MN) occurring in sun exposed areas in patients with significant SE. We measured SE thickness in perilesional skin and SE displacement in lesional skin. Results: SE thickness was $0.64+/$ $-0.11 \mathrm{~mm}$ in $\mathrm{MM}$, and $0.38+/-0.06$ in $\mathrm{MN}(\mathrm{P}<0.05)$. SE displacement was $1.02+/-0.85 \mathrm{~mm}$ in $\mathrm{MM}$, and $0.44+/-0.30$ in $\mathrm{MN}(\mathrm{P}<0.05)$. Tumor cells and host response were responsible for displacement of SE in MM. Thickness of displaced SE was decreased in $6 \mathrm{MM}$ and $1 \mathrm{MN}$ (attenuation), unchanged in $7 \mathrm{MM}$ and $4 \mathrm{MN}$ and increased in $2 \mathrm{MM}$ and $1 \mathrm{MN}$. Conclusion: Displacement of SE may be helpful in the diagnosis of MM. In MM the newly formed tumor and associated host response appear to push down the preexisting solar elastosis. Conversely, long standing benign $\mathrm{MN}$ in sun exposed areas appear to coexist with the SE without significant displacement. 
DESMOPLASTIC SPITZ NEVI

A. Hughes and D. R. Mehregan

Department of Dermatology, Wayne State University, Detroit, MI and Pinkus Dermatopathology Laboratory, Monroe, MI, USA

We have reviewed sixteen patients with the diagnosis of desmoplastic Spitz nevus. The age of our patients ranged between seven years and fifty-four years. We noted a predominance of twelve female patients and four male patients. Histologically, ten of the sixteen cases were well circumscribed and symmetric. In six cases we were unable to ascertain the overall architectural features because of the biopsy technique. Only three cases showed significant junctional melanocytic nesting. In five cases there were rare nests of melanocytes at the dermo-epidermal junction and in eight cases there was no evidence of melanocytic nesting at the dermo-epidermal junction. Dermal mitotic figures were rare, with five cases showing a single dermal mitotic figure. The lesions did not arise in sun damaged skin as evidenced by the rarity of actinic elastosis within our specimens. Additional clinical and histologic findings and follow up will be reported.

\section{SUBCUTANEOUS PHAEOHYPHOMYCOSIS CAUSED BY CLADOPHIALOPHORA BANTIANA}

S. Hussey, R. Gander, P. Southern and M. Hoang

Department of Pathology, University of Texas Southwestern Medical Center, Dallas, TX, USA

Primary subcutaneous phaeohyphomycosis can rarely be caused by Cladophialophora bantiana. Only two cases have been reported and we present the histologic and culture findings of another. A 32-yearold black female with systemic lupus erythematosus presented with a two-year history of multiple, recurrent, tender, ulcerated skin nodules with purulent drainage involving an area measuring approximately $27.0 \times 15.0 \mathrm{~cm}$ on her upper back. She had been involved in a tornado at age 10 and had sustained traumatic implantation of wood splinters in this area. Sections of the excision showed scattered dark pinpoint foci with surrounding suppuration extending to the deep aspect of the specimen. Histology demonstrated features of phaeohyphomycosis - foci of granulomatous and acute inflammation with central clusters of dematiaceous fungal hyphae within a fibrotic dermis and subcutaneous tissue. Chains of brown-pigmented septate hyphae and unicellular conidia were identified. Culture revealed pigmented septate, branched hyphae and unbranched acropetal chains of conidia with no attachment hila. Dark gray, velvety colonies formed in one week on a potato dextrose agar plate incubated at 25 degrees Celsius. These culture features were characteristic of Cladophialophora bantiana. Despite surgical excision and itraconazole therapy, her skin lesions persisted for the next two years necessitating a re-excision

\section{EXPRESSION OF P63 IN PRIMARY CUTANEOUS ADNEXAL NEOPLASMS AND ADENOCARCINOMA METASTATIC TO THE SKIN}

Doina Ivan, M.D., A. Hafeez Diwan, M.D., PhD. and Victor G. Prieto, M.D., PhD

Departments of Pathology and Dermatology, University of Texas M. D. Anderson Cancer Center, Houston

p63, a recently identified homologue of p53 gene, has been reported to be essential in the development of epithelia and is mainly expressed by basal and myoepithelial cells. The purpose of this study was to investigate the pattern of p63 expression in cutaneous adnexal neoplasms and to assess its possible value in the differential diagnosis of primary cutaneous neoplasms versus adenocarcinomas metastatic to the skin. Immunohistochemical analysis using p63 monoclonal antibody (clone 4A4) was performed on formalin-fixed, paraffinembedded archival tissue from 20 benign adnexal tumors, 10 malignant adnexal tumors, and 14 adenocarcinomas metastatic to the skin. The expression of p63 was evaluated in epidermal cells, skin appendages and metastatic tumor cells. p63 was consistently expressed in the basal/suprabasal cells of epidermis and cutaneous appendages, including the basal/myoepithelial cells of sweat glands. Thirteen out of $20(65 \%)$ benign adnexal tumors showed strong p63 expression; the remaining $7(35 \%)$ cases had over $50 \%$ p63-positive nuclei. All primary cutaneous carcinomas, including adenocarcinomas, expressed p63. In contrast, none of the metastatic adenocarcinomas to the skin was positive for $\mathrm{p} 63(\mathrm{p}<0.0001)$. Based on our findings, analysis of p63 expression may be a helpful tool in the differential diagnosis of primary versus metastatic cutaneous adenocarcinomas.

\section{HAIR MATRIX DIFFERENTIATION IN A TRICHOGENIC ADNEXAL NEOPLASM.}

O.H. Iwenofu, W.L. Lamar, S.H. Clark and A.N. Crowson

Departments of Pathology and Dermatology University of Oklahoma Health Sciences Center \& Regional Medical Laboratories, St. John Medical Center, Tulsa, Oklahoma, USA

Pilomatricoma is a distinctive tumor characterized by a dual population of proliferating germinative cells and shadow cells and is beleived to derive from the hair matrix. Rarely, benign and malignant adnexal tumors can show pilomaticoma-like or hair matrix differentiation. We present a 69-year-old man with a ruptured cystic nodule of the left cheek. Histology revealed a dermal tumor composed of nests of basaloid cells which manifested peripheral palisading and abortive hair papilla formation with a modified stroma and pilomatricoma-like areas. This lesion shares features of a trichogenic adnexal neoplasm with those of a tumor having hair matrical differentiation, and has been rarely reported under the designation "follicular" basal cell carcinoma (BCG). This case illustrates an example of this unusual occurrence of hair matrix differentiation in a benign basaloid adnexal neoplasm as characterized by the presence of basaloid and shadow cells; the absence of individual cell necrosis and mitotic activity prompts us to doubt that it represents a form of nodular BCC, but rather it more likely reflects a hamartoma of the follicular anlage.

\section{A CASE OF PSEUDORHEUMATOID NODULE ARISING IN A PREVIOUS SITE OF SQUAMOUS CELL CARCINOMA.}

O.H. Iwenofu, D. Adelson and A.N. Crowson

Departments of Pathology and Dermatology University of Oklahoma Health Sciences Center \& Regional Medical Laboratories, St. John Medical Center, Tulsa, Oklahoma, USA

The pseudorheumatoid nodule (PRN) is a subcutaneous palisading granuloma characterized by a pattern of interstitial histiocytes surrounding an area of collagen necrobiosis and typically unassociated with rheumatoid arthritis. It is part of the spectrum of palisading granulomatous dermatitidis which includes the rheumatoid nodule, the rheumatic fever nodule, the spirochaete-associated juxtarticular nodes of Lyme disease, granuloma annulare, necrobiosis lipoidica and the palisading granuloma of Churg and Strauss. PRN has previously been associated with surgical trauma and with retained suture material at old surgical sites. We present a case of PRN arising in a previous biopsy site of squamous cell carcinoma (SCC). A 48-year-old woman with no history of rheumatoid arthritis or a granulomatous 
diathesis underwent biopsy of a keratotic left forearm skin lesion on 10/14/03 that showed invasive SCG. She had a re-excision of the the same site on $11 / 22 / 03$ that grossly showed a central scar. Histology revealed a scar at the site of a recent biopsy with no residual SCG, in the base of which was an area of necrosis palisaded by histiocytes. No residual suture or other polarizable foreign material was identified. Pancytokeratin and Epithelial Membrane Antigen immunostains were negative. The morphology is considered prototypic for PRN.

\section{BENIGN GUTANEOUS MIXED TUMOR WITH COMBINED FOLLICULOSEBACEOUS AND ECGRINE DIFFERENTIATION}

O.H. Iwenofu, W.L. Lamar, S.H. Clark and A.N. Crowson Departments of Pathology and Dermatology University of Oklahoma Health Sciences Center \& Regional Medical Laboratories, St. John Medical Center, Tulsa, OK, USA

Cutaneous adnexal tumors manifesting divergent differentiation are a rare but recognized subset of adnexal neoplasms. We present a 42year-old woman with a cystic tumor of the skin above the left eyebrow. A biopsy revealed a tumor comprising eccrine tubules lined by a single row of columnar epithelium surrounded by a peripheral small cuboidal layer and containing, adhered to the internal cuticle, mucinlike material. In some areas, the eccrine tubules were associated with sheets of small plasmacytoid myoepithelial cells embedded in a chondroid matrix. Elsewhere, there were cysts containing central keratinous debris associated with a peripheral basaloid epithelial proliferation showing the formation of buds mimicking abortive hair follicle structures. No polarizable terminal or vellus hairs were seen. These two elements were geographically separate in the biopsy but collided in the center of the tissue element. A diagnosis of benign cutaneous mixed tumor with combined folliculosebaceous and eccrine differentiation was rendered. Such tumors present a diagnostic challenge and are frequently confused with neoplasms including the benign mixed tumor (chondroid syringoma) and a variety of eccrine and folliculosebaceous neoplasms and harmatomata. This case illustrates the potential multilineage differentiation of pluripoential germinative cells of the cutaneous adnexae.

\section{ANDROGEN REGEPTOR EXPRESSION HELPS TO DIFFERENTIATE BCG FROM TRICHOBLASTOMA AND TRICHOEPITHELIOMA}

L. Izikson ${ }^{1,2}, \mathrm{~A} . \mathrm{Bhan}^{3}, \mathrm{~A}$. Zembowicz ${ }^{2}$

Department of Dermatology, University of Pittsburgh ${ }^{1}$, and

Department of Pathology, Dermatopathology Unit

${ }^{2}$ and Immunopathology Unit and

${ }^{3}$ Massachusetts General Hospital and Harvard Medical School, Boston, MA, USA

Histological differentiation between basal cell carcinoma and benign trichoblastic neoplasms such as trichoepithelioma and trichoblastoma can be difficult on small biopsies. Therefore, several attempts have been made to identify immunohistochemical differences between these entities. Recent studies showed androgen receptor expression in a number of mature epithelial structures in the skin and in epithelial neoplasms including basal cell carcinoma. In contrast, androgen receptor expression was absent in mature hair follicles or the few trichogenic neoplasms studied to date. These findings suggested that androgen receptor expression might be a useful adjunct in the histological differential diagnosis between basal cell carcinoma and benign trichoblastic neoplasms. Therefore, we performed immunohistochemical analysis of androgen receptor expression in 32 basal cell carcinomas and 10 benign trichoblastic tumors ( 6 trichoepitheliomas and 4 trichoblastomas). In our study, expression of androgen receptor was detected in $78 \%$ of basal cell carcinomas. None of the trichoblastic tumors showed any androgen receptor immunoreactivity. These results confirm the lack of androgen receptor expression in benign trichoblastic neoplasms and indicate that finding of androgen receptor expression points to basal cell carcinoma as the most likely diagnosis.

\section{ACTIVATION OF P44/42 MAP KINASES WITHIN HUMAN EPIDERMAL NEOPLASIA.}

Bryon Jackson, Rosalie Elenitsas and John. T Seykora Department of Dermatology, University of Pennsylvania Medical School

Squamous cell carcinoma (SCG) arises from a series of genetic changes that form a clone of keratinocytes with enhanced growth characteristics. The p44/42 Map kinase pathway is a highly conserved growth regulatory pathway that helps relay critical signals from the cell membrane to the nucleus. Evidence demonstrating activation of the p44/42 pathway in the human cutaneous SCG has not been established. This study examined if p44/42 MAP kinase is activated in lesions of keratinocytic neoplasia. Lesions from the defined stages of keratinocytic neoplasia, normal skin, actinic keratoses, squamous cell carcinoma in situ, and squamous cell carcinoma, were randomly selected from archived material and studied. Antibodies that detect human p44/42 (phosphorylated and unphosphorylated) and only phosphorylated, activated, human p44/42 were used. The intensity and prevalence of cytoplasmic and nuclear staining was evaluated in the lesional cells. The results suggest that there is a not a simple linear relationship between the amount of nuclear staining and the type of lesion. The results show that there was a significant increase in the level of nuclear phosphorylated p44/ 42 staining progressing from an actinic keratoses to a sqaumous cell carcinoma in situ. These findings suggest that p44/42 MAP kinases are activated in keratinocytic neoplasia.

\section{BILATERAL SYSTEMATIZED POROKERATOTIC ECGRINE OSTIAL AND DERMAL DUCT NEVUS WITH UNILATERAL BREAST HYPOPLASIA \\ A. Jayaraman ${ }^{1,2}$, L. Robinson-Bostom ${ }^{2}$, C. McDonald ${ }^{2}$ Department of Pathology, Stanford Medical Center, Stanford and $\mathrm{CA}^{1}$ and Department of Dermatology, Brown Medical School, Providence, RI, USA ${ }^{2}$}

Porokeratotic eccrine ostial and dermal duct nevus (PEODDN) is a rare hamartomatous malformation of the eccrine duct which typically presents at birth or early in life. Multiple punctate pits and verrucous papules and plaques with nonfollicular hyperkeratotic spines are seen over the palms and soles and may extend to the distal extremities. A linear distribution may be present. A wide range of involvement has been reported in the literature, including bilateral systematized involvement. No systemic involvement has been linked to PEODDN to date. Histologic findings are characteristic and include a dilated eccrine acrosyringium with an overlying parakeratotic column or cornoid lamella. There is slight dyskeratosis and loss of the granular layer under the cornoid lamella. Here, we present a case of bilateral systematized PEODDN in an otherwise healthy, 18 year-old Hispanic female. Physical examination is notable for marked hypoplasia of the left breast with overlying verrucous papules and plaques. Multiple punch biopsies were performed and showed findings diagnostic of PEODDN. To our knowledge, this is the most extensive involvement of PEODDN reported in the literature to date and the only case with associated hypoplasia of the breast. 
S-100 PROTEIN POSITIVITY IN MERKEL CELL CARCINOMA: AN UNCOMMON FINDING

S. Jensen and C.M. Stefanato

Department of Dermatopathology, Boston University School of

Medicine, Boston, MA, USA

Merkel cell carcinoma (MCG) is a small round cell tumor which does not characteristically stain with S-100 protein. We report a case of an 83 year-old male with a two-month history of an ulcerated nodule on the right arm. Histopathology revealed small blue round cells arrayed in a trabecular pattern and in diffuse sheets, with scattered mitoses and necrosis. The cells were negative for vimentin, positive for NSE, focally positive for high molecular weight cytokeratins and diffusely positive for low molecular weight cytokeratins, including CK20. The findings were diagnostic of MCC, however, foci of S-100 protein positive cells were also present in the tumor. Upon reviewing cases from Skin Pathology Laboratory at BUSM received between 1/1/ 2000 to $7 / 30 / 2004$, we documented a total of 34 cases diagnosed as MCC; 24 were stained with S-100 protein and this case was the only one that stained positively. S-100 protein positivity of MCG, while having been previously reported, is an uncommon finding. We report our case to stress the importance of morphological and immunoperioxidase features for the diagnosis of Merkel cell carcinoma, particularly in the exceptional cases which exhibit S-100 protein positivity.

\section{ECCRINE MUCINOUS METAPLASIA ASSOCIATED WITH AN APOCRINE GYSTADENOMA}

Jiang $\mathrm{J},{ }^{1}$ Petronic-Rosic V ${ }^{1}$ Hoag $\mathrm{J},{ }^{2}$ and Shea, $\mathrm{CR}^{1}$

${ }^{1}$ Section of Dermatology, the University of Chicago Medical Center, Chicago, IL, USA

${ }^{2}$ Dermatology, Fahey Medical Center, Desplaines, IL, USA

Mucinous metaplasia occurs uncommonly in cutaneous pathology, usually at specialized anatomic locations (genitalia, palms, soles) and within restricted pathologic contexts (inflammation, trauma). Here we report a unique case of eccrine mucinous metaplasia associated with an apocrine cystadenoma. A 13-year-old girl had an asymptomatic, 4-mm nodule on the chest. Histopathology demonstrated a typical apocrine cystadenoma in the upper and middle dermis. Adjacent to this neoplasm was a cluster of coiled eccrine secretory ducts, of which the inner layer was almost entirely replaced by benign-appearing cells containing abundant, non-sulfated acid mucopolysaccharides. At eight months' follow-up, there was no recurrence. Our case demonstrates that, very uncommonly, mucinous metaplasia may be associated with a pathogenetically separate, adjacent neoplasm, in this instance an apocrine cystadenoma.

\section{CHRONOLOGY OF CELLULAR BLUE NEVUS}

L. Jin, E. Arai, T. Tsuchida, F. Ogawa, T. Mitsuhashi, S. Ban, Y. Shimizu, T. Hirose and M. Shimizu

Departments of Pathology and Dermatology, Saitama Medical School, Saitama, Japan

Cellular blue nevus is composed of dendritic melanocytes, together with islands of epithelioid and plump spindle cells. We examined 58 cases of blue nevi. Fourteen cases were common blue nevi. The remaining 44 blue nevi were divided into three types according to its silhouettes. These were nodular (type 1, 18 cases), nodular with notch (type 2, 16 cases), and rounded pushing patterns (type 3, 10 cases). Well circumscribed top heavy nodule was observed in type 1 , and it was limited to the dermis. Type 2 showed budding appearance into the subcutaneous tissue. Type 3 revealed bottom heavy with downward pushing margin. Both type 2 and type 3 were demonstrated biphasic patterns of epithelioid and spindle cells. Perineural distribution of melanocytes was also observed in type 2 and 3 . The size of these types was $3.3 \mathrm{~mm}$ in type $1,4.7 \mathrm{~mm}$ in type 2 , and $7.4 \mathrm{~mm}$ in type 3 . The thickness of these types was $1.6 \mathrm{~mm}$ in type $1,2.5 \mathrm{~mm}$ in type 2 , and $5.2 \mathrm{~mm}$ in type 3 . Our results suggest that cellular blue nevi may be divided into three stages. Type 1 is its early stage, and may be misinterpreted as common blue nevi.

\section{SQUAMOUS ISLANDS IN CLEAR GELL HIDRADENOMA:} A POTENTIAL DIAGNOSTIC PITFALL

A. Kamath*, A. Radfar** and C.M. Stefanato**

*Department of Pathology, Tufts-New England Medical Center, Boston, MA and

**Dermatopathology Section, Department of Dermatology, Boston University School of Medicine, Boston, MA, USA

Clear cell hidradenoma is a benign eccrine tumor with nodularcystic architecture, ductal differentiation and poroid cytology. Squamous islands occurring in eccrine neoplasms are classified into three types, according to their location, histologic characteristics and degrees of cellular atypia. Type I squamous islands resemble normal epidermis and are present in the superficial to middle portion of eccrine poromas. Type II squamous islands are localised in the deeper portion of eccrine poromas and show lumen formation suggestive of acrosyringeal differentiation. Type III squamous islands show atypical features and are limited to malignant eccrine tumors. A 55-year-old man was biopsied for a dermal nodule on his left arm. The initial shave biopsy revealed the surface of an intradermal cystic nodule with numerous whorls and horn pearls of squamatization, reminiscent Âof a well-differentiated squamous cell carcinoma; however, no cytologic atypia, necrosis or mitotic activity were identified; focal clear cell and ductal differentiation were also present. Complete excision revealed clear cell hidradenoma with extensive mixed type I and type II squamous islands. The presence of whorls and pearls of squamoid cells is a rare finding in clear cell hidradenoma that, moreover, in the setting of a superficial biopsy, may be a diagnostic pitfall.

\section{A CASE OF MALIGNANT MELANOMA WITH MULTIPLE PRIMARY LESIONS}

M. Kato, M. Taguchi, A. Kuramochi, T. Suzuki and T. Tsuchida

A 49-year-old Japanese male first visited our clinic at May 13th, 2003. A bean-sized black macule had been noticed in the parietal region since childhood. The lesion had increased in size gradually and had become $30 \mathrm{~mm}$ in diameter. The lesion was diagnosed as malignant melanoma clinically and also dermoscopically. Sentinel node navigation using radioisotope revealed the metastases. We performed wide local excision and radical neck dissection, and the definite diagnosis of malignant melanoma and regional nodal metastases was made. TNM staging was stage?b, that is, pT4b, N2c, M0. The patient also had nine small black freckles on the trunk and extremities. All of them were excised, and eight lesions of them were diagnosed as malignant melanoma, because proliferation of atypical melanocytes and formation of abundant abnormal nests in epidermis were observed histopathologically. Chemotherapy was performed, but metastases of skin and visceral region were found in February, 2004. These lesions had put up strong resistance to chemotherapy and the patient died on June, 6th, 2004. Autopsy revealed multiple metastases including heart, kidneys, liver and large intestine and so on. No other family members suffered from malignant melanoma. The origin of this patient's melanoma remained unclear. 
Abstracts

\section{CD44 AND HYALURONATE EXPRESSION IN FOLLICULAR MUCINOSIS}

Gürkan Kaya, Eric Augsburger, Pierre Chavaz and Jean-Hilaire Saurat

Department of Dermatology, University Hospital of Geneva, Geneva, Switzerland

CD44 is the cell surface receptor for hyaluronate (HA). We demonstrated that the lack of CD44 expression in murine keratinocytes leads to an abnormal HA accumulation in the dermis, indicating an important role of CD44 in local HA metabolism in mouse skin. We also observed a decrease in the expression of epidermal CD44 in patients of lichen sclerosus et atrophicus which is potentially responsable for the dermal deposition of HA. We also showed that HA accumulation was associated with decreased expression of CD44 in epithelial proliferations in perifollicular solitary cutaneous myxoma. In this study we examined the follicular expression of CD44 and HA in the skin biopsy specimens of seven patients with follicular mucinosis by using CD44-specific antibodies and biotinylated HA-binding protein (HABP), respectively. No difference of CD44 expression was observed in the follicular keratinocytes when compared with those of unaffected interfollicular epidermis. The follicular zones of mucin deposition were strongly positive for HA. A weak interkeratinocyte staining pattern for HA was also observed in the interfollicular epidermis. However, HABP staining revealed a stronger reactivity in the follicular keratinocytes surrounding the mucin-accumulated areas compared to the interfollicular keratinocytes, indicating an active secretion of HA by follicle cells in follicular mucinosis.

\section{EXPRESSION OF CD44 AND ITS ISOFORMS IN ORAL LICHEN PLANUS}

Gürkan Kaya ${ }^{1}$, Hakan Kaya ${ }^{2}$, Eric Augsburger ${ }^{1}$ and Jacky Samson ${ }^{3}$, Jean-Hilaire Saurat ${ }^{1}$ and Tommaso Lombardi ${ }^{3}$

${ }^{1}$ Dept of Dermatology, Geneva, Switzerland,

${ }^{2}$ Dept of Orthodontics, Univ of Istanbul, Turkey, and

${ }^{3}$ Div of Stomatology, Univ of Geneva, Switzerland

CD44 is a transmembrane glycoprotein which has multiple isoforms resulting from alternative splicing of ten variant exons (v1-v10). Standard CD44 (CD44s) is the cell-surface receptor for hyaluronate and is involved in lymphocyte homing and activation. The function of the variant CD44 isoforms (CD44v) is unknown. Recently we observed an altered expression of CD44s and CD44v in different inflammatory skin diseases. In cutaneous lichen planus (LP) CD44s and CD44v are expressed in the epidermis whereas dermal lymphocytes express strongly CD44s and weakly CD44v3 and are negative for CD44v. In this study we examined the expression of CD44s and CD44v (CD44v3, CD44v4, CD44v5, CD44v6, CD44v7, CD44v7v8 and CD44v10) in active oral LP lesions by immunohistochemistry. CD44s was highly expressed by keratinocytes and dermal lymphocytes. Epithelial expression of CD44v4, CD44v7, CD44v7v8 and CD44v10 was decreased or absent. Biopsies did not contain CD44v3, CD44v7, CD44v7v8 and CD44v10-positive lymphocytes. Lymphocyte expression of CD44v4, CD44v5 and CD44v6 was observed. Unlike CD44v4 and CD44v5, CD44v6 was expressed by large quantities of lymphocytes. Our results show that the expression of CD44v in oral LP is different from cutaneous LP, which suggests that differential expression of CD44v may play a role in the generation of LP in different locations.

\author{
CONGENTIAL NEVUS MIMICKING MELANOMA IN \\ INFANCY \\ P. Keehan, B. Rye and C. Cockerell \\ Department of Dermatology, University of Texas Southwestern \\ Medical Center, Dallas, TX, USA
}

A five-month old girl presented with a $5 \mathrm{~cm}$ brown patch on her right lower leg. The lesion had a $6 \mathrm{~mm}$ area of hyperpigmentation that was removed by shave biopsy for pathologic diagnosis. The clinical diagnosis at the time was that of congenital nevus but the multicolored appearance of the lesion raised the index of suspicion for melanoma. Histology revealed junctional activity with melanocytes distributed in the dermis including migration around appendages and among collagen bundles as seen in congenital nevi. However, there were some unusual features that warranted careful analysis. The lesion was asymmetric, with numerous single melanocytes in confluence at the dermo-epidermal interface. Single cells and nests were noted in the epidermis. Our case illustrates a congenital nevus with atypical patterns easily confused with those of melanoma. Our lesion exhibited pagetoid melanocytes, which is considered virtually diagnostic of melanoma. However, these melanocytes were monomorphous as opposed to the pleomorphic appearance found in melanoma. Additionally, there was evidence of cellular maturation in the dermis as well as a lack of variance in nest size, which favors the diagnosis of congenital nevusl.

\section{NODULAR GLEAR GELL HIDRADENOMA: A CASE REPORT WITH IMMUNOHISTOCHEMISTRY, GYTOGENETICS AND FLOW CYTOMETRY \\ Thaer Khoury, Paul Wallace, Sheila Sait, Richard and T. Cheney Department of Pathology, Roswell Park Cancer Institute.}

Background: Nodular Hidradenoma $(\mathrm{NH})$ is an uncommon neoplasm with controversial cell origin (myoepithelial, eccrine and more recently apocrine), and few cytogenetic and DNA flow Cytometry data. Material and Methods: We report a case of $\mathrm{NH}$ on the left ankle of a 77 year-old man. The tumor was located subcutaneously and measured $2.5 \times 2.0 \mathrm{~cm}$, with no throbbing, tenderness, edema, or groin lymphadenopathy. A wide local excision was performed. Immunohistochemistry using monoclonal antibodies to Smooth Muscle Actin (SMA), S-100 protein, p63 and Calponin were examined. A fresh central portion of the tumor was examined for karyotyping and DNA flow cytometry. Six months later the patient was healthy without local recurrence. Results: The tumor was positive for SMA, Calponin and p63 with focal staining for $\mathrm{S}-100$. The karyotype showed four aberrant, unrelated clones, $\mathrm{t}(1 ; 8)(\mathrm{p} 22 ; \mathrm{p} 23)$, del(1 1)(q14q23), t(1;10)(p13;q22) and del (6)(q23q26). DNA flow cytometry and cell cycle analysis showed that the tumor is $100 \%$ diploid with $6.55 \%$ S-phase. Conclusions: Expression of SMA, p63, Calponin, and S-100, which are myoepithelial markers, support a myoepithelial origin of this tumor. The multiclonal pattern observed in this neoplasm seems to reflect cytogenetic divergence during neoplastic progression. DNA diploidy with a low S-phase predicts a benign clinical course.

\section{AN UNUSUAL HEMATOLOGIC MALIGNANGY DERIVED FROM PLASMACYTOID DENDRITIC CELLS}

J.C. Kim, S.B. Peters, G. Frizzera and N.S. McNutt

Dermatopathology Division, Department of Pathology, Weill Cornell University Medical Center, New York, NY, USA

Recently reported cases of CD4+ CD56+ hematologic malignancies with a strong predilection for the skin correspond to the neoplastic 
counterpart of plasmacytoid dendritic cells. This rare, aggressive malignancy lacks pan-myeloid and pan-lymphoid markers and often presents with cutaneous lesions, splenomegaly, and involvement of lymph nodes and bone marrow. It has a poor prognosis, and many patients progress to acute myeloid leukemia. The proposed cellular origin is a CD56+ precursor cell related to plasmacytoid monocytes, which strongly expresses CD123 (IL-3Ra). We describe a 70 year-old man who presented with gray-brown truncal nodules and plaques, lymphadenopathy, and splenomegaly. His bone marrow demonstrated malignant CD4+ CD56+ mononuclear cells. Histologic sections of skin lesions showed an atypical infiltrate extending into the deep reticular dermis. Immunohistochemical staining of these cells for CD4 was diffusely positive. Moreover, the infiltrate strongly expressed CD56 and CD123 but showed only patchy or negative staining for other $\mathrm{T}$ and $\mathrm{B}$ cell markers. The combination of the patient's clinical presentation and biopsy results best fits the diagnosis of this newly characterized, distinct clinicopathologic entity described in recent literature as agranular CD4+ CD56+ hematodermic neoplasm, plasmacytoid dendritic cell acute leukemia, and tumor-forming accumulations of plasmacytoid monocytes associated with myeloid disorders.

\section{REGURRENT NEVUS PHENOMENON: A} CLINICOPATHOLOGICAL STUDY OF 328 PATIENTS

R King, D Ziegler, RN Page, MC Mihm Jr and PB Googe

Knoxville Dermatopathology Laboratory, University of Tennessee, Graduate School of Medicine, Knoxville, Vanderbilt Medical School, Nashville and Massachusetts General Hospital, Boston

Background: Recurrent nevi may histologically resemble malignant melanoma Design: Recurrent nevi with clinical history, original melanocytic neoplasm and recurrence were included. Proliferation marker Ki-67 was used when residual and recurrent nevus was present on the same slide. Results: There were 356 biopsies from 328 patients (ages 6 to 93 years $($ mean $=31$ ), female: male $=255: 101$, time to recurrence $1-63$ months $($ mean $=7.85)$ ). Sites: back $(170)$, abdomen (46), arm (46), leg (34), head and neck (26), chest (26), and buttock (8). Initial biopsies: 208 nevi, 142 dysplastic nevi, 1 blue, 1 pigmented spindle cell, 2 Spitz nevi, and 2 melanomas in situ. 3 patterns of recurrence were observed: 1) junctional melanocytic hyperplasia with epidermal effacement and scar, 2) compound melanocytic proliferation with epidermal effacement and scar, and 3) junctional melanocytic hyperplasia with retained retiform epidermis. Recurrent and residual nevi had similar proliferation rates. Conclusion: Recurrent nevi occurred more commonly in females and on the trunk. Cytologic atypia, asymmetry, and retention of retiform epidermis were worrisome histologic features. Clinical history, effacement of the epidermis and limitation of changes to the scar, distinguished recurrence from melanoma. Low proliferation rate may be a useful adjunct to distinguish recurrent nevi from melanoma.

\section{HIDRADENOGARCINOMA: CRITERIA FOR MALIGNANCY AND HYPOTHESIS OF AN APOEGCRINE ORIGIN}

C. Ko, F. Luo, A. Cochran and S. Binder

Department of Pathology, University of California, Los Angeles, Los Angeles, CA, USA

The immunohistochemical profile of hidradenocarcinoma, defined here as the malignant counterpart of hidradenoma, has not been well characterised. We evaluated the staining pattern of six cases of hidradenocarcinoma using antibodies to gross cystic disease fluid protein-15 (GCDFP-15), carcino-embryonic antigen (GEA), epithelial membrane antigen (EMA), S-100 protein, keratin AE1/3, cytokeratin 5/6, p53, bcl-1, bcl-2, and Ki67. All tumours were poorly circumscribed with clefting between tumour and stroma, evidence of poroid cells and cuticular cells, decapitation secretion, and increased mitoses with cords of tumour infiltrating through the adjacent desmoplastic stroma. The tumours stained with antibodies to GEA, S-100 protein, GCDFP-15, and EMA in no consistent pattern. All tumours studied stained positively for keratin AE1/3 and cytokeratin 5/6. Ki67 and p53 staining were strongly positive in 5 of 6 tumours. Bcl- 1 and bcl-2 staining were variable. Our study demonstrates that hidradenocarcinomas may have both apocrine and eccrine features within the same tumour and suggests that it may be most accurate to consider that these tumours originate from apoeccrine structures or stem cells with the capacity for pluripotential differentiation.

\section{COMPARISON OF SEBORRHEIC KERATOSES, INFLAMED SEBORRHEIC KERATOSES, AND INVERTED FOLLICULAR KERATOSES USING P53, BCL-1, AND BCL-2 \\ C. Ko and S. Binder \\ Department of Pathology, University of California, Los Angeles, Los Angeles, CA, USA}

While cell cycle markers have been used to differentiate benign versus malignant lesions and to classify malignant lesions, benign keratoses have not been well studied using such markers, and the relation of the cell cycle to inflammation or irritation of benign keratoses is unclear. We compared the immunohistochemical staining patterns of 10 seborrheic keratoses, 10 inflamed seborrheic keratoses, and 10 inverted follicular keratoses using antibodies to p53, bcl-1, and bcl-2. Staining with antibodies to p53 was increased in inverted follicular keratoses compared to inflamed or non-inflamed seborrheic keratoses. Bcl-1 staining was similar in all lesions. A population of bcl-2positive dendritic cells was seen within the epidermal portion of inverted follicular keratoses. Keratinocyte bcl-2 staining was higher in seborrheic keratoses compared to the other two keratoses. Bcl-2 may be increased in seborrheic keratoses as an anti-apoptotic mechanism while increased p53 may trigger apoptosis in inverted follicular keratoses.

\section{HISTOPATHOLOGIC DIFFERENTIAL DIAGNOSIS OF SUBEPIDERMAL CUTANEOUS BLISTERS AND EROSIONS AT AUTOPSY}

C. Kovarik, D. Stewart, C. Cockerell and J. Barnard

Departments of Dermatology and Pathology, University of Texas Southwestern Medical School, Dallas, TX, USA

The histopathologic differential diagnosis of subepidermal blisters is broad and most commonly includes primary dermatologic diseases (i.e. bullous pemphigoid), secondary blistering conditions (i.e. bullous lichen planus), and drug reactions (i.e. toxic epidermal necrolysis); however, when examining blister specimens taken during autopsy, several other entities need to be added to the differential diagnosis. For one year, we biopsied cutaneous blisters and erosions found during autopsies performed at the Dallas County Medical Examiner's Office and examined them histologically. The objective of this study was to determine the primary cause of blisters and erosions seen at autopsy, characterize them histologically in order to allow differentiation from other blistering conditions, and emphasize causes of subepidermal blisters that are often not considered in the differential diagnosis. We present eight representative cases in order to illustrate the following points. The majority of blisters and erosions examined 
were subepidermal and secondary to physical causes, such as burns, defibrillation, submersion, fetal maceration, and decomposition. Although primary skin conditions need to be considered when examining blisters and erosions at autopsy, the most common entities are secondary to physical causes, and these need to be included in the differential diagnosis in order to obtain an accurate diagnosis.

\section{ATYPICAL NEUROFIBROMA WITH BIZARRE GIANT CELLS}

Arni K Kristjansson, and Mary Stone

Department of Dermatology, University of Iowa, IA, USA. Zsolt Argenyi, Department of Dermatology, Univeristy of Washington, Seattle, WA, USA

A 27-year-old woman presented with a $5 \mathrm{~mm}$ papule on her cheek. She had no family history or stigmata of neurofibromatosis. Biopsy showed a diffuse dermal proliferation of spindle cells embedded in a slightly myxomatous stroma which entrapped adnexae. They displayed cytologic atypia with hyperchromatic nuclei and bizarre multinucleated giant cells. No mitosis were identified. Immunohistochemistry showed the spindle cells to be S-100 and collagen IV positive, but MART-1 negative. The differential diagnosis included desmoplastic melanoma, malignant peripheral nerve sheath tumor (MPNST) and atypical neurofibroma. Both atypical neurofibroma and desmoplastic melanoma are S-100 positive, show variable atypia and can have bizarre multinucleated giant cells. However, the lack of epidermal melanocytic hyperplasia, mitosis and the positive collagen type IV staining, favor nerve sheath differentiation. In neurofibromatosis, neurofibromas may undergo malignant degeneration resulting in MPNST. Whether atypical neurofibroma represents a transitional stage is unknown. Mitotic activity and necrosis strongly suggest MPNST. The biologic potential of cutaneous atypical neurofibroma has not been well studied, but they appear to behave in a benign fashion. This case demonstrates the diagnostic challenge of atypia in neurofibromas and highlights the need for further studies on the behavior of these rare variants.

\section{REGURRENT NEUTROPHILIC EGCRINE HIDRADENITIS}

M. Kucenic ${ }^{1}$, G. Fraga ${ }^{1}$, D. Belsito ${ }^{1}$, J. Patterson ${ }^{2}$ and J. Ashby ${ }^{1}$ Department of Dermatology, University of Kansas Medical Center, Kansas City, KS, USA ${ }^{1}$; Department of Dermatology and Pathology, University of Virginia, Charlottesville, VA, $\mathrm{USA}^{2}$

A 34-year-old female with acute myelogenous leukemia underwent induction chemotherapy with cytarabine (Ara-c) and idarubicin. Seven days later, while on broad spectrum antimicrobial therapy, she simultaneously developed fever coupled with painful, pruritic, and erythematous plaques on the face, neck, and chest along with marked edema and erythema of both palms and one sole. With negative blood cultures and a skin biopsy showing epithelial cell vacuolar degeneration and neutrophilic infiltrates surrounding eccrine gland coils, a diagnosis of neutrophilic eccrine hidradenitis (NEH) was rendered. Treatment with oral dapsone rapidly cleared the eruption. During her first consolidation with Ara-C and idarubicin four weeks later, a similar febrile cutaneous eruption occurred and again dapsone therapy led to resolution. For subsequent consolidation courses, premedication with dapsone was instituted and the patient remained eruption-free. NEH is a rare and often misdiagnosed disorder. Multiple chemotherapy medications have been implicated as etiologic agents including Ara-c, cyclophosphamide, topotecan, bleomycin, and mitoxantrone. Current oncologic induction-consolidation protocols carry the potential for recurrent NEH in predisposed patients. As this is only the second report of prophylactic dapsone therapy for $\mathrm{NEH}$, further investigation into this topic is warranted.

\section{HISTOLOGIC COMPARISON OF SCLEROMYXEDEMA AND NEPHROGENIC FIBROSING DERMOPATHY}

C. Kucher, X. Xu, T. Pasha and R. Elenitsas

Departments of Pathology and Dermatology, University of

Pennsylvania Medical Center, Philadelphia, PA, USA

Background: Nephrogenic fibrosing dermopathy (NFD), can be strikingly similar, if not indistinguishable, histologically from scleromyxedema. Both disorders consist of a dermal and subcutaneous proliferation of fibroblast-like cells with mucin production. NFD has been shown to contain an increased number of dual positive CD34 and procollagen cells, as well as an increased number of factor XIIIa positive cells. To our knowledge, there has been no direct comparison between the histologic and immunophenotypic properties of scleromyxedema and NFD. Design: Nine biopsies from eight cases of NFD and eight biopsies from eight cases of scleromyxedema were identified. Standard immunohistochemical techniques for antibodies toward CD34, procollagen I, CD31, SMA, and factor XIIIa were applied. A colloidal iron stain was used to evaluate for dermal mucin. Amount of antibody expression and mucin production were then evaluated using a grading scale of $0-3$. Results: Both NFD and scleromyxedema were found to have similar immunophenotypic expression. Both demonstrated increased numbers of CD34 and procollagen-positive fibroblast-like cells, as well as increased numbers of factor XIIIa positive cells. Increased vessels and stromal mucin were identified in both diseases without significant differences. Conclusions: Although scleromyxedema and NFD are clinically distinct diseases, theirÂ morphologic and immunophenotypic appearances are likely to be similar.

\section{RECALCITRANT ULCER CAUSED BY EMBEDDED PAPIER-MACHE}

J. Lee

Quest Diagnostics, Teterboro, NJ, USA

A 47-year-old sculptor presented with an ulcer on her neck. She is non-diabetic and had been on potassium iodide for hyperthyroidism. She developed a pruritic rash (?drug eruption), and her neck had been scratched repeatedly with papier-mache (PM)-covered fingers while she worked on PM sculptures for 3 consecutive months. Debrided material from neck ulcer revealed innumerable pieces of refractile $\mathrm{PM}$ in various shapes in the background of necrotic tissue and fibrinoid exudate, on polaroscopic examination of $\mathrm{H} \& \mathrm{E}$ sections. Stains for micro-organisms (for fungi, acid-fast bacilli, and actinomycosis) were negative. In an attempt to remove residual embedded PM, wide excision of the site and full-thickness skin graft was done. After initial successful take and healing, the area redeveloped an ulcer. Additional debridement and biopsy from the graft site showed PM clustered around the blood vessels in the reticular dermis. PM is a product of paper pulp commonly used in arts and crafts. Rare cases of lead exposure in PM workers have been reported in the literature, however, no cutaneous problems caused by PM have been reported to date.

\section{EPITHELIOID CELL HISTIOCYTOMA WITH GRANULAR GELLS \\ J. Lee \\ Quest Diagnostics, Teterboro, NJ}

Epithelioid cell histiocytoma (ECH) is regarded as a variant of fibrous histiocytoma. It is not readily recognized as fibrous histiocytoma 
because of its unconventional histologic features and its resemblance to Spitz nevus. The ECH reported herein was from a 50-year-old man who presented with an asymptomatic, $1.5 \mathrm{~cm}$, polypoid mass of unknown duration on his posterior shoulder. Histologic examination revealed epidermal collarette and dermal sheets of epithelioid cells with granular cytoplasm. Both fine and coarse granules were noted in the cytoplasm. They were PAS positive and diastase resistant. Many dilated vessels were also noted in the lesion. A panel of immunostains including S100, vimentin, CD68, and factor 13A was done. The lesional cells were reactive with vimentin, CD68, and factor 13A, and non-reactive with S100. These results favored exclusion of Spitz nevus and granular cell tumor, and supported the histologic impression of ECH. Both fine and coarse cytoplasmic granules were reactive with CD68. The latter finding supports that the cytoplasmic granules in this ECH represent lysosomes as in granular cell tumors.

\section{CHONDROMA OF THE TONGUE \\ J. Lee and B. Iskaros \\ Quest Diagnostics, Teterboro, NJ}

Soft tissue chondroma is a rare lesion that has been reported in various organs including brain, nasopharynx, larynx, lung, esophagus, liver, urinary bladder, fallopian tube, skin, and oral mucosa. We report a chondroma occurred on the tongue of a 38-year-old-woman. She presented with an asymptomatic, several-millimeter, firm papule on the lateral border of the tongue. She had been healthy otherwise, and the lesion had been present for about 9 to 10 months without change in size. Possibility of sialolith was considered clinically. Microscopic examination revealed a well-circumscribed, lobular, subepithelial lesion composed of mature hyaline cartilage. No cytologic atypia of chondrocytes was noted. The overlying squamous mucosa was unremarkable. Chondromas may occur in association with Maffucci's syndrome, Ollier's disease, CREST syndrome, and Carney's triad. However, in most cases, such as in this case, it is solitary and not associated with the preceding entities.

\section{PRIMARY CUTANEOUS NEUROENDOCRINE CELL TUMOR (MERKEL CELL CARCINOMA) WITH PROMINENT TUBULO-GLANDULAR FEATURES, MIMICKING ECGRINE CARCINOMA \\ Ning $\mathrm{Li}^{1} \mathrm{MD}$, Paula Zook ${ }^{2} \mathrm{MD}$, Zsolt Argenyi ${ }^{1,2} \mathrm{MD}$ \\ Departments of Pathology ${ }^{1}$ and Dermatology ${ }^{2}$, \\ University of Washington Medical Center, Seattle, WA, USA}

Although cutaneous Merkel cell carcinoma (MCG) may show divergent differentiation including tubular/glandular, squamous, eccrine, and rhabdomyoblastic differentiation, a diffuse glandular pattern is exceedingly rare. Herein, we present a case of MCG with prominent tubulo-glandular differentiation, which precluded a definitive diagnosis in the initial punch biopsy. The patient was a 43 year-old male with 5-6 weeks history of a rapidly growing plaque at his back. A punch biopsy revealed an infiltrating lesion consisting of tubulo-glandular structures lined by hyperchromatic basaloid cells, along with some angulated, basaloid tumor nests embedded in a desmoplastic stroma. Immunostains revealed that the tumor cells were strongly positive for chromogranin and BerEP4, variably positive for CK7 and CK5/6, but negative for CK20, synaptophysin, S-100, EMA, GCDFP-15, ER/PR, TTF1 and CEA. The reexcision specimen showed multifocal epidermotropism and typical finely stippled nuclear chromatin. Although the lack of CK20 staining is unusual, the histologic characteristics along with the remaining immunohistochemical studies favor the diagnosis of a primary cutaneous neuroendocrine cell carcinoma
(MCC) over the variants of microcystic adnexal carcinoma or basal cell carcinoma with neuroendocrine differentiation. Our case illustrates that prominent glandular differentiation may occur in MCG, and sometime create difficulty in definitive diagnosis in small biopsy specimen.

\section{KERATINOCYTE DYSPLASIA IN HEMATOPOIETIC STEM CELL TRANSPLANT RECIPIENTS IN THE DAY 28 TO 84 POST TRANSPLANT PERIOD. \\ Ning $\mathrm{Li}^{1} \mathrm{MD}$, Zsolt Argenyi ${ }^{1} \mathrm{MD}$, George Sale ${ }^{1,2} \mathrm{MD}$, David Myerson $^{1,2} \mathrm{MD}, \mathrm{PhD}$ \\ Departments of Pathology, University of Washington Medical Center, Seattle Cancer Care Alliance, Seattle, WA, USA}

Severe keratinocyte dysplasia (SKD) has been reported in the early post-transplant period of hematopoietic stem cell transplant (HCST) patients, with a frequency as high as $47.4 \%$. In the period less than 3 weeks post-transplant it was associated with cyclophosphamide conditioning. The purpose of our study was to determine the prevalence of SKD in a later post-transplant period, from 28 days to 84 days, and study the possible causes. The 2003 slide file (227 slides) of Seattle Cancer Care Alliance was examined for skin biopsies from patients who had undergone HCST. Twentytwo cases $(9.7 \%)$ showed SKD. A control group of 22 biopsies matched for days post-transplant and age were selected from the remaining 205 biopsies. SKD was associated with a busulfan conditioning regimen, $72.7 \%$ in the SKD group and $36.3 \%$ in the control group $(p=0.016)$. SKD was not associated with cyclophosphamide $(p=0.174)$, fludarabin $(p=0.263)$ or total body irradiation $(\mathrm{p}=0.50)$. Although active GVHD (grade 2 or 3 ) was more commonly seen in SKD group (45.5\%) than the control group $(22.2 \%)$, it did not show significant difference $(p=0.052)$. Our study showed that SKD developed in $9.7 \%$ of the skin biopsies from days 28 to 84 post-transplant, and was associated with busulfan conditioning regimen.

\section{CONGENITAL EROSIVE AND VESICULAR DERMATITIS WITH RETICULATED SCARRING \\ F. Lian, A. LI, S. Maberry and C. Cockerell \\ Dept of Dermatology, UT Southwestern Medical Center, Dallas, TX, USA}

Congenital erosive and vesicular dermatitis healing with reticulated scarring is a rare entity presenting in the early childhood. It is manifest as crusted erosions and vesicles that heal relatively rapidly forming unique reticulated scars. Cohen first reported this entity in 1985 and since then, only ten patients have been reported. We herein report an additional case of this extremely unusual disorder. A 23 month-old girl who was noted to have "rashes" and sores over her body at birth. Her twin sister was unaffected. She was born preterm at 35 weeks gestation but her developmental milestones have been within normal range. On exam she had depressed and somewhat macerated erosions and erythema primarily on her trunk, axillae, and ears. No vesicles or bullae were present. The skin biopsy showed neutrophilic and eosinophilic infiltrates with abundant crusting. Cultures were negative for infectious agents and direct immunofluorescent studies were also negative. Over the next two years she periodically developed erosions which healed quickly. However, the depressed reticulated scars have persisted and are more sensitive to sun exposure. This scarring disorder may be associated with mild ongoing blistering and must be distinguished from other congenital blistering disorders and immunobullous diseases. The pathophysiology remains unclear. 


\section{SPECTRUM OF HISTOLOGICAL FEATURES IN INFANTILE DIGITAL FIBROMATOSIS}

C. Liang, L. Capaldi, A. Ney, J. Gray, J. Katarincic, C. Lapidus and L. Robinson-Bostom

Department of Dermatology and Department of Orthopedics, Brown Medical School, Providence, RI, USA

Infantile digital fibromatosis (IDF), also called Reye tumor or inclusion body fibromatosis, is a benign proliferation of myofibroblasts, with characteristic perinuclear eosinophilic cytoplasmic inclusion bodies. The inclusions stain pink with hematoxylin-eosin, red with Masson's trichrome, and purple with phosphotungstic acid-hematoxylin. This intradermal neoplasm is formed by interlacing bundles of collagen and spindle cells. Positive immunohistochemical staining with vimentin, cytokeratin, desmin, and actin indicate myofibroblastic cell origin. Clinically, patients present at birth or infancy with asymptomatic smooth, flesh colored nodules, typically on lateral or dorsal digits. IDF is a benign neoplasm that may spontaneously regress and treatment is observation. Lesions recur in $60 \%$ of excisions; surgery is only recommended in cases of functional impairment or aggressive growth. We report three cases of IDF. The patients, ranging 8 months to 8 years old, presented with pink to flesh colored nodules on the feet and fingers. A spectrum of histological features was observed. All cases revealed dermal proliferation of spindle cells with blunt nuclei and eosinophilic intracytoplasmic inclusions. Pattern of spindle cells ranged from loose haphazard arrangement to well-organized interlacing bundles. The quantity of eosinophilic inclusions varied. For diagnostic and treatment purposes, all three patients underwent excision, with subsequent recurrence in two cases.

\section{CUTANEOUS ROSAI-DORFMAN DISEASE}

L. $\operatorname{Lin}^{1}$ and W. F. Bergfeld ${ }^{1,2}$

Departments of Anatomic Pathology ${ }^{1}$ and Dermatology ${ }^{2}$, Cleveland Clinic Foundation, Cleveland, Ohio

Rosai-Dorfman disease, or sinus histiocytosis with massive lymphadenopathy (SHML), is a rare benign disease of unknown etiology that involves the cervical lymph nodes. Histologic findings include emperiopolesis of lymphocytes and S-100 protein positivity. Extranodal involvement occurs in $30-40 \%$ of cases, often involving skin. However, cutaneous Rosai-Dorfman disease without lymph node involvement is uncommon. We describe a case of cutaneous Rosai-Dorfman disease and discuss its clinical and histopathologic findings. A 69-year-old Caucasian male presented with a two-year history of a slowly enlarging violaceous mass on the mid-upper abdomen. He had a history of renal cell carcinoma two-year ago and was asymptomatic without any systemic symptoms. Physical examination revealed a large, violaceous mass of mid-upper abdomen with several violaceous nodules surrounding the periphery. There was no palpable lymphadenopathy. Punch biopsy showed diffuse dermal infiltrates composed of central xanthomatous cells surrounded by lymphoplasmacytic cells. Focal germinal center was noted. Immunostaining revealed strong S-100 and CD68 reactivity of central xanthomatous cells and kappa and lambda polyclonal lymphoplasmacytic cells. Special stains for infectious disorder were negative. Cutaneous RosaiDorfman disease is a benign reactive process, often under-recognized due to lack of systemic symptoms and lymphadenopathy. A high index of diagnostic awareness is required for its recognition.

\section{CUTANEOUS LEISHMANIASIS IN AN AMERICAN SOL-} DIER WHO RETURNED FROM IRAQ - A CASE REPORT T.Y.D. Lin, C. Parrish, A. Bussian and E. Omura

Department of Dermatology, University of Alabama at Birmingham, Birmingham, AL, USA; P. Weina, Walter Reed Army Institute of Research, Silver Spring, MD, USA
A 27-year-old male soldier who was stationed in Iraq from 3/03 to 9/ 03 presented to a local dermatologist in 4/04 with several red slightly raised non-ulcerated nodules over the right wrist and ventral arm developed 2-3 months prior to the visit. The clinical impression was sporotrichosis. A punch biopsy was performed on a subcutaneous nodule and showed a granulomatous inflammatory process with scattered loosely organized clusters of epithelioid histiocytes palisading around dermal mucin, mainly involving the papillary and mid dermis. A diagnosis of granuloma annulare was suggested. Intralesional triamcinolone was used and the lesions initially improved but later recurred. However on deeper sections, there were scattered granulomas with a dominant necrotizing granuloma consisting of epithelioid histiocytes palisading around a central area of necrosis surrounded by lymphoplasmacytic and neutrophilic infiltrates. In 5/04, he presented to the Birmingham VA Medical Center with similar lesions. A repeat biopsy again showed a necrotizing granulomatous inflammation. In addition, numerous intracytoplasmic organisms with kinetoplasts were present within histiocytes. The findings were consistent with leishmaniasis due to L. major as confirmed by polymerase chain reaction at Walter Reed Hospital. As more US soldiers return from the Middle East, previously rare cutaneous leishmaniasis will be seen more commonly.

\section{CDIA RICH INFLAMMATORY STAGE OF LICHEN SCLEROSUS ET ATROPHICANS MIMICKING MYCOSIS FUNGOIDES AND EXTRAMAMMARY PAGET'S DISEASE \\ F. Luo, C. Ko and S. Binder \\ Department of Pathology and Lab Medicine, UCLA David Geffen School of Medicine, Los Angeles, CA, USA}

We report a case of inflammatory stage vulvar lichen sclerosus et atrophicans (LSA) with histopathologic features simulating both patch stage mycosis fungoides (MF) and extramammary Paget's disease. The patient is a 79-year-old woman with an erythematous plaque on her vulva. A punch biopsy was performed that revealed mild psoriasiform hyperplasia of the epidermis with spongiosis, marked interface vacuolar alteration, scattered individual necrotic keratinocytes and Civatte bodies. A band-like mixed inflammatory infiltrate was present in the papillary/upper dermis with prominent intraepidermal lymphocytes. Some intraepidermal lymphocytes showed atypical nuclear contours simulating the cells seen in MF. The presence of numerous intraepidermal epithelioid cells with a pagetoid distribution raised the possibility of extramammary Paget's disease. A panel of immunohistochemical (IHC) stains showed a reactive lichenoid mixed population of lymphocytes with numerous intraepidermal Langerhans cells. This confirmed the diagnosis of the inflammatory stage of lichen sclerosus. The presence of prominent epithelioid Langerhans cells with activated small lymphocytes represents an immune response in LSA and could cause diagnostic confusion with neoplastic conditions. IHC staining can confirm cell type and rule out MF and extramammary Paget's disease.

\section{FATAL SUBCUTANEOUS PANNICULITIS-LIKE T-CELL LYMPHOMA (SPTCL) WITH INTERFACE CHANGE AND DERMAL MUCIN, A DEAD-RINGER FOR LUPUS ERYTHEMATOSUS}

L. Ma, B. Bandarchi and E. J. Glusac

Department of Pathology and Dermatology, Yale New Haven Hospital, Yale University, School of Medicine, New Haven, CT, USA

We report a 48-year-old male who presented with ulcerated plaques and nodules of the lower extremities. Skin biopsies revealed a dense 
Abstracts

lymphocytic infiltrate involving the dermis and the subcutis in a lobular and septal pattern. No overt cytological atypia was present. Notably, several features resembling lupus erythematosus were present, including vacuolar interface change and abundant dermal mucin deposition. The patient developed pulmonary nodules, and a lung biopsy showed a perivascular and interstitial lymphoid infiltrate without overt atypia. The cutaneous and pulmonary lymphoid infiltrates showed similar immunohistochemical profiles: CD3+CD4 -CD8+/-CD56+. Monoclonal rearrangements of T-cell receptor gamma gene with similar migration patterns were identified from both locations. The patient developed fatal hemophagocytic syndrome, involving liver, spleen, lymph nodes, and bone marrow. This case is amongst rare reports of subcutaneous panniculitis-like T-cell Lymphoma (SPTCL) with systemic involvement.

\section{GUTANEOUS AND SYSTEMIC PLASMACYTOSIS}

L. Ma, A. Leonard, H. Kamino and S. Meehan

Department of Dermatology, New York University Medical Center, New York, NY, USA

A 67-year-old Chinese man presented with a longstanding history of asymptomatic, brown-red macules that diffusely involved his trunk and the extremities and was associated with generalized lymphadenopathy. His serum protein electrophoresis revealed a polyclonal hypergammaglobulinemia. No monoclonal Bence Jones protein was detected in the urine. Flow cytometry of his peripheral blood revealed a normal polyclonal population of $\mathrm{B}$ and $\mathrm{T}$ cells. Histopathologic examination demonstrated a dense nodular infiltrate of lymphocytes and numerous mature plasma cells in the reticular dermis. Immunoperoxidase studies for kappa and lambda light chains failed to demonstrate clonality. In addition, immunoglobulin gene rearrangement studies failed to reveal a monoclonal band. A bone marrow biopsy showed no abnormality. Cutaneous and systemic plasmacytosis is a rare lymphoproliferative disorder that is an important consideration in the differential diagnosis of cutaneous infiltrates in which plasma cells predominate.

\section{NODULAR HIDRADENOMA ARISING IN A NEVUS SEBACEUS OF JADASSOHN}

J. Michael Magill, Jr., Sima M. Jain, Amy Devore, Gloria Chin and Ashraf M. Hassanein

Department of Pathology, University of Florida College of Medicine, Gainesville, FL, USA

Nevus sebaceous of Jadassohn, a cutaneous hamartoma, results from disordered development of epidermal, follicular, sebaceous and apocrine differentiation that typically appears as a yellow verrucous plaque on the scalp or face. We present a 36-year-old white male with a congenital, nodular lesion along the hairline of his forehead, which had been traumatized repeatedly during hair grooming. Clinically, it was a well-demarcated, curvilinear, erythematous, non-tender plaque with no umbilication, ulceration or discharge. The periphery had a circumferential tan-yellow, raised, waxy serpiginous border. Microscopically, the specimen had epidermal papillomatous hyperplasia with dilated infundibula of irregular mature sebaceous lobules, and mid-dermal apocrine gland lobules. Within the hamartoma, was a well-circumscribed, multi-lobulated dermal tumor with epidermal and follicular attachments, focal ductal differentiation and cystic changes. Two distinct cell types were present; in the center were large polyhedral cells with focal clear cytoplasm, and at the periphery were spindle-shaped cells with basophilic cytoplasm. These findings were typical of nodular hidradenoma arising within a nevus sebaceous of Jadassohn, a rare event with only seven cases previously documented in the literature.
PHOTOSENSITIVITY IN AN HIV-INFECTED MAN

D. Manion, P. Tamjidi, B. Santmyire-Rosenberger and E. Dugan Departments of Dermatology and Pathology, Washington Hospital Center, Washington, DC, USA

Since the emergence of the AIDS epidemic, various associated skin lesions have been recognized. One interesting phenomenon is chronic photosensitivity dermatitis. We report the case of a 44 -year old homeless male with a 14-year history of HIV and a CD4 count of 350. He presented to us with a 5-month history of a pruritic photodistributed eruption. Physical examination revealed that he had erythematous, scaly, lichenified, and crusted plaques involving the face, neck, upper chest, and extensor extremities. Sparing of the areas under his watch and bracelet was found. A skin biopsy specimen showed psoriasiform dermatitis with an overlying thick, impetiginized, and hemorrhagic crust. There was prominent single cell necrosis throughout all epidermal layers. A lichenoid dermal infiltrate consisted mainly of lymphocytes and eosinophils. The patient's presentation was consistent with a photosensitive psoriasiform interface dermatitis. The pathogenesis of this photodermatitis is unclear. Proposed mechanisms include the formation of a neoantigen due to UV exposure, depressed levels of endogenous scavengers leading to increased oxidative stress, and the onset of a pellagra-like dermatosis due to niacin-tryptophan deficiency. It is likely that several of these processes are acting in concert to cause this photoeruption.

\section{GUTANEOUS T-GELL LYMPHOMA OF 12 YEARS DURATION INITIALLY PRESENTING IN A TWO YEAR OLD}

L. Mark and S.D. Billings

Departments of Dermatology and Pathology, Indiana University School of Medicine, Indianapolis, IN, USA

A 14-year-old Caucasian girl presented with an eczematous patch on the left proximal inner arm of 12 years duration. She had previously been treated with emollients and topical steroids without complete resolution. The patient denied constitutional symptoms but did note eczematous patches on her buttocks of one-year duration and new eczematous patches on her inner left thigh and right inner upper arm. Upon exam, all lesions were slightly scaly without induration. The primary lesion was a poikilodermatous $4 \times 10 \mathrm{~cm}$ rough plaque. No lymphadenopathy was noted. A total of five lesional biopsies were obtained. All of the biopsies demonstrated a superficial infiltrate of atypical lymphocytes with cerebriform nuclei and prominent epidermotropism including the formation of Pautrier's microabcesses. The diagnosis of mycosis fungoides was rendered. This case is significant for its onset at two years of age, the benignity of its course over a 12 year period and the long duration before definitive diagnosis was obtained. Mycosis fungoides needs to be considered in the differential diagnosis of recalcitrant eczematous disease even in young children.

\section{HISTOLOGIC FEATURES OF MELANOCYTIC NEVI IN OLDER PATIENTS}

Martinka M

Department of Pathology and Laboratory Medicine, University of British Columbia; Bruecks AK, Trotter MJ, Department of Pathology and Laboratory Medicine, University of Calgary and Calgary

Laboratory Services.

The histology of melanocytic nevi in elderly patients often differs from nevi in younger adults. Our hypothesis was that nevi in patients ${ }^{3}$ 60 yrs would demonstrate histologic patterns different from nevi in younger adults. Biopsies of nevi $(\mathrm{n}=214)$ from 172 patients $^{3} 60 \mathrm{yrs}$ 
(mean age $69 \pm 7 \mathrm{yrs}$ ) were examined by three dermatopathologists and a consensus diagnosis was rendered. Control specimens $(n=82)$ from 58 patients $20-40 \mathrm{yrs}$ (mean age $31 \pm 5 \mathrm{yrs}$ ) were evaluated. Compound melanocytic nevi were commoner in younger patients $(37 \%)$ versus older patients $(12 \%)(\mathrm{p}<.0001)$. Junctional melanocytic nevi were more frequently diagnosed in older patients $(10 \%$ versus $3 \% ; \mathrm{p}=.027)$ and a lentiginous, often heavily pigmented, growth pattern was common (12\% of nevi) vs control group $(3 \% ; \mathrm{p}=.028)$. Atypical ("dysplastic") nevi had a similar frequency at all ages, however $12 / 214$ nevi in elderly patients (6\%) exhibited marked atypia with features suggesting melanoma in-situ. We conclude that benign junctional nevi are relatively common in elderly patients and that a lentiginous, heavily pigmented growth pattern, typically associated with younger patients, is often seen in both junctional and compound nevi in this older age group. This pattern must be differentiated from dysplastic nevus and melanoma in-situ, which they may clinically resemble.

\section{EXTRANODAL ANAPLASTIC LARGE CELL LYMPHOMA OF THE EYELID}

V. Mathur, H.K. Park and J. Finley

Department of Pathology, East Carolina University School of

Medicine and R. Lewis, Eastern Dermatology and Pathology

A sixty-nine-year-old male patient, without a significant prior medical history, presented with a rapidly enlarging, $2 \mathrm{~cm}$ non-tender nodule on the right lower eyelid. The lesion persisted in spite of an incision and drainage. Following an excision, the histology revealed a diffuse infiltrate of atypical mononuclear cells within the dermis. The cells were large, monomorphic, with irregular to convoluted nuclei, prominent nucleoli, amphophilic cytoplasm. They were very mitotically active. Immunohistochemical stains for CD45, CD3, and CD30 were strongly positive. Pancytokeratin, CD20, and ALK (Anaplastic Lymphoma Kinase fusion protein) were negative. The histopathologic diagnosis was CD30 (Ki-1) positive anaplastic large cell lymphoma. The margin was positive for involvement by lymphoma. The lesion was re-excised with no evidence of residual involvement by lymphoma. A primary cutaneous anaplastic large cell lymphoma was favored over systemic involvement based on the clinical presentation of a single eyelid nodule and ALK negativity. He was referred to a hematology-oncologist for further management. Anaplastic large cell lymphoma is a distinctive type of malignant lymphoma with a relatively favorable prognosis. It frequently involves the skin, however, to the best of our knowledge, presentation of this lymphoma type as a single lesion on the eyelid is extremely rare.

\section{NEPHROGENIC FIBROSING DERMOPATHY WITH OSTEOID METAPLASIA \\ V. Mathur, H.K. Park and J. Finley \\ Department of Pathology, East Carolina University and C.L. Smith, Eastern Dermatology and Pathology}

Nephrogenic fibrosing dermopathy is a rare and recently described disease which occurs in patients with a history of renal disease and is often associated with hemodialysis or renal transplants. The usual presentation is multiple indurated plaque lesions resembling scleromyxedema on the extremities and trunk. Nephrogenic fibrosing dermopathy has been described in association with calciphylaxis and metastatic calcification, however, to our knowledge, the occurrence of osteoid metaplasia has not been previously reported. We report a case of nephrogenic fibrosing dermopathy with osteoid metaplasia in a 78year-old female patient with chronic renal failure on dialysis. The patient had a relatively acute onset (a few months) of lesions with irritation and pruritis of the lower extremities. Biopsies showed fibrous thickening of the dermis with islands of osteoid tissue with osteoblastic rimming. Calcification and lamellar bone formation were not seen. An Alcian blue stain demonstrated increased dermal mucin. Elastic stains showed prominent elongated elastic fibers between collagen fibers and within osteoid areas. The differential diagnosis included scleromyxedema and other fibrotic disorders. The pathogenesis of this disorder is unknown. Although rare patients improve with correction of renal abnormalities, currently no effective treatment is available.

\section{GUTANEOUS SPARGANOSIS: A CASE REPORT AND LITERATURE REVIEW}

M. Matthews, B. Murphy, J. Wharton, F. Lian, R. Oracion, O. Sunga and C. Cockerell

Department of Dermatology, University of Texas Southwestern Medical Center at Dallas, Dallas, Texas USA

Sparganosis is a helminthic infection caused by the migrating larvae of the cestode genus Spirometra. Human infection is most commonly encountered in regions of the Far East where it has been associated with dietary consumption and medicinal usage of raw snake and frog tissue preparations or the drinking of contaminated water. Approximately 63 cases of sparganosis have been reported in the United States and reports of cutaneous sparganosis are very rare within the world literature. A case of cutaneous sparganosis involving the lateral abdominal region of a 54-year-old Filipino American women is discussed. Clinically the lesion presented as a cutaneous nodule. Skin punch biopsy revealed a sparganum possessing an undulating body wall with outermost microvillus layer overlying a thick tegument. Two thin smooth muscle layers and deeper subtegumental cells resided subjacent to the tegument. Calcareous corpuscles and intermixed mesenchymal tissues occupied the central regions of the larval worm. The inflammation consisted of a dense perivascular lymphohistiocytic infiltrate with scattered eosinophils and surrounding dermal fibrosis. Cutaneous sparganosis is rarely encountered in dermatology and dermatopathology practice, hence recognition of the clinical, histomorphological and patient history characteristics associated with this helminthic infection will facilitate in diagnosing this entity.

\section{CUTANEOUS GONGENITAL PLEXIFORM CELLULAR SCHWANNOMA: A SIMULANT OF MALIGNANT PERIPHERAL NERVE SHEATH TUMOR OF CHILDHOOD - A CASE REPORT AND LITERATURE REVIEW \\ M. Matthews, J. Raj, C. Charissi and C. Cockerell Department of Dermatology, University of Texas Southwestern Medical Center, Dallas, TX USA}

Cutaneous plexiform schwannoma is a rare multilobulated variant of benign schwannoma. When presenting as the cellular subtype with significantly increased proliferative rate, diagnostic consideration of a malignant peripheral nerve sheath tumor is prompted. However, follow up demonstrates a benign natural history with propensity for local recurrence and typical lack of association with neurocutaneous syndromes. These lesions most commonly occur as cutaneous and subcutaneous masses of the extremities in the first four decades. Only 9 cases of congenital tumors are reported. A case of congenital plexiform cellular schwannoma, presenting as a cutaneous mass measuring $3.5 \times 2.5 \times 2.0 \mathrm{~cm}$, excised from the left upper arm of a 16 month old female is discussed. Regions of increased cellularity and proliferation co-localized and were unassociated with nuclear anaplasia. Mitotic 
figures numbered 7 per $10400 x$ fields. The tumor cells were strongly immunoreactive for S-100 protein. The MIB-1index was multifocally $25 \%$ and p53 protein over expression was present in $50 \%$ of nuclei. Following excision with free margins the tumor locally recurred after 16 months. The recurrence was morphologically identical to the original lesion. Recognition of the clinical and morphological characteristics of this rarely encountered benign neoplasm will facilitate in diagnosing this entity.

\section{ATYPICAL COMPOUND NEVUS ARISING IN MATURE CYSTIC OVARIAN TERATOMA (DERMOID CYST) \\ J. McHugh and D. Fullen \\ Department of Pathology, University of Michigan Medical Center, Ann Arbor, MI, USA}

Mature cystic ovarian teratomas (MCOT) are the most common ovarian neoplasm and contain components of one or more embryonic germ cell layers. Ectodermal components are most common. Rarely (1-3\%), malignant transformation can occur in MCOT and are usually epidermoid, although multiple case reports of melanomas have been described. Four compound nevi and three blue nevi have also been reported; however, atypical (dysplastic) nevi have not been reported to our knowledge. A 28-year-old woman presented with a right ovarian mass. A $5 \times 4 \times 2.5 \mathrm{~cm}$ cystic ovarian mass was filled with hair and grumous keratinaceous debris. The lining was smooth with a $1 \mathrm{~cm}$ dark macule. Histologically, the mass was predominantly lined by epidermis and appendages. The pigmented lesion consisted predominantly of melanocytic nests irregularly disposed on sides and tips of distorted rete ridges, some with bridging between adjacent rete ridges, and junctional extension lateral to the small dermal component of nevus. Slight fibrosis invested some rete ridges. Moderate atypia was present. Although melanomas and melanocytic nevi have rarely been described previously in MCOT, we present the first atypical (dysplastic) compound nevus, to our knowledge. This case expands the spectrum of melanocytic lesions arising in MCOT.

\section{SPECTRAL IMAGING MICROSCOPY CAN DEFINE HYPERCHROMASIA IN SMALL LYMPHOID GELLS N.S. McNutt, R.M. Levenson* and S.B. Peters Department of Pathology, Weill Medical College of Cornell University, New York, NY and *CRI, Inc., Woburn, MA, USA}

Spectral Imaging Microscopy (SIM) is a technique for performing image-enabled spectroscopic analysis using a standard light microscope. We employed a prototype to a current, commercially available, spectral imaging system (NuanceTM, CRI, Inc.) that electrooptically selects narrow bands of light to be collected by a cooled, monochrome CCD camera. For each sample, images were taken every $10 \mathrm{~nm}$ from $420 \mathrm{~nm}$ to $720 \mathrm{~nm}$ to create a spectral image "stack." Analysis of the images with a prototype to the Nuance TM Analyze software (CRI, Inc.) gave both standard color (RGB) and spectral information at each pixel of the images. A representative 40x field was spectrally imaged from H\&E slides of 104 biopsy specimens of mycosis fungoides (MF). Optical densities and spectral properties of smalllymphocyteswere determined. Hyperchromatic cellswerepseudocolored to show their distribution. In addition to the important architectural features of MF, SIM can define whether hyperchromatic or normochromatic small lymphocytes are epidermotropic in the lesion by setting appropriate threshold values. Hyperchromasia can be attributed to increased nuclear eosinophilia or basophilia. These differences are difficult to estimate by eye. Since lymphoblasts have enlarged nuclei with dispersed chromatin, a combination of spectral and spatial data is required for their analysis.
DIRECT IMMUNOFLUORESCENGE RESULTS OF A CASE OF RAILWAY TRACK DERMATITIS

P. Mecca MD and J. A. Carlson MD

Department of Pathology, Albany Medical Center; J. Schwartz MD, Troy, NY

A 40 year-old female with sero-negative arthritis presented with annular and cord-like configurations (rope sign) of asymptomatic papules and plaques along her flanks, suspected to be granuloma annulare. Medications at the time of presentation included methotrexate, prednisone, Vioxx (rofecoxib), Arava (leflunomide) and Remicade (infliximab). High-potency topical corticosteroids did not alleviate the eruption. Biopsy was performed demonstrating a deep dermal, interstitial and focally palisading granulomatous infiltrate with eosinophils. Dermal lymphangiectases were present, and the epidermis was unaffected. Direct immunofluorescence (DIF) examination showed deep dermal, interstitial IgG, C3, and IgM deposits coating collagen bundles centered in the granulomatous infliltrate and IgG antinuclear keratinocyte reactivity. Serologic tested also revealed anti-nuclear antibodies. No other auto-antibodies were detected. Based on these findings, this patient was diagnosed with "interstitial granulomatous dermatitis (IGD) with cords and arthritis". Thirteen cases of IGD with cords were identified in a literature review of which $7 / 12$ (58\%) were sero-(rheumatoid factor)-negative, $3 / 5(60 \%)$ were anti-nuclear antibody positive, and 12/13 (92\%) had arthritis. One theory of pathogenesis is that immune-complex deposition in the dermis initiates a granulomatous reaction; DIF findings in this case support this proposed mechanism. IGD with cords is a singular clinicopathologic entity associated with sero-negative, non-erosive arthritis and antinuclear antibodies.

\section{ONYCHOBLASTOMA - HAMARTOMA OF THE NAIL} UNIT: A NEW ENTITY?

C. Misciali, P.A. Fanti, M. Iorizzo, BM Piraccini and A. Tosti

Department of Dermatology, University of Bologna, Italy

A 50-year-old Peruvian man presented with a $4-5$ years history of a $1,2 \times 0,7 \mathrm{~cm}$ subungual verrucous mass that extended from the proximal nail fold to the hyponychium of the 2nd left toe. Histology of the nail bed showed a nodular tumour with multiple structures, similar to the infundibulum of the follicles, and germ like aggregations often joined to a follicular papilla. Few scattered neoplastic cells were necrotic. The stroma surrounding the nodular aggregations was made up of delicate fibrillary bundles of collagen associated with numerous oval fibrocytes. The histological findings remind us a neoplasm with follicular differentiation: trichoblastoma. A tumor with these histological aspects on the nail unit has never been reported. We name this benign neoplasm, which probably represents an hamartoma, onychoblastoma. Differential diagnosis of this case includes basal cell carcinoma (BCC) of the nail unit which is rare and mostly localised in the nail folds. The main histopathologic findings of nodular BCC are asymmetry, ulceration and its poorly circumscription. The hair follicle and the nail share similar embryological and anatomical features and the occurrence of a tumor with follicular differentiation in the nail further demonstrates the common origin of these adnexal structures.

\section{LYMPHOEPITHELIOMA-LIKE CARCINOMA OF THE} SKIN: A CASE REPORT

T. Mitsuhashi, T. Tsuchida, E. Arai, S. Ban, T. Shimada, A. Kuramochi, T. Hirose and M. Shimizu Departments of Pathology and Dermatology, Saitama Medical School, Saitama, Japan 
Lymphoepithelioma-like carcinoma of the skin is a rare tumor with a microscopic resemblance to lymphoepitheliomatous carcinoma of the nasopharynx. We present a 93-year-old man with papules on the left auricle to the cheek that were gradually enlarged. By the time of a biopsy, it grew to a $5.0 \times 3.0 \times 2.8 \mathrm{~cm}$ dark red mass, and necrotic debris was attached to the surface. Histologically, a relatively welldemarcated, dermal-hypodermic multiple lobules were composed of irregular islands of atypical epithelial cells. The uniform tumor cells had moderate amounts of lightly eosinophilic cytoplasm and vesicular nuclei with one or two prominent nucleoli. A dense lymphocytic infiltrate was present within the neoplastic islands, obscuring the epithelial component. The neoplastic cells were unconnected to the overlying epidermis. Neither squamous nor glandular differentiation was present. Immunohistochemically, the neoplastic cells were positive for epithelial membrane antigen and cytokeratin, and negative for latent membrane protein 1. No Epstein-Barr virus-encoded RNA (EBER) was detected by in situ hybridization. The negativity for Epstein-Barr virus may be a help in the differential diagnosis from metastatic undifferentiated nasopharyngeal carcinoma, which is uniformly positive for EBER.

\section{THE HAIR EREGTOR MUSCLE: ORDER AND DISORDER OF THIS OFTEN FORGOTTEN STRUCTURE \\ Liliana Muñoz-Garcia and Aldo González-Serva \\ Ameripath New England and Department of Pathology, Boston \\ University, Boston, MA, USA}

Hair erector muscle (HEM) is everywhere in dermatopathology but has been less studied than other smooth muscle systems in the skin. HEM disorders are passive and active, the former related to the involvement, as a bystander fascicle, by nearby conditions. The muscle can be permeated by nevus cells, infiltrated or destroyed by keratoacanthoma and other malignancies, encroached by hemangiomas, suffused by erythrocytes in purpura and pyocytes in an abscess, incorporated into scars and entrapped and deformed by a dermatofibroma. Less passively, HEM can participate in follicular processes, insect bites, collagen-vascular diseases, syphilis, solar elastosis and amyloid. More proper to the muscle yet rarely encountered are myositis (dermatomyositis), poorly understood myopathies (atrophy, vacuolar changes, eosinophilic globules), deposit diseases (glycogenosis) and, even, rare dystrophies (Duchenne/Becker). Finally, other than HEM tumors (leiomyoma, leiomyosarcoma), hamartomatous conditions include disorganized/hypertrophic muscle in blue nevi, hyperplasia in Becker's nevus, and more substantial muscle mass increase as in smooth muscle hamartoma (including similar changes in mature port-wine stain) and in the rare congenital midline hamartoma. This presentation outlines orderly features and, mainly, compiles disorders of HEM, few of them a disease on its own but still a supplement to a gamut of many more noticeable entities.

\section{PALE CELL SQUAMOUS CELL CARCINOMA IN ASSOCIATION WITH PALE (CLEAR) CELL ACANTHOMA}

Liliana Muñoz-Garcia and Aldo González-Serva

Ameripath New England and Department of Pathology, Boston University, Boston, MA, USA

An 85-year-old woman developed a tumor on her left thigh, thought to be a squamous cell carcinoma (SCG). Microscopy revealed irregularly shaped, bulbous and deep aggregates of pale/clear cells emanating from the surface and reaching the lowermost dermis. This asymmetric mass of keratinocytes showed focal necrosis and dyskeratosis but no frank anaplasia, mitoses or infiltrative pattern. The interface with the dermis was blunt but irregularly undersurfaced. A more characteristic pale cell acanthoma (PCA) was colliding with it and consisted of a superficial, more regularly psoriasiform proliferation with thin suprapapillary plates and erosion but no necrosis. While it has been known that PCA can be unusual polypoid, large or atypical no author has proposed that some of the latter variants could represent a malignancy, more akin to verrucous carcinoma than to conventional SCC, due to the paucity of overt anaplastic features and the underestimation of pushing invasion and low cytologic atypia as defining traits of malignancy. One case of SCC in situ with PCA has been recently acknowledged but this is not what we are reporting: We propose that voluminous, misshapen, asymmetric or deep "PCAs" may be lowgrade carcinomas with pale cell differentiation and likely origin in a pre-existing PCA.

\section{METASTATIC ESOPHAGEAL CARCINOMA MASQUERADING AS INFLAMMATORY BREAST CARCINOMA}

C. L. Nebesio, M. Yuraitis, P.R. Helft, R.J. Goulet and S.D. Billings Departments of Dermatology, Pathology, Surgery, and Hematology and Oncology, Indiana University School of Medicine

Metastasis to the breast from extramammary neoplasms is rare. Such metastatic neoplasms can mimic inflammatory breast carcinoma clinically and histologically, presenting a diagnostic challenge. A 50year-old woman with a history of esophageal adenocarcinoma in clinical remission presented with rapid onset unilateral breast swelling with warmth and erythema. Based upon the clinical presentation, an underlying breast carcinoma was suspected. A punch biopsy revealed a poorly differentiated adenocarcinoma with extensive involvement of the dermal lymphatics. The clinical and histologic differential diagnosis included inflammatory carcinoma secondary to a primary breast carcinoma vs. metastatic esophageal adenocarcinoma to the skin of the breast. To resolve this question, immunohistochemical stains for estrogen and progesterone receptors and CDX-2 were performed. CDX-2 is an intestinal homeobox gene that is expressed in intestinal epithelium and tumors with intestinal differentiation including esophageal adenocarcinoma. CDX-2 expression has not been reported in breast carcinoma. The tumor cells were positive for CDX-2 but negative for both steroid receptors. The diagnosis of metastatic esophageal adenocarcinoma to the skin of the breast was confirmed. This case emphasizes the importance of confirming the tumor lineage in inflammatory carcinoma of the breast. CDX-2 can be a useful tool in establishing a gastrointestinal origin of cutaneous metastases.

\section{PRIMARY GUTANEOUS NATURAL KILLER GELL BLASTIC LYMPHOMA \\ A. Ney, J Carlsten, D Pomerantz, L Glasser, LJ Wang, P Rintels and L Robinson-Bostom \\ Departments of Dermatology, Pathology and Oncology, Brown Medical School, Providence, RI, USA}

An 81 yo male presented with several asymptomatic firm $1-5 \mathrm{~cm}$ red purple plaques on the trunk and lower extremities associated with a mild pancytopenia. Histological examination revealed a diffuse, monotonous dermal infiltrate of atypical medium sized cells with fine chromatin and scanty cytoplasm. Immunoperoxidase staining demonstrated positivity for CD 45, CD43, CD4, Bcl-2, and TdT; but was negative for cytokeratin, melan-A, CD30 and hematopoietic lineage specific markers. A subsequent bone marrow aspirate 
demonstrated a dense population of cells that were morphologically consistent with blasts. Immunophenotyping by flow cytometry revealed lesional cells that expressed CD56, GD4, CD7, CD5, HLA-DR and TdT. However, lineage specific markers for B-cells (CD19, CD20, cCD79a, and CD10), T-cells (sCD3 and cCD3), and myeloid cells (CD13, CD33, GD117, cGD13, CD14, GD41, CD61, myeloperoxidase, and alpha napthyl butyrate esterase) were not expressed. Molecular studies by PCR exhibited no evidence of T-cell receptor or heavy chain gene rearrangements. Collectively, these findings are consistent with a primary cutaneous blastic natural killer cell lymphoma. Blastic natural killer cell lymphomas are characterized by a high incidence of cutaneous involvement and an aggressive clinical course. Our patient responded dramatically to one cycle of CHOP chemotherapy with resolution of his cutaneous tumors.

\section{VESICULO-BULLOUS DERMATOMYOSITIS ASSOCIATED WITH MALIGNANT LYMPHOMA}

F. Ogawa, T. Tsuchida, E. Arai, A. Kuramochi, T. Mitsuhashi, S. Ban, Y. Shimizu, T. Hirose and M. Shimizu Departments of Pathology and Dermatology, Saitama Medical School, Saitama, Japan

Dermatomyositis is an inflammatory condition of the skin and muscles, and an underlying malignancy is noted in $10 \%$ or more of cases. Clinical features of dermatomyositis include increasing general fatigue and proximal (thighs and shoulders) muscle weakness accompanied by erythematous lesions of the skin. There have been several distinct types of dermatomyositis described. Here we describe a case of vesiculo-bullous dermatomyositis, which is a rare variant of dermatomyositis. A 49-year-old woman was admitted to our hospital with a painful erythematous vesicular eruption of the face, trunk and extremities. In addition, edema of the face and fever were observed. Clinically, dermatomyositis was considered because of typical skin rashes (Gottron's papules, periorbital heliotrope rash and poikiloderma) and serum creatine phosphokinase level of 1,031 IU/L. A skin biopsy was performed. Microscopically, subepidermal vesiculation with marked edema was present. Lymphoplasmacytic infiltration was also observed in the upper dermis. So far only a few case reports of vesiculo-bullous dermatomyositis have been reported in the literature. It should be kept in mind that dermatomyositis may present subepidermal vesiculation in order to avoid a misdiagnosis and unnecessary delayed treatment. Furthermore, an internal malignancy should be considered in such a variant of dermatomyositis.

\section{CLINICOPATHOLOGICAL AND} IMMUNOHISTOCHEMICAL STUDY OF ECGRINE POROCARGINOMA (MALIGNANT ECGRINE POROMA) F. Ogawa, E. Arai, T. Tsuchida, A. Kuramochi, T. Mitsuhashi, S. Ban, Y. Shimizu, T. Hirose and M. Shimizu Departments of Pathology and Dermatology, Saitama Medical School, Saitama, Japan

Porocarcinomas are thought to be differentiated into the acrosyringeal portion of the sweat duct, and they are relatively rare. It may develop from a long standing eccrine poroma undergoing malignant transformation or may arise de novo. We evaluated pathological and immunohistocheimical findings of six cases of eccrine porocarcinoma. Special stains (PAS and PAS with diastase) and immunohistochemical stainings (CEA, CAM5.2, CK7, bcl-2, p53 protein, and MIB-1) were performed. We observed asymmetrical architecture, cytological pleomorphism, prominent nucleoli, ductal differentiation, intracytoplas- mic lumina, mitotic activity, and melanin colonization. Three patients were male and four patients were female. The average age was 76 years. The time interval from tumor onset to treatment ranged from 6 months to 10 years. The mean size was $3.2 \mathrm{~cm}$, and the mean depth of invasion was $1.0 \mathrm{~cm}$. Four tumors were found on the head, one on the hip, and one on the foot. Regional lymph node metastases were noted in one case. Microscopically, luminal spaces and intracytoplasmic lumina were noted in all cases. Immunohistochemically, the mean MIB-1 index was 41\%. P53 immunoreactivity was detected in five cases. Interestingly, a case of porocarcinoma with lymph node metastases showed high mitotic figures as well as less lymphocytic infiltration.

\section{ATYPICAL LYMPHOCYTIC LOBULAR PANNICULITIS IN KOREA: FREQUENT EXPRESSION OF EPSTEIN - BARR VIRUS \\ CW Oh \\ Dept. of Dermatology, College of Medicine, GyeongSang National University, Chinju, S. Korea}

Although subcutaneous T-cell lymphoma is considered an aggressive form of lymphoma, some patients manifest a long waxing and waning phase unaccompanied by constitutional symptoms. Magro etal (2004) applied the term "atypical lymphocytic lobular panniculitis (ALLP)" to this distinctive form of lymphocytic panniculitis. Eight ALLP patients (five females and three males, mean age: $34.0 \pm 12.5$ ) were encountered from Jun 1999 to Nov 2003 at GyeongSang National University Hospital in Korea. Five patients (62.5\%) had skin lesions only and oral prednisone therapies were done. Two of five had no relapse but three showed a waxing and waning course till now. In three patients $(37.5 \%)$ presenting cytopenias and fevers, chemotherapies were done with prednisone, cyclophosphamide, vincristine, doxorubicine, bleomycin and/or adriamycin. Two of three led to complete resolution till now and included only one patient with clonal T-cell receptor-gamma rearrangements. In seven patients, serum EBV antibody studies were performed. Six of them suggested EBV reactivation state and one was asymptomatic EBV carrier. In three patients, tissues EBV ISH were done and all were positive to EBER and lytic probe.

\section{MULTIPLE SCLEROTIC FIBROMAS IN AN HIV- POSITIVE PATIENT WITHOUT EVIDENCE OF COWDEN'S DISEASE}

Joseph W. Olivere, Timothy P. Monahan and Terry L. Barrett Department of Dermatology, Division of Dermatopathology, Johns Hopkins Medical Institutions, Baltimore, MD, USA

Sclerotic fibroma is an uncommon benign neoplasm of the skin which may occur sporadically in a solitary form or as multiple lesions as a component of Cowden's disease. Sclerotic fibromas have a distinctive histologic appearance characterized by well-circumscribed, hypocellular dermal nodules of thick, hyalinized collagen bundles arranged in a whorled or storiform pattern with prominent clefts. We present the case of a 52-year-old HIV-positive, African-American female who presented with multiple firm flesh-colored nodules of several year duration on bilateral lower extremities. Biopsies of the lesions demonstrated characteristic features of sclerotic fibroma. Detailed physical examination and extensive systemic work-up failed to reveal evidence of Cowden's disease. To our knowledge, multiple sclerotic fibromas have not been reported in the absence of Cowden's disease, and this is the first reported case of multiple sclerotic fibromas occurring in an HIV-positive patient without evidence of Cowden's disease. 


\section{ECTOPIC MENINGOTHELIAL HAMARTOMA OF THE} SCALP

A.O. Osunkoya, T. Foster and J Pitha

Department of Pathology, University of Oklahoma Health Sciences Center, Oklahoma city, OK, USA

A 7-month-old male infant presented with a 2 month history of a solitary, well circumscribed, $1.2 \mathrm{~cm}$ right parietal scalp lesion. An MRI excluded intracranial extension of the lesion. Grossly the excised lesion was tan-grey, soft to rubbery with focal areas of hemorrhage. On histology, the lesion demonstrated elongated strands of haphazardly arranged fibrous tissue and meningothelial elements with clusters of plump, cuboidal epitheloid cells extending along connective tissue planes in the dermis and subcutaneous tissue. The meningothelial elements were intimately admixed with mature adipose tissue and proliferation of small to medium sized vessels. The overlying epidermis was unremarkable. No mitotic figures or areas of necrosis were identified. The meningothelial cells were positive for Vimentin and Epithelial Membrane Antigen. S-100 protein was negative and CD-34 was positive only in the endothelial cells. The gross, histologic and immunohistochemical features are consistent with ectopic meningothelial hamartoma of the scalp. The meningothelial elements characteristic of this entity are believed to arise from displaced, ectopic meningothelial rests in the scalp and are thus regarded as simply representing a proliferation of the hamartomatous process.

\section{POROKERATOSIS-LIKE CHANGES IN CHONDRODERMATITIS NODULARIS HELICIS:CONSEQUENGE OR COINCIDENCE? \\ R.N. Page, R. King and P.B. Googe \\ Knoxville Dermatopathology Laboratory, University of Tennessee, Graduate School of Medicine, Knoxville, TN, Vanderbilt Medical School, Nashville, TN, USA}

Background: Chondrodermatitis nodularis helicis $(\mathrm{CNH})$ is a condition with known predisposing factors, but of unknown etiology. A variety of opinions exist as to the cause of this condition, without consensus. Design: We reviewed 111 cases of CNH from 103 patients at our laboratory. p53 immunohistochemical staining, which is over expressed in basilar epidermal cells of porokeratosis, was performed in a representative sample of $\mathrm{CNH}$ cases. Histologic changes characteristic of porokeratosis were qualified as a 1) parakeratotic column of keratinocytes 2) an attenuated to absent granular layer, and 3) adjacent or underlying dyskeratosis of keratinocytes, present in columns or aggregates. Results: Porokeratosis-like changes were identified in 77 of 111 cases. p53 staining was noncontributory. Conclusion: The presence of these porokeratosis-like changes in $69 \%$ of cases is intriguing. It is not clear whether or not this could possibly represent a causative mechanism or, perhaps, an otherwise coincident histologic change. The presence of porokeratosis-like changes in $\mathrm{CNH}$ provides a possible hypothesis in relationship to its etiology, at the very least, it could provide some histologic clue as to the presence of this deep dermal process in superficial biopsies.

\section{THROMBOGENIC VASCULOPATHY AND INTERSTITIAL TO DIFFUSE DERMAL NEUTROPHILIC INFLAMMATION AS A HISTOLOGIC MANIFESTATION OF TICK BITE REACTION}

Urvi Pajvani BS, Orazio Basile MD, Neil Toback MD, Priya Swamy

Zeikus, MD and Leslie Robinson-Bostom MD

Rhode Island Hospital, Brown Medical School Department of Dermatology, Providence RI
Ticks are ectoparasites that cause dermatologic disease both directly through physical trauma to the skin, salivary secretions, or remnant body parts, and indirectly through transmission of disease. Lyme disease, Rocky Mountain spotted fever, tularemia, and babesiosis are known tick-transmitted diseases. The histopathology related to a primary tick bite, similar to other arthropod bites, classically consists of a perivascular infiltrate composed of lymphocytes, neutrophils, histiocytes, plasma cells, and eosinophils in varying amounts. We describe five patients with a novel histologic reaction to embedded tick parts., Each case demonstrates a thrombotic vasculopathy consisting of intraluminal eosinophilic deposits that stain strongly with Periodic acid Schiff stain. The adjacent tissue shows dermal necrosis with surrounding interstitial to diffuse dermal neutrophilic inflammation. We postulate that diffuse dermal nutrophilic infiltrates in association with thrombogenic vasculopathy is an unusual histologic picture of tick bite reactions that may be attributable to remnant tick parts.

\section{RESPIRATORY EPITHELIAL ORBITAL GYST: REPORT} OF A CASE AND REVIEW OF THE LITERATURE

$\mathrm{S}$ Pasternak ${ }^{1}$, M Humayun ${ }^{2}$ and $\mathrm{N}$ Walsh ${ }^{1}$

Departments of Pathology ${ }^{1}$ and Ophthalmology ${ }^{2}$,

Capital District Health Authority and Dalhousie University, Halifax, Nova Scotia, Canada.

Orbital cysts include the common epidermoid and dermoid cysts, cysts of conjunctival origin and cysts lined by respiratory-type epithelium. Respiratory epithelial cysts are exceedingly rare and have been reported in common locations for dermoid cysts (superotemporal and superonasal anterior orbit) and in sites atypical for dermoid cysts. Most represent maxillary sinus mucoceles extending into the orbital floor. In some patients, a history of orbital trauma or previous sinus surgery has been implicated in the implantation of the respiratory epithelium in the orbit. Rarely, the respiratory epithelial cyst has been considered to be a choristoma. We report the case of a 72-yearold woman who presented with a cyst in the anterior superotemporal orbit. There was no history of recent or remote trauma. The clinical impression was of a dermoid cyst. Histopathologic examination revealed a cyst lined by non-keratinizing squamous epithelium. A few goblet cells were present and focally, short strips of ciliated mucin secreting columnar cells were identified. The histopathologic findings were those of a respiratory epithelial cyst of the orbit. Given the rarity of this entity, valuable information can be gleaned from critical evaluation and reporting of new cases, in the context of existing data in the literature.

\section{PRIMARY CUTANEOUS EWING'S SARCOMA/ PRIMITIVE NEUROECTODERMAL TUMOR MIMICKING MALIGNANT ECCRINE SPIRADENOMA WITH 22Q12 TRANSLOCATION BY INTERPHASE FLUORESCENT IN SITU HYBRIDIZATION \\ R.M. Patel, J.R. Goldblum, M. Skacel, E. Downs-Kelly, R. Tubbs, C. Embi and W.F. Bergfeld \\ Department of Pathology, Emory University, Atlanta, GA; Divisions of Pathology and Laboratory Medicine and Dermatology, The Cleveland Clinic Foundation, Cleveland, $\mathrm{OH}$}

Gutaneous small blue cell tumors are relatively uncommon neoplasms whose differential includes primary adnexal, neuroendocrine and lymphoproliferative tumors, as well as metastatic lesions. Another entity to be considered in the differential is Ewing's sarcoma (ES)/ primitive neuroectodermal tumor (PNET). We present a 19-year-old female who developed a solitary, subcutaneous mass on her back. 
Histology revealed a poorly differentiated, cellular, nodular neoplasm of small, blue cells containing round to ovoid, vesicular nuclei with indistinct nucleoli and scant eosinophilic cytoplasm. Tumor cells were strongly immunoreactive for antibodies to carcinoembryonic antigen (CEA), Cam 5.2 and CD99. Overall, the lesion resembled an malignant spiradenoma (spiradenocarcinoma). CD99 reactivity prompted analysis with dual-color interphase FISH for translocations involving (22q12), which was positive, a sensitive marker for ES/PNET. Our case demonstrates that a diagnosis of ES/PNET should be considered in the differential of primary cutaneous blue cell tumors. These lesions are difficult to distinguish from other cutaneous small blue cell tumors due to their rarity and because the diagnosis is often not considered at this site.

\section{SQUAMOUS GELL CARCINOMA ASSOCIATED WITH EPIDERMAL NEVUS}

A. Peralta Soler, D.S. Becker, S.B. Peters and N.S. McNutt

Dept. of Dermatopathology and Dermatology, Weill Cornell Univ. New York, NY

We present the case of a 27 year-old female with a linear array of brown keratotic papules with a raised nodule within, located in the right mid upper inner thigh. A biopsy of the nodule showed a superficially invasive squamous cell carcinoma. Subsequent excision of the entire lesion showed an epidermal nevus without residual carcinoma. Squamous cell carcinomas developing within an epidermal nevus constitute an unusual association, mostly occurring in long standing lesions of elderly patients. Other epithelial neoplasms described arising within epidermal nevi include basal cell carcinomas, malignant eccrine tumors and keratoacanthomas. Although our patient did not have an identifiable epidermal nevus syndrome, the presence of a squamous cell carcinoma within an epidermal nevus in a young person suggests increased susceptibility to tumor development. Moreover, chromosomal aberrations have been described in epidermal nevi, including chromosomal breakage and variegated translocation mosaicism. We suggest that the presence of an epidermal nevus should alert the clinician for the potential development of a malignant neoplasm within it, including squamous cell carcinoma, even in the absence of an identifiable hamartoma-neoplasm syndrome.

\section{DIFFERENTIAL EXPRESSION OF JUNB IN CUTANEOUS CD30-POSITIVE DISORDERS}

A.G. Perna, A. Major, J.A. Reed and D. Lopez-Terrada Department of Pathology, Baylor College of Medicine, Houston, Texas, USA

Although some classic differences exist histologically between malignant and benign CD30-positive lymphoproliferative disorders of the skin, the distinction can only be made by correlation with the patient's clinical course. There has been limited investigation into the genetic abnormalities seen in this class of disorders. Cutaneous ALCL has been shown to exhibit increased expression of JunB, but differences in expression of JunB between c-ALCL and benign CD30-positive cutaneous lymphoproliferative disorders have yet to be evaluated. A study to determine the prevalence of JunB coexpression in CD30positive cells of primary cutaneous anaplastic large cell lymphoma and benign CD30-positive disorders of the skin was undertaken. The two c-ALCL cases had an average of $98.3 \%(+/-0.7 \%)$ of CD30positive cells coexpressing JunB and the seven benign CD30-positive disorders exhibited an average of $88.9 \%(+/-7.4 \%)$ of CD30positive cells coexpressing JunB. There was a trend towards lower JunB and CD30 coexpression with benign CD30 lesions compared to cutaneous ALCL, but with our limited sample size, this difference did not achieve statistical significance. Further investigation is needed to more fully evaluate the sensitivity of the expression of JunB and other proteins as markers for clinical behavior in CD-30 positive lymphoproliferative disorders.

\section{COMPOSITE EXTRARENAL RHABDOID TUMOR OF THE SKIN: CYTOLOGY, MORPHOLOGY, ELECTRON MICROSCOPY AND SPECIAL STAIN FINDINGS}

M. Petitt, K. Doeden, A. Harris and T. Bocklage

Department of Pathology, University of New Mexico Health Sciences Center, Albuquerque, NM, USA

We present the findings of a rare and very aggressive primary skin tumor, composite extrarenal rhabdoid tumor (CERT) with features of rhabdomyosarcoma. Clinical history: A 53-year-old woman presented with a $2.5 \mathrm{~cm}$ ulcerated nodule on her right medial calf. On exam, she also exhibited inguinal lymphadenopathy. Pathology: Fine needle aspiration of a lymph node and punch biopsy of the skin nodule disclosed an epithelioid cell morphology with rhabdoid cells, solid growth, and a high mitotic rate (>70 figures/10 HPF). Upon wide excision of the tumor, electron microscopy $(\mathrm{EM})$ and special stains were performed. Since the differential diagnosis was broad, a large number of stains were performed: tumor was positive for vimentin, desmin, muscle specific actin, WT-1, CD56, and CD10, and negative for S-100, melan A, cytokeratins, EMA, CD34, fast myosin, myoglobin, myogenin, myoDl, smooth muscle actin, and NSE. EM revealed rhabdoid cells with perinuclear globules of intermediate filaments corresponding to the positive desmin stain. Summary: The patient was treated with chemotherapy without regression of presumed lung metastases. The CERT is a rare, aggressive tumor that extraordinarily presents as a primary skin tumor. The differential is broad and nomenclature confusing. Extensive ancillary pathology studies are required for appropriate diagnosis.

\section{GRANULOMATOUS LYMPHANGIITIS (MELKERSSON- ROSENTHAL TYPE}

V. Petronic-Rosic, H. Sattar, L. White, A. Laumann, T. Krausz and C.R. Shea

Section of Dermatology and Department of Pathology, The University of Chicago, Chicago, IL, USA

A 33-year-old Caucasian female presented with a ten-year history of flushing and facial swelling partially responsive to anti-inflammatory and anti-microbial therapies. Progressive edema of the right upper eyelid was recently treated by blepharoplasty. The blepharoplasty specimen had a dense mononuclear inflammatory infiltrate and diffuse non-caseating granulomas composed of epithelioid and multinucleated histiocytes. The granulomas appeared both peri-and intralymphatic with prominent molding. Special stains and polarization studies were negative. To confirm the intralymphatic location of these granulomas we performed immunohistochemical staining with Factor VIII and D2-40. D2-40 is a new monoclonal antibody that reacts with an O-linked sialoglycoprotein found on lymphatic endothelium, fetal testis, and on the surface of testicular germ cell tumors. In recent studies, clone D2-40 has shown staining in lymphatic channel endothelium but not in adjacent capillary. Factor VIII antibody highlighted both lymphatic and blood vessel endothelium, while D2-40 stained only the lymphatic endothelium and confirmed the intra-lymphatic location of granulomas. The patient was diagnosed with granulomatous lymphangiitis of the Melkersson-Rosenthal type (orofacial granulomatosis). Granulomatous lymphangiitis is characteristic but not completely diagnostic of the MelkerssonRosenthal syndrome. We present the complex differential diagnosis 
of intralymphatic granulomas and the use of a new monoclonal antibody helpful in verifying their location.

\section{MULTIFOCAL INFANTILE FIBROMATOSIS \\ T. Pham, J. Madden, M.A. Selim and C. Herman \\ Department of Pathology, Duke University Medical Center, Durham, NC, USA}

Infantile fibromatosis (IF) is an uncommon childhood disorder characterized by solitary or muticentric tumors involving skeletal muscle, skin, bones, or viscera. There is a wide morphologic spectrum reflecting various stages of fibroblastic differentiation. We present the clinical and histopathologic findings of a rare case of IF. A seven month old female presented with firm masses over the right elbow $(2 \times 3 \mathrm{~cm}$. $)$ and right distal clavicle $(3 \times 4 \mathrm{~cm}$.). Magnetic resonance imaging demonstrated increased $\mathrm{T} 2$ signal within the soft tissues of the right axilla, supraclavicular region, and distal humerus. However, there was no evidence for bony involvement. Incisional biopsy of the supraclavicular mass showed a white fibrous grossly appearing tumor. Histologic examination revealed clusters of adipocytes and infiltration of skeletal muscle fibers by a moderately cellular proliferation of ovoid within a fibrous background. There was no atypia, necrosis, or increased mitotic activity. The tumor cells showed diffuse immunoreactivity for vimentin and weak focal positivity for smooth muscle actin. No reactivity was seen with S100. In addition, none of the tumor cells exhibited nuclear estrogen receptor expression by image cytometry. This case is reported in an effort to better define the clinical and histopathologic features of infantile fibromatosis.

\section{P-ANCA POSITIVE COCAINE ASSOCIATED VASGULITIS: A CASE REPORT}

T. Pham, C. Heinly, C. Herman and M. A. Selim

Department of Pathology, Duke University Medical Center, Department of Dermatology, Veteran Administration Medical Center, Durham, NC, USA

Antineutrophilic cytoplasmic antibodies (ANCA) are classically associated with primary vasculitic syndromes, including ChurgStrauss syndrome, microscopic polyangiitis, and Wegeners granulomatosis. Recently, drug induced ANCA positive vasculitis has been reported, most frequently in association with propylthiouracil in Grave's disease. Cocaine use is known to be associated with pulmonary and systemic eosinophilia as well as various vascular pathology, including vasospasm, atherosclerosis, and vasculitis. We report a case of cocaine associated perinuclear antineutrophilic cytoplasmic antibody (p-ANCA) positive vasculitis. A 46-year-old man with a history of crack cocaine abuse presented with arthralgia, lower extremity edema, and multiple purpuric, tender papules and plaques over the hands and lower extremities. Peripheral blood studies revealed hypereosinophilia, and serologic tests demonstrated a p-ANCA titer of 1:2560. A skin biopsy demonstrated injured small dermal vessels with a perivascular infiltrate of neutrophils, karyorrhectic debris, erythrocytes, and abundant eosinophils. His symptoms and eosinophilia resolved with cessation of cocaine use and intravenous dexamethasone. Following a 2 year period of reported abstinence, the patient relapsed on cocaine and presented with similar clinical, serologic, and histologic findings. Although p-ANCA has been reported in patients with cocaine associated midline destructive lesions, to our knowledge, this is the first report of cocaine associated p-ANCA positive systemic vasculitis.

\author{
A CASE REPORT OF UNUSUAL MULTIPLE MINUTE \\ DIGITATE HYPERKERATOSIS \\ D. Pierson ${ }^{1}$, K. Seilstad ${ }^{2}$ and R. Rogers ${ }^{2}$ \\ Departments of Pathology ${ }^{1}$ and Dermatology ${ }^{2}$, \\ Mayo Clinic, Rochester, MN, USA
}

We report a 43-year-old woman, in otherwise excellent health, who presented with a 4 year history of an asymptomatic, papular condition described as 'tiny little spines' involving the skin. On examination hundreds of discrete, palpable $1-\mathrm{mm}$ projections were present on the upper torso and arms. Histopathology showed a small focus of acanthotic epidermis forming a small spire. Overlying the subtle epidermal elevation was a column of compact hyperkeratosis with focal parakeratosis. Epidermal atrophy and dermal lymphocytic inflammation, characteristic of Flegel's disease, were not observed. No features of keratosis pilaris were noted. A diagnosis of multiple minute digitate hyperkeratoses was rendered. Relatively rare multiple minute digitate hyperkeratoses is a non-follicular disorder of keratinization of unclear etiology. Previously ascribed names include, but are not limited to, disseminated spiked hyperkeratosis and minute aggregate keratoses. A classification scheme with sporadic, post-inflammatory (recently challenged as another entity) and familial (autosomal dominant) types has been proposed. Other classifications are based on topography and histology or age of onset: early (20's and 30's) and late (40's-50's). Paraneoplastic syndromes have been associated with late onset type. In summary, we report a sporadic case of late-onset multiple minute digitate hyperkeratosis, an unusual and relatively rare disorder.

\section{TRENDS IN CLARK LEVEL AND BRESLOW THICKNESS OF INVASIVE CUTANEOUS MALIGNANT MELANOMA OVER A 50-YEAR PERIOD IN OLMSTED COUNTY/ ROCHESTER, MN}

D. Pierson ${ }^{1}$, A. Subtil ${ }^{2}$, M. Pittelkow ${ }^{3}$, G. Scott ${ }^{4}$, R. Weenig ${ }^{5}$, C. Vachon ${ }^{6}$

Departments of Pathology ${ }^{1}$, Dermatology ${ }^{2,3,5}$,

Division of Biostatistics ${ }^{4}$

and Epidemiology ${ }^{6}$,

Mayo Clinic, Rochester, MN, USA

Incidence of invasive melanoma is reported to be steadily increasing worldwide. Whether due to an actual increase in disease, or increased surveillance and changes in diagnostic criteria, or both is unknown. The purpose of this study was to analyze Clark Level and Breslow thickness trends in a population-based sample over five decades. All cases of invasive melanoma, a total of 200, diagnosed from 1985-1999 were identified using the Rochester Epidemiology Project that incorporates medical-surgical index information for all residents of Rochester/Olmsted County. These findings updated two previous studies of invasive melanoma from 1950-1985, resulting in 296 total cases over the 1950-1999 period. The incidence of Clark level II lesions increased from $0 \%$ in $1950-1959$ to $51 \%$ in $1990-1999$, while Clark level V lesions decreased from $38 \%$ to $4 \%$. Moreover, the incidence of lesions with Breslow $4 \mathrm{~mm}$ decreased from $25 \%$ to $7 \%$. These variations were found to be statistically significant $(\mathrm{p}=<0.01)$. The decreases in Clark level and Breslow thickness seen in this population-based sample over the last 5 decades suggests that a significant component of increased invasive melanoma incidence may be explained by increased surveillance and earlier detection. 


\section{PYODERMA GANGRENOSUM-LIKE CUTANEOUS} T-CELL LYMPHOMA

M. Piliang, J. McDonnell, R. Tuthill and J. Libecco

Departments of Dermatology and Dermatopathology, Cleveland Clinic Foundation, Cleveland, OH, USA

A 25-year-old man presented with a large, undermined, violaceous ulcer with hemorrhagic crust on his nose for 1 year. It began as a "pimple". He had similar lesions on his scalp, abdomen and groin. His sister has Crohn's and his mother has rheumatoid arthritis. Screening blood tests and CT scans revealed no underlying disease. The clinical impression was pyoderma gangrenosum. Biopsies from the nose and abdomen revealed dense superficial and mid-dermal perivascular lymphocytic infiltrate composed of enlarged, atypical lymphocytes with focal epidermotropism and epidermal necrosis. Immunohistochemistry revealed the infiltrate was primarily CD3 positive T-cells without deficiency of CD4, CD7 or CD8. CD30, TIA and Granzyme were not detected. A T-cell receptor gamma gene rearrangement was detected. Flow cytometry was negative. This is a case of pyoderma gangrenosum-like cutaneous T-cell lymphoma, a rare form of cutaneous lymphoma that clinically resembles PG, but histologically and immunohistochemically is cutaneous T-cell lymphoma. Although rare, PG-like ulcers should be added to the list of atypical manifestations of CTCL. This case highlights the need to evaluate all atypical lymphocytic infiltrates thoroughly with immunohistochemistry and gene rearrangement. The diagnosis must be made with caution as proliferative clones of lymphocytes can be found in true PG.

\section{THE PATHOLOGY OF ADULTERATED INJECTED COSMETIC FILLER MATERIALS}

M. Pulitzer, MD, J. Tan, MD, PhD, J. Levitt, MD and R. Phelps, MD

Department of Pathology, Mount Sinai School of Medicine, New York, NY, USA

We report five cases of illicit injections of filler-substances for cosmetic purposes in non-medical clinics. The first two patients, a mother and 30-year-old daughter, presented with skin lesions in both legs after calf-augmentation by paraffin injection 4 years prior. One week later calf hyperpigmentation and induration developed, with progression up the legs, and eventual purulent draining ulceration (daughter). The third patient, a 34-year-old female, presented with pink-brown macules on both buttocks after receiving silicone injections 9 months earlier. The fourth patient, a 31-year-old female, developed skin lesions on the buttocks after silicone injections. The fifth patient, a 43-year-old female, developed sclerodermoid plaques on the buttocks after silicone injection. The first two biopsies showed large spaces in tissue, calcinosis cutis, dermal sclerosis, granulation tissue, and lymphedema. The third biopsy revealed foreign body reaction to nonpolarizing material, the fourth lipogranulomatous reaction, and the fifth microvacuolar change with scar. Biopsies post-injection of cosmetic filler materials typically show macrovacuolar "swiss cheese" changes, and later microvacuolar changes with considerable granulomatous reaction. The reactions here were clinically severe, and may reflect adulteration of injected materials. We believe these cases augur a public health epidemic given a proliferation of non-medical cosmetic "mills" which offer illicit cosmetic procedures.

\section{GLOMUS COCGYGEUM: REPORT OF A CASE}

A. Rahemtullah, K. Szyfelbein, A. Zembowicz

Dermatopathology Unit, Department of Pathology, Massachusetts General Hospital and Massachusetts Eye and Ear Infirmary, Harvard Medical School, Boston, MA, USA
The glomus coccygeum is a vestigial structure related to the canals of Sucquet-Hoyer, an arteriovenous anastomosis surrounded by glomus cells derived from modified smooth muscle and involved in thermoregulation. It is an incidental finding in specimens from the sacral area and may represent a diagnostic challenge to the unaware observer. We present a case of a glomus coccygeum, presenting as a $1.5 \mathrm{~mm}$ structure adjacent to a typical pilonidal cyst excised from a 7-month-old boy, that was the subject of a second opinion consultation. The lesion showed small to medium sized clusters of predominantly epithelioid cells with moderate amounts of clear to eosinophilic cytoplasm, intercellular borders and plump, round nuclei with fine chromatin. These cells were closely associated with small vascular channels and nerves. Immunohistochemistry revealed that the epithelioid cells expressed vimentin, muscle-specific actin, neuron-specific enolase, and S-100 protein, were weakly positive for smooth muscle actin, and negative for desmin, synaptophysin and chromogranin. The endothelial cells of the vascular channels were antibody CD31 positive. Recognition of the histological features of glomus coccygeum is important to avoid confusion with glomus tumor and neural or smooth muscle neoplasms in the sacral area.

\section{PRIMARY CUTANEOUS CARGINOSARCOMA (PCCS0} AKA METAPLASTIC CARCINOMA)

Ramin Ram, Payam Saadat and Manju Vadmal

Department of Pathology and Dermatology, University of Southern California, Los Angeles, CA, USA

We describe a case of PCCS in a 73 year-old male who presented with a rapidly growing tumor of 6 months duration on the right ear. Clinical examination revealed a $5 \times 4 \times 4$-cm ulcerated, crusted, exophytic tumor on the superior aspect of the helix. Histologically, the mass revealed a biphasic tumor with malignant epithelial and mesenchymal components. The epithelial component consisted of a few foci of basal cell carcinoma (BCG). The majority of the tumor was composed of osteogenic sarcoma and malignant fibrous histiocytoma $(\mathrm{MFH})$. An intricate transition of BCC into sarcoma was noted in a few foci. Atypical mitosis and necrosis were common. The BCG and tumor giant cells in the $\mathrm{MFH}$ area showed positive immunoreaction for cytokeratin and CD68 respectively. The tumor was negative for S100 protein, HMB-45, muscle actins, chromogranin, and synaptophysin. In conclusion, a PCCS growing predominantly as osteosarcoma and MFH is reported. Although the exact histogenesis of PCCS is unknown, primitive mesenchymal cells of the dermis, surrounding the follicular units, are capable of pluripotent differentiation and are likely the source of origin of the sarcoma. The known aggressive biological behavior warrants wide excision and given the rare reports of metastasis and death, regular follow-up is required

\section{LAMININ-5 EXPRESSION IN MELANOCYTIC LESIONS OF THE SKIN}

J.A. Ramirez, L.K. Diaz and J. Guitart

Departments of Dermatology and Pathology, Northwestern University Feinberg School of Medicine, Chicago, IL, USA

Background: Laminin-5 is a basement membrane constituent that functions as an anchoring filament to integrin-derived hemidesmosomes, and has been detected at the invasive front (tumor-stromal interface) in many types of carcinomas. Our goal was to analyze laminin-5 expression in melanocytic lesions and evaluate its role in tumor progression. Design: Immunohistochemical staining for laminin-5 was performed on 101 cutaneous melanocytic lesions including 41 nevi, 31 primary melanomas, and 29 metastatic melanomas. A standard immunoperoxidase technique $(\mathrm{ABC})$ was used with $\mathrm{DAB}$ 
chromagen and a primary monoclonal antibody to the laminin-5 gamma2 chain (clone D4B5, 1:50, Chemicon International, Temecula, CA). Any degree of staining in melanoma cells or at the tumorstromal interface was considered positive. Results: Positive staining for laminin-5 was observed in three of the primary melanomas $(10.7 \%)$, three of the metastatic melanomas $(10.3 \%)$, and none of the nevi. In primary melanomas, staining was observed predominately at the tumor-stromal interface. In metastatic lesions, staining was observed around individual melanoma cells and melanoma cell nests.Conclusion: Expression of laminin-5 was detected in a small percentage of primary and metastatic melanomas and in none of the nevi studied. Laminin-5 expression does not appear to be essential for the development of melanomas or their metastases.

\section{GYCLIN D1 EXPRESSION IN MELANOGYTIC LESIONS OF THE SKIN}

J.A. Ramirez, L.K. Diaz and J. Guitart

Departments of Dermatology and Pathology, Northwestern

University Feinberg School of Medicine, Chicago, Il, USA

Background: Progression through the cell cycle is controlled by cyclins, cyclin-dependent kinases and cyclin-dependent kinase-inhibitory proteins. The role of cyclin D1 in the development, progression and prognosis of melanomas is controversial. The goal of this study is to evaluate the role of cyclin D1 in benign and malignant melanocytic lesions of the skin. Methods: A total of 101 melanocytic lesions of the skin including compound nevi (21), intradermal nevi (18), melanoma in situ (3), primary invasive melanoma (30), and metastatic melanoma (29) were evaluated for cyclin D1 overexpression by immunohistochemistry. The following tiered system was used for scoring: $0 \%$ of cells with nuclear staining (score 0), 1-19\% nuclear staining (score 1), $20-49 \%$ nuclear staining (score 2), and $50 \%$ or greater nuclear staining (score 3). Results: The average score for primary melanomas was significantly higher compared to nevi $(\mathrm{p}=0.0046)$, and for in situ melanomas compared to primary invasive melanomas $(p=0.011)$. There was slightly higher level of expression in compound nevi versus intradermal nevi. Conclusion: Our study indicates that cyclin D1 expression is increased in malignant compared to benign melanocytic lesions. Further studies are needed to ascertain the biological role of cyclin D1 in melanocytic lesions of the skin.

\section{WILL SKIN PATHOLOGISTS EVER BE REPLACED BY NONINVASIVE DIAGNOSTIC TOOLS (COMPUTERS)? B. Rao, B. Katz, N. Wei and P. Park \\ Robert Wood Johnson Medical School, Division of Dermatology, New Brunswick, NJ, USA}

Title: Will skin pathologists ever be replaced by noninvasive diagnostic tools (computers)? Intro: There are now many new noninvasive tools being developed to diagnose skin cancer, specifically melanocytic neoplasms. Whether these tools will ever be able to diagnose as well as a pathologist remains to be seen. In this paper, we will review most of the newly developed tools and discuss their specificity, sensitivity and diagnostic accuracy. Method: We reviewed the literature on currently available noninvasive diagnostic tools to find the published sensitivity and specificity rates of these tools. Tools without published information on their diagnostic accuracy were not included. We tabulated the accuracy data on the remaining tools and evaluated their relative diagnostic accuracy. Results: Melafind was found to have a specificity of $68-85 \%$ and a sensitivity of $95 \%-100 \%$. Siascope was found to have a specificity of $82.7 \%$ and a sensitivity of $80.1 \%$. Solarscan was found to have a specificity of $83 \%$ and a sensitivity of $100 \%$. Comments: Non-invasive tools may be helpful in managing melanocytic neoplasms in patients with numerous lesions or with lesions in a cosmetically important site i.e. face. At present these tools cannot replace the value of a biopsy, microscopically reviewed by a pathologist.

\section{MYCOSIS FUNGOIDES MIMICKING LICHEN PLANUS \\ Babar K. Rao, Janet Merino and Irfan Rahman}

Introduction: To report an uncommon case of mycosis fungoides in a 62-year-old female. Case presentation: A 62-year-old Black female presented to our clinic with a wide spread of hyper-pigmented erythematous grayish plaques on trunk, extremities and face. There were no associated systemic illnesses and laboratory work was otherwise within normal range. Two skin biopsies were taken from the trunk and extremity. Both of the biopsies reveal similar features of coexisting like lichen planus and mycosis fungoides. There was interface dermatitis, vacuolar degeneration, prominent colloid body formation and lichenoid lymphonocytic inflammation through out the dermis. Epidermis was atrophic with overlying hyper and parakeratosis. There were many areas of epidermotrophism with collections of atypical lymphocytes. There was also marked pigmentory incontinence in the dermis. Comment: We present an uncommon case of mycosis fungoides with changes of lichen planus. It is important familiarise oneself with uncommon presentations of mycosis fungoides. It may be worthwhile to look for mycosis fungoides, while reviewing a classical case of lichen planus with clinical presentation of lichen planus.

\section{GUTANEOUS RHIZOPUS IN AN IMMUNOSUPPRESSED PATIENT}

S. Ravis, S. Frankel and G. Elgart

Department of Dermatology, University of Miami, Miami, FL, USA

The patient is an eight-year-old white male with a prior history of a small bowel transplant secondary to gastroschisis and a history of chronic rejection. He was admitted to the hospital to rule out rejection of his second small bowel transplant. During the admission he developed painful erythematous plaques with necrosis and hemorrhagic bullae on his left knee and left neck. Dermatology was consulted and biopsies and tissue cultures were obtained. On H\&E there were multiple thick walled non-septate hyphae and the GMS stain showed right angle irregular branching hyphae with an affinity for blood vessels. A further work-up for disseminated disease was negative. The patient was diagnosed with cutaneous mucormycosis. The patient underwent extensive surgical debridement and was started on Ambisone and treated for six months with resolution. Primary cutaneous mucormycosis is a rare opportunistic deep fungal infection. It is the most acute and fatal of all fungal infections. There are multiple associated conditions including immunosuppression. This disease can be controlled with early diagnosis and a combination treatment plan. There are only a few reported cases in the pediatric population.

\section{DISSEMINATED ACANTHAMOEBIASIS}

A. Readinger, M Blumberg, Z. Wang, M Colome-Grimmer, J. Aronson and E. Kelly

Department of Dermatology, University of Texas Medical Branch, Galveston, TX, USA

A 60-year-old male with a history of pulmonary fibrosis and lung transplant presented to clinic with a three week history of cutaneous eruption on the extremities associated with nausea and fatigue. His medications included prednisone and azathioprine. Exam revealed the patient was afebrile with multiple perforating nodules and 
subcutaneous nodules in a sporotrichoid pattern over the extremities. Tissue was sent for histopathological evaluation, and culture for bacteria, acid-fast bacteria, and deep fungal organisms was performed. Histopathology revealed necrotizing panniculitis, but no organisms were found, and tissue cultures were negative. Due to choreoathetoid movements of the right extremities and persistence of cutaneous nodules, the patient was hospitalized and an extensive search for infectious etiology of his condition undertaken. Empiric antibacterial and antifungal therapy was given, but he progressed to multiorgan failure and expired. Autopsy revealed rare amoebic trophozoite forms in the skin in addition to cysts in the lung and brain. Cause of death was determined to be disseminated Acanthamoebiasis. Review of initial skin biopsy showed rare trophozoites. Differing from Entamoeba, Acanthamoeba is a rare but important emerging pathogen. Histopathological diagnosis of Acanthamoeba rests on high index of suspicion in cases of suspected infectious etiology.

\section{INCONTINENTIA PIGMENTI IN A MALE WITH MOSAIC KLINEFELTER SYNDROME: THE ROLE OF FISH ANALYSIS \\ J. Reutter, J. Goldstein, N. Prose, M. McDonald and M. Selim Departments of Pathology, Medical Genetics, Dermatology, and Pediatrics, Duke University Medical Genter, Durham, NG, USA}

Incontinentia Pigmenti (IP) is an X-linked dominant genodermatosis with cutaneous, skeletal, ocular, neurological, and dental abnormalities. It has been mapped to chromosome Xq28 encoding the NF-kB essential modulator (NEMO). NEMO regulates many genes, such as those that prevent apoptosis. IP is usually lethal in males. However, patients with Klinefelter syndrome (47, XXY) are able to survive due to one normal $\mathrm{X}$ chromosome. We report a case of a male who presented after birth with multiple vesicles in a linear distribution on the calf progressing to persistent hyperpigmentation. At one month of age a punch biopsy showed verrucous hyperplasia with mild hypergranulosis, necrotic dyskeratinocytes and a lichenoid infiltrate composed of lymphocytes, eosinophils and melanophages. A diagnosis of IP was rendered. No additional physical findings were noted. The patient was evaluated for Klinefelter syndrome. While his karyotype was $46, \mathrm{XY}$ by conventional cytogenetics, a dual color interphase FISH detected 92.5\% 46, XY and 6.5\% 47, XXY. Our patient's unique genetic array allowed a mechanism to escape and otherwise fatal disorder. This case illustrates the potential utility of using FISH analysis to detect Klinefelter syndrome in a male patient with IP and normal conventional chromosomal analysis.

\section{THE ROLE OF KI-67 IN PREDICTING PROGNOSIS OF MALIGNANT MELANOMA OF THE VULVA}

Reutter JC, Hoang M, Vollmer RT, Groben PA, Thomas DB, and Selim MA

Departments of Pathology, University of Texas Southwestern, Dallas, TX; University of North Carolina, Chapel Hill, NC; and Duke University Medical Center, Durham, NC

Background: Malignant melanoma of the vulva (MMV) accounts for $2 \%$ of all melanomas in women with 5 -year survival rate of $47 \%$. Lymph node involvement predicts survival in these patients. Ki-67 expression has been correlated with lymph node status in cutaneous melanomas. The aim of this study was to evaluate whether Ki-67 correlates with lymph node metastasis in MMV. Methods: Histomorphology and clinical information of $25 \mathrm{MMV}$ were reviewed. The database was divided in MMV with metastasis $(\mathrm{M}+)$ and without lymph node metastasis (M-). A MMV subset was stained for Ki-67 and scored as a percentage (7 patients $\mathrm{M}+$ and 9 patients $\mathrm{M}-$ ). Results: The age range was 36-88 years-old (mean 63 years-old). The tumor thickness mean was $3.14 \mathrm{~mm}$. The mean $\mathrm{Ki}-67$ expression was $23 \%$ for Mand $42.5 \%$ for $\mathrm{M}+(\mathrm{p}=0.07)$. Correlation of tumor thickness and $\mathrm{Ki}-$ 67 expression also has a p value of 0.07 . Logistic regression analysis was obtained.Conclusions: This study shows that $\mathrm{Ki}-67$ expression is associated with lymph node metastasis in MMV. However, when Ki-67 expression is compared to a classic prognostic indicator such as tumor thickness, it appears to have little or no additional predictive value. Larger studies are needed to confirm these results.

\section{AKT/PKB AND MELANOCYTIC PROLIFERATIONS}

Jason B. Robbins M.D., Alan S. Boyd M.D., Darrel L. Ellis M.D., Lillian Nanney Ph.D. and Ann Richmond Ph.D.

Distinguishing among the various melanocytic proliferations remains one of the biggest challenges in dermatopathology. New insights into tumor proliferation may lead to the discovery of helpful immunohistochemical markers. Akt/PKB is a serine/threonine kinase which is a core component of the PI3K signalling pathway. Activation of Akt is seen in many human cancers. This study evaluated Akt activation (phospho-Akt) by immunohistochemistry on 18 intradermal nevi, 8 dysplastic nevi, 9 Spitz nevi, and 27 melanomas. Immunostaining was graded 0 (no staining), 1 (slightly positive), 2 (moderately positive), and 3 (highly positive). The mean immunostaining scores were as follows: intradermal nevi 1.16, dysplastic nevi 1.25, Spitz nevi 2.11, and melanomas 2.15. Spitz nevi exhibited significant pAkt immunoreactivity. Increased pAkt immunoreactivity is seen in melanomas and Spitz nevi as compared to benign intradermal nevi and dysplastic nevi.

\section{THE FIRST KNOWN CASE OF SECONDAY SYPHILIS IN A HEART TRANSPLANT RECIPIENT WITH AN EXTRAORDINARY NUMBER OF SPIROCHETES FOUND ON SKIN BIOPSY}

A. Rubin, M. Grossman and D. Silvers

Department of Dermatology, Columbia University College of Physicians and Surgeons, New York, NY, USA

A sixty-nine-year-old male patient, without a significant prior medical history, presented with a rapidly enlarging, $2 \mathrm{~cm}$ non-tender nodule on the right lower eyelid. The lesion persisted in spite of an incision and drainage. Following an excision, the histology revealed a diffuse infiltrate of atypical mononuclear cells within the dermis. The cells were large, monomorphic, with irregular to convoluted nuclei, prominent nucleoli, amphophilic cytoplasm. They were very mitotically active. Immunohistochemical stains for CD45, CD3, and CD30 were strongly positive. Pancytokeratin, CD20, and ALK (Anaplastic Lymphoma Kinase fusion protein) were negative. The histopathologic diagnosis was CD30 (Ki-l) positive anaplastic large cell lymphoma. The margin was positive for involvement by lymphoma. The lesion was re-excised with no evidence of residual involvement by lymphoma. A primary cutaneous anaplastic large cell lymphoma was favored over systemic involvement based on the clinical presentation of a single eyelid nodule and ALK negativity. He was referred to a hematology-oncologist for further management. Anaplastic large cell lymphoma is a distinctive type of malignant lymphoma with a relatively favorable prognosis. It frequently involves the skin, however, to the best of our knowledge, presentation of this lymphoma type as a single lesion on the eyelid is extremely rare.

\section{CHORDOMA CUTIS: AN UNUSUAL MALIGNANT} TUMOR METASTATIC TO SKIN

A. Rubin, J. Bagel and G. Niedt

Department of Dermatology, Columbia University College of Physicians and Surgeons, New York, NY USA 
A seventy-year-old woman developed back pain associated with a sacral mass seven years prior to presentation. Biopsy of the sacral mass showed chordoma. The patient underwent two surgical resections because of recurrent disease. She presented after developing a firm, non-tender skin nodule on the left back. Biopsy of the lesion showed a dermal neoplasm composed of nests of cells in a mucinous stroma. There was moderate cytologic atypia of the cells, and occasional mitotic figures were seen. Physaliphorous cells were present. The immunostains S-100, cytokeratin, and EMA were all positive. A diagnosis of metastatic chordoma to the skin or chordoma cutis was made. The patient died 15 months later from complications of metastatic disease. Chordomas are rare, representing only $1-4 \%$ of malignant bone tumors, which most often occur in the sacral area. Metastasis to the skin is unusual. In one study, 207 cases of chordoma were evaluated, and only 19 had skin involvement. Chordoma cutis has been described as nodules which are firm, blue domed, cysticappearing, erythematous, translucent, flesh-colored, and reddish brown. Cutaneous metastasis of chordoma has been reported to the nose, chest, back, thigh, shoulders, left arm, face, neck, and scalp.

\section{BULLS-EYE CUTANEOUS INFARCT OF ZYGOMYCOSIS}

A. Rubin, M. Grossman and A. Rabinowitz

Department of Dermatology, Columbia University College of

Physicians and Surgeons, New York, NY USA

A seventy-year-old woman with a history of chronic lymphocytic leukemia was admitted to the hospital for change in mental status. She had a splenectomy five years prior to admission. The white blood cell count was 154,000 cells/microliter with 95\% lymphocytes and 4\% neutrophils. Blood cultures grew Salmonella enteritidis and Candida krusei. She developed a wrist lesion at the site of a previous arterial line. The lesion had a striking resemblance to a bulls-eye, with three distinct concentric zones. A bedside touch preparation rapidly processed with Periodic Acid Schiff staining demonstrated a single intact aseptate broad hypha. Punch biopsy specimens demonstrated broad, aseptate hyphae throughout the dermis and subcutis invading both blood vessels and interstitum. A skin biopsy specimen sent for fungal culture grew Rhizopus arrhizus. Cutaneous zygomycosis is a rare, devastating disease that can occur in both immunocompromised and immunocompetent hosts. This case illustrates a distinct morphology, which we have coined the "bulls-eye infarct of cutaneous zygomycosis". Recognition of this unique appearance allows a rapid bedside diagnosis of this life threatening infection. To our knowledge, this morphology has been reported only twice in the medical literature.

\section{TINCTORIAL PROPERTIES OF ZYGOMYCOSIS IN CUTANEOUS BIOPSY SPECIMENS}

A. Rubin and D. Silvers

Department of Dermatology, Columbia University College of Physicians and Surgeons, New York, NY, USA

It is a little known fact that the organisms causing Zygomycosis are often better visualized with routine Hematoxylin and Eosin ( $\mathrm{H}$ and $\mathrm{E})$ staining than Periodic Acid Schiff (PAS) staining. Experienced dermatopathologists, when evaluating histologic samples suspected of harboring deep fungal infection often rely more heavily on PAS staining to detect fungi. The diagnosis of Zygomycosis may be delayed or missed entirely if sufficient attention is not devoted to the $\mathrm{H}$ and $\mathrm{E}$ stained specimen. A review of multiple dermatopathology textbooks shows there is no universal agreement on the usefulness of routine $\mathrm{H}$ and $\mathrm{E}$ staining versus use of special stains for the detection of Zygomycosis. Grocott's Methanamine Silver (GMS) staining can give false negative results if background staining of reticulum fibers is enhanced. This can occur because of overexposure in silver solution, excessive heat during processing, or use of incorrectly titrated solutions. Three consecutive culture proven cases of cutaneous Zygomycosis infection were evaluated. In each case, organisms were clearly visualized on routine $\mathrm{H}$ and $\mathrm{E}$ sections while PAS staining was variable. Examples of false negative GMS staining are also shown. Recognition of these staining properties can help dermatopathologists better detect the agents of Zygomycosis.

\section{HYPOMELANOSIS OF ITO ASSOCIATED WITH PRECOCIOUS PUBERTY}

B. M. Rutland and M. G. Horenstein

Department of Pathology, University of South Alabama, Mobile, AL, USA

Background: There are only two reported cases of hypomelanosis of Ito $(\mathrm{HI})$ associated with precocious puberty. Here we report a third case, and the first one to be autopsied. Design: A 5-year-old female with $\mathrm{HI}$, intractable seizures and mental retardation presented with gray vaginal discharge and bacterial vaginosis. The patient subsequently died from neurologic complications and was autopsied. Results: Post-mortem examination revealed pigmentary mosaicism following the lines of Blaschko and advanced development of vulvar labia, pubic hair, and breast buds. Other significant autopsy findings included conical teeth, patchy alopecia, macrosomia (body weight and height above 95th percentile), and macrocephaly with megalencephaly (brain $1850 \mathrm{~g}$ ). The uterus and ovaries were unremarkable. Epidermis had decreased melanocytes in hypopigmented areas. Skin fibroblast karyotype was normal. Conclusion: The two previous case reports indicate that the mechanism for precocious puberty in $\mathrm{HI}$ may be either biochemical, as in McCune-Albright Syndrome, or independent of GnRH. The mechanism of the precocious puberty in our case is most likely central.

\section{INCREASING EXPRESSION OF THE RETINOIC X REGEPTOR-B DURING MALIGNANT MELANOMA PROGRESSION \\ S.J. McAlhany ${ }^{1}$ and J.A. Reed ${ }^{2}$}

Medical Scientist Training Program ${ }^{1}$ and Department of Pathology ${ }^{2}$ Baylor College of Medicine, Houston, TX.

Retinoic X receptor-b (RXR-b) is a heterodimerization partner for vitamin D receptor (VDR). 1,25-dihydroxyvitamin D3 activation of VDR leads to growth inhibition in numerous cell lines, including some melanoma lines. Evaluation of VDR and RXR-b expression in vivo in melanocytic neoplasms will increase our understanding of this pathways potential role in growth control. Previous studies in our laboratory showed decreased VDR expression in superficially invasive melanoma, and progressive loss of expression in deeply invasive melanomas and metastatic melanomas (MET). We next sought to evaluate RXR-b expression. Twenty-eight melanocytic neoplasms including 8 melanomas in situ (MIS), 9 primary invasive melanomas (PIM), and 11 MET were evaluated for RXR-b expression by immunohistochemistry. Nuclear labeling was assessed as $0(0 \%), 1+(<5 \%)$, $2+(>5 \%$ but $<50 \%)$, or $3+(>=50 \%)$. A significant increase in RXR-b expression from low $(0-1+)$ to high $(>1+)$ was found when comparing MIS to PIM and MET (chi2 p < 0.05). These data suggest: 1) potential loss of 1,25-dihydroxyvitamin D3 induced growth inhibition during melanoma progression may be due to decreased VDR expression without concomitant loss of RXR-b; and 2) increased RXR-b expression during melanoma progression may offer selective advantage through alternative signaling pathways. 
MALIGNANT MYOEPITHELIOMA OF THE SOLE

Mika Sakaki, Mitsuyoshi Hirokawa, Shingo Wakatsuki, Eri Yoshida and Sano Toshiaki

76-year-old man presented with a hen's egg-sized elevated nodule in his left sole in 4/04. He had been hospitalized because of icterus since 3/04. Although cancer of the pancreatic head had been strongly suspected on the imaging, he had refused surgical operation. No metastasis of the cancer had been found on the imaging. A dermatologist who biopsied the nodule in his left plantar recognized the swelling of inguinal lymph nodes on the same side. Histologically medium sized oval cells formed solid nests in the dermis, rendering them lobulated appearance. Some of the nests showed myxoid change in the stroma where tumor cells often showed cord-like arrangement. The border with surrounding tissue was relatively clear. The cells had round, and often eccentrically located nuclei with one or two small nucleoli and moderate to scarce amount of eosinophilic cytoplasm. Mitosis was readily identifiable; >50/10HPF. Necrosis was not found. Immunohistochemically tumor cells were reactive for $\mathrm{AE} 1 / \mathrm{AE} 3$, vimentin and $\mathrm{S}-100$ protein and negative for ??SMA, CK14, EMA, HMB 45 and Melan A. MIB-1 labeling index was over $50 \%$. The lesion was diagnosed as malignant myoepithelioma. The positivity for S-100 protein seen in this case necessitated us to differentiate it from melanoma.

\section{SEPTIC VASCULITIS FROM FEMORAL ARTERY CATHETERIZATION \\ F. Saporito, C. Stetson and R. Hope \\ Department of Dermatology, Texas Tech Health Sciences Center, Lubbock, TX, USA}

A 77-year-old white male presented to an outpatient clinic with painful, purpuric macules and papules on his right foot. The patient had recently undergone cardiac catheterization via his right femoral artery. The patient had no other complaints, was afebrile, and generally felt well. The clinical impression was either cholesterol emboli versus vasculitis. A $4 \mathrm{~mm}$ punch biopsy was obtained. Histopathology showed a vasculitis with many basophilic organisms consistent with bacteria present within the vascular spaces. There was a perivascular infiltrate consisting of lymphocytes and neutrophils. A gram stain revealed grampositive cocci. The clinician was immediately notified and blood cultures were drawn and echocardiogram performed. The cultures grew staphylococcus aureus. While no evidence of endocarditis was found, a psuedoaneurysm of the right femoral artery was noted. Despite rapid admission and treatment, the patient subsequently expired.

\section{CUTANEOUS METASTASIS: A SINGLE INSTITUTION CLINICAL AND HISTOPATHOLOGIC EXPERIENCE}

D. Sariya, R. Adams-McDonnell, G. Cusack*, X. Xu^, R. Elenitsas*, J. Seykora*, T. Pasha, P. Zhang, M. Baldassano, S. Lessin\# and H. Wu.

Dept. Path. and \#Med. Oncology, Fox Chase Cancer Center, Dept.

*Dermatology and

${ }^{\wedge}$ Path. \&Lab. Medicine, Hospital of the Univ. of Pennsylvania, PA, USA

Forty-four cases of biopsy-proven cutaneous metastases from internal organ or soft tissue malignancies were retrieved at a single institution from 1990 to 2004. All cases had detailed clinical and follow-up data. There were 19 males and 25 females, with a wide age range (37-86 years). Most $(75 \%)$ had advanced (stage IV) disease at the time of metastasis. In four patients skin metastasis was the presenting lesion.
The most common primary site for men is lung and for women, breast and lung. In nearly half of patients cutaneous metastasis was not the clinical diagnosis. The prognosis was grave $(67 \%$ died of disease within a median of 7 months). Independent, blinded review by four dermatopathologists showed that without a detailed clinical history, most metastases from carcinomas were correctly suspected based on histologic patterns. However, metastases from small cell carcinomas and sarcomas were difficult to distinguish from primary skin tumors. Histologic recognition of a primary site was often difficult without pertinent clinical history. None of the metastasis had a combined immunohistochemical profile of B72.3 (-), CA125 (-), calretinin (+), p63 (+) and CK5/6 (+), which was commonly seen in skin adnexal tumors. This immunohistochemical panel appears to best differentiate the two entities.

\section{CONGENITAL ANNULAR ERYTHEMA IN TWO NEWBORNS: SIMILAR MORPHOLOGY, DIVERGENT ETIOLOGIES}

E. Satter, A. Andea, M. Caplan, B. Soder, J. Dodson and J. Metcalf Department of Dermatology and Pathology, Medical University of South Carolina, Charleston, SC, USA

The inconsistent use of the term "erythema annulare centrifugum" has created confusion, in that it has been applied to a group of distinct diseases as if they were a single condition. The literature becomes more ambiguous when different names are given to annular eruptions that have a similar pathogenesis. We present two neonates who presented at birth with similar diffuse erythematous annular eruptions, but differed in their serologic and histopathologic findings. The first child was otherwise healthy and had normal laboratories findings that excluded both infectious and autoimmune diseases. His biopsy showed a perivascular and interstitial infiltrate throughout the entire dermis that was composed of lymphocytes, histiocytes and numerous eosinophils. The second neonate had elevated anti-Ro and anti-La antibodies, thrombocytopenia and a non-anion gap metabolic acidosis. His biopsy showed focal vacuolar degeneration of the basal layer. There was a perivascular and interstitial infiltrate that extended throughout the dermis which consisted primarily of nuclear debris. Within the cytoplasm of the few intact histiocytes and neutrophils, were hematoxylin bodies, giving these cells the appearance of LE cells. We discuss the clinical and histopathological findings in these two neonates and attempt to clarify the nomenclature pertaining to the annular erythemas.

\section{AQUAGENIC SYRINGEAL ACROKERATODERMA}

L.A. Scalf, B. Baldwin and J. Messina

Department of Pathology, University of South Florida, Tampa, FL, USA

A 24 year-old man with a 3-year history of hyperhidrosis presented with intermittent bilateral, burning, whitish translucent patches with excessive wrinkling of the palms. These symptoms occurred within minutes of exposure to water or sweat. He complained of pain with hand flexion during flares. Symptoms usually resolved within an hour of drying. Soles were spared. No relationship to pressure or trauma existed. He had no history of atopy, other skin disease, or family history of a similar disorder. Physical examination revealed several macerated patches in the center of the palms. After immersion in water for several minutes, the palms and lateral fingers became whitish, translucent, edematous, thickened, intensely wrinkled, and painful. There was no response to topical emollients and aluminum chloride. Biopsy revealed hyperkeratosis; the epidermis was otherwise unremarkable. Eccrine ostia were unremarkable. No significant 
inflammation was present. The eccrine epithelium was distinctively hyperplastic and papillated, with focal stratification. Glandular cells were enlarged with abundant, granular cytoplasm. To our knowledge, this is the first reported case of aquagenic syringeal acrokeratoderma in a male patient. All other reported cases have been in women from 9 to 33 years old. The histologic features of the present case are also unique.

\section{VERRUCOUS MALIGNANT MELANOMA WITH LENTIGO MALIGNA MASQUERADING AS INVASIVE SQUAMOUS GELL CARCINOMA \\ L.A. Scalf, J.L. Messina and L.F. Glass \\ Departments of Pathology and Internal Medicine, Division of \\ Dermatology, University of South Florida, Tampa, FL, USA}

An 85 year-old woman presented with two pearly papules on her left cheek. The clinical impression was BCC. Biopsy revealed a proliferation of atypical keratinocytes emanating from the epidermis and extending beyond the papillary dermis. A diagnosis of invasive squamous cell carcinoma was rendered. Excison with frozen section margin control was performed. Permanent sections revealed melanoma, at least $2.06 \mathrm{~mm}$ in depth, with melanoma in situ extending to all peripheral margins. Upon re-examination of the initial biopsies, pseudoepitheliomatous epidermal hyperplasia $(\mathrm{PEH})$ with marked squamous atypia was noted. Intimately admixed with the squamous proliferation was a growth of large malignant epithelioid cells. The epitheliod cells lacked pigment production and extended to the shave biopsy margins. This second population of cells was strongly S-100 positive, diagnostic for amelanotic verrucous melanoma. A revised diagnosis of verrucous malignant melanoma, Clark level III, $0.82 \mathrm{~mm}$ in depth was rendered. The patient's course was complicated by multiple positive surgical margins and she ultimately received XRT. This case spotlights the rare occurrence of $\mathrm{PEH}$ in conjunction with melanoma. PEH can mask the underlying melanoma and lead to a diagnosis of squamous cell carcinoma. As demonstrated, immunostaining is essential in diagnosis of this difficult tumor.

\section{PERIVASCULAR MYOMA OF MYOFIBROMATOSIS AND MYOPERICYTOMA-TYPE ARISING IN A GHRONIC SCAR: A CASE REPORT \\ Rolf Sjuve Scott, Kristie L. Blank*, Liana H. Proffer*, Eric W, Kraus* \\ Josefine Heim-Hall Departments of Pathology and Dermatology*, University of Texas Health Science Center at San Antonio, Texas, USA}

We describe a case of a cutaneous perivascular myoma with features overlapping between the myofibromatosis and the myopericytoma type. The patient is a 58-year-old female with a painless plaque-like and multinodular lesion in the pretibial dermis. She reported repeated trauma to this site, first in her early youth that left an area of hyperpigmentation, and then again at age 40 . She had noticed the appearance of nodules in this area 5 years prior to presentation, which had increased in size and number over the last six months. The biopsy showed a biphasic pattern with a myofibromatosis type component composed of spindle cell myoid nodules and more cellular round cell areas. The myopericytoma-like areas appeared to be infiltrating along vessels. These areas contained aggregates of immature cells arranged concentrically around vascular lumina reminiscent of pericytes. Immunohistochemical stains showed focal positivity for smooth muscle actin. Immunohistochemical and ultrastructural studies have shown these pericyte-like cells to be of a myoid origin. The reason for the neoplastic proliferation of perivascular myoid cells is presently unknown. The association of trauma and neoplastic transformation of the skin is rare. We report the first case of a cutaneous perivascular myoma arising in a chronic scar.

\section{SUBCLINICAL CHRONIC LYMPHOCYTIC LEUKEMIA ASSOCIATED WITH A 13Q DELETION PRESENTING INITIALLY IN THE SKIN: A PROPOS OF A CASE. $\mathrm{K}$ Seilstad $^{1}$, A Khandelwal ${ }^{1}, \mathrm{~K} \mathrm{Gupta}^{1}, \mathrm{~J} \mathrm{Byrd}^{2}$ and C Magro ${ }^{1}$ Department of Pathology ${ }^{1}$ and the Department of Internal Medicine $^{2}$ \\ The Ohio State University, Columbus, OH, USA}

Introduction: B-cell chronic lymphocytic leukemia (B-CLL) represents a low grade $\mathbf{B}$ cell lymphoproliferative disease with overlapping features with small lymphocytic lymphoma. The neoplastic cell is an autoreactive GD5 CD23 B lymphocyte. B-CLL may involve the skin, typically in the context of known disease. We present a case of subclinical B-CLL presenting initially in the skin. Results: A 73year-old male developed a lesion on his right cheek in April 2003 compatible with basal cell carcinoma. The re-excision specimen contained a well differentiated atypical lymphocytic infiltrate consistent with B-CLL along with residual carcinoma. Subsequent laboratory studies revealed slight thrombocytopenia and mild peripheral blood lymphocytosis. A diagnosis was made of stage 0 CLL. Chromosomal studies on peripheral blood showed a deletion at 13q14.3. Conclusion: This case represents a cutaneous presentation of subclinical BCLL. There are two prior reports describing B-CLL presenting initially in the skin. In one case the infiltrates were incidental on a re-excision specimen. The second report suggests $16 \%$ of B-CLL patients have cutaneous manifestations as the first sign of disease. Data indicates that $13 \mathrm{q} 14$ deletion in B-CLL in early stage disease may portend a more aggressive course, possibly explaining its early presentation in the skin in our case.

\section{CD10 AND GD20 EXPRESSION IN A PRIMARY CUTANEOUS LYMPHOMA OF MATURE T CELL CD8 PHENOTYPE.}

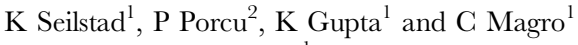

Department of Pathology ${ }^{1}$ and the Department of Internal Medicine $^{2}$,

The Ohio State University, Columbus, OH, USA

Introduction: Most primary cutaneous T cell lymphomas are derived from mature $\mathrm{T}$ lymphocytes. We encountered a case of primary cutaneous $\mathrm{T}$ cell lymphoma with an undescribed phenotypic profile as defined by CD3+, CD8+ lymphocytes that co-expressed CD10, CD20 and CD30. Results: The patient, a 65-year-old woman, presented in 2001 with a thigh mass that was biopsied and held to represent a lymphoblastic T cell lymphoma based on CD10 expression. Two years later the patient developed a recurrence. The pathology showed a large cell lymphoma manifesting a CD8 phenotype although with CD10/CD20 expression. Conclusion: The importance in this case lies in the novel phenotype, namely a mature post thymic $\mathrm{T}$ cell lymphoma exhibiting CD10 and CD20 positivity. CD10 expression in primary cutaneous $\mathrm{T}$ cell lymphoma in the literature is largely limited to two case reports. CD10, a neutral endopeptidase, cleaves proteins with inflammatory or proinflammatory activity released by dying malignant and benign $\mathrm{T}$ cells including those of post thymic origin and may also degrade cytokines which inhibit $\mathrm{T}$ cell apoptosis. Another unique aspect of our case was CD20 positivity whereby only a small number of prior citations exist, all with a CD8 phenotype as was the case in our patient. 
LAMISIL INDUCED DERMATOMYOSITIS

K Seilstad ${ }^{1}$, D Hearne ${ }^{2}$, K Gupta ${ }^{1}$ and C Magro ${ }^{1}$

Department of Pathology ${ }^{1}$ and the Department of Internal

Medicine $^{2}$

The Ohio State University, Columbus, OH, USA

Background: Dermatomyositis, a connective tissue disease syndrome where antibodies to the endothelium of the microvasculature of the skin, muscle and lung are implicated in lesional propagation, is characterized by photodistributed erythema, heliotrope rash, Gottron's papules, muscle weakness and interstitial pulmonary fibrosis. Endotheliotropic viruses and underlying neoplasia are among the inciting triggers. Uncommonly drugs, namely the lipid lowering agents, have been implicated in dermatomyositis. Case Report: The patient, a 57-year-old male, developed a photodistributed rash and muscle weakness following treatment with the antifungal medication Lamisil. A skin biopsy was performed, showing an atrophying interface dermatitis with pandermal mucinosis and striking vasculopathic changes including endothelial cell necrosis with denudement and basement membrane zone reduplication. Direct immunofluorescent testing showed prominent staining of C5b-9 along the dermalepidermal junction and within the vasculature. Conclusion: We have shown a temporal association between use of Lamisil and the development of dermatomyositis. Terbinafine, the active agent in Lamisil, promotes apoptosis of human endothelial cells in culture. Enhanced endothelial cell apoptosis results in the displacement of various cellular antigens creating a state of neo-antigenicity; its attendant sequelae has held to be one of antiendothelial cell antibody formation, a defining pathogenetic event in the evolution of dermatomyositis.

\section{IS IT APPROPRIATE FOR NONDERMATOLOGIST PHYSICIANS TO PERFORM SKIN BIOPSIES?}

K. Sellheyer and W. F. Bergfeld

Departments of Dermatology and Pathology, The Cleveland Clinic Foundation, Cleveland, OH, USA

Physicians of various specialties submit cases for histopathological examination. Using the dermatopathology report as an objective verification tool of clinical diagnostic accuracy we assessed the diagnostic abilities of nondermatologist physicians, who performed various types of skin biopsies, and compared them with those of dermatologists. In total, 4,451 cases were analyzed. Dermatologists diagnosed twice the number of neoplastic and cystic skin lesions correctly $(75 \%)$ than nondermatologists (40\%). The clinical diagnosis rendered by family practitioners matched only in $26 \%$ the histopathological diagnosis. Plastic surgeons did better than family physicians but still had a diagnostic accuracy rate of only $45 \%$ for skin tumors. Inflammatory skin diseases were correctly diagnosed in $71 \%$ of the cases by dermatologists but in only $34 \%$ of the cases by nondermatologists. In our view, it is appropriate for nondermatologists to perform skin biopsies only, if they have required sufficient knowledge in basic dermatology and dermatopathology. A skin biopsy consists not only of the technical aspects of the actual procedure but necessitates an understanding of basic dermatology and dermatopathology to facilitate clinicopathological correlation.

SUBCUTANEOUS PANNICULITIS-LIKE T CELL LYMPHOMA DEVELOPING IN A PATIENT WITH CHRONIC B-CELL LYMPOCYTIC LEUKEMIA

L Shahabi $^{1}$, B Schapiro $^{2}$ and J Cotton ${ }^{2}$

Department of Dermatology ${ }^{1}$, Wayne State University, Detroit, MI and Skin Pathology Services ${ }^{2}$

St. Joseph Mercy Hospital, Ann Arbor, MI, USA
Subcutaneous panniculitis-like T-cell lymphoma (SPTCL) is an unusual peripheral lymphoma most typically presenting with a cytotoxic (CD8-positive, TIA-1-positive) immunophenotype. SPTCLs may have an indolent or highly aggressive clinical course. Histologically, SPTCL may be notoriously difficult to diagnosis. Cases of SPTCL with a deceptively benign appearance similar to that of subcutaneous lupus erythematosus have been described. SPTCL associated with a concomitant systemic leukemia/lymphoma has not been documented in the literature. We report a case of SPTCL arising in a 65-year-old female with a well-established history of B-cell lymphocytic leukemia (BCLL). She presented with two months of recurrent fever and painless erythematous nodules on bilateral lower extremities that were clinically felt to be erythema nodosum. Initial biopsies demonstrated a polymorphous lobular infiltrate with neutrophils, karyorrhexis and lipomembranous change. An excisional biopsy demonstrated an atypical lymphoid population that expressed CD8 and TIA1. PCR analysis confirmed T-cell receptor gene arrangement. The patient was treated with systemic chemotherapy with resolution of her symptoms and complete remission. This is the first well documented case of SPTCL occurring in a patient with long standing B-CLL, and highlights the difficulty of establishing an unequivocal diagnosis of SPTCL.

\section{CUTANEOUS HYALOHYPHOMYCOSIS SECONDARY TO PAECILOMYGES SPEGIES TREATED WITH VORICONAZOLE IN AN IMMUNE COMPENTENT HOST}

H. Skelton, H. Edwards and K. Smith

Department of Dermatopathology, Anatomic Pathology, Quest

Diagnostics, Tucker, GA, USA

Paecilomyces, a hyalohyphomycosis, is an uncommon cause of cutaneous and subcutaneous infections. Most cases reported have occurred in patients with impaired host defenses or following a surgical procedure, and have proven highly resistant to anti fungal therapies. A 60-year-old man presented with scattered non-healing verrucous lesions as well as ulcers and excoriations on the upper extremities and trunk. The lesion had begun shortly after the patient was scratched by prickly okra. The patient had no underlying health problems and he was on no medication. Histology revealed epidermal hyperkeratosis, acanthosis, with papillomatosis with intraepidermal micro-abscess formation. Within the dermis there was a mixed acute and chronic granulomatous infiltrate. With GMS stain numerous pleomorphic yeast forms and pseudohyphael forms were seen. Cultures confirmed Paecilomyces species. The patient was treated with Voriconazole with complete resolution of his lesion. Paecilomyces is an emerging fungal pathogen, which in our immune competent patient manifest as widespread cutaneous disease.

\section{GENETIC IDENTITY ANALYSIS, A PROCEDURE THAT MAY HAVE APPLICATIONS IN CLINICAL PRACTICE}

H. Skelton, W. Sun and K. Smith

Department of Dermatopathology, Anatomic Pathology, Quest

Diagnostics, Tucker, GA.

Genetic identity analysis is a procedure that may be done on paraffin blocks to determine genetic identity or non-identity of tissues. On occasion biopsy specimens from different patients are mixed up. This becomes a major problem if the subsequent diagnoses require further therapy or have other significant medical implications. We recently had such a case in which three biopsy specimens from a middle age man were possibly mixed up with that from a pre-teen age boy. The 
Abstracts

biopsy from the pre-teen was originally reported as a malignant skin tumor. Initially, the three biopsy specimens from the middle age man were sent for genetic identity analysis, which showed that one biopsy specimen was genetically different. The biopsy specimen from the pre-teen was then sent, and found to be genetically similar to the two genetically identical specimens originally submitted for the middle age man. Further confirmation of genetic identity could have been obtained by obtain further samples from both patients, but was not felt necessary in this case. We review the procedure used for genetic identity analysis as well as its other applications for this procedure in clinical practice.

\section{MYOEPITHELIOMA OF THE SKIN WITH P63 EXPRESSION \\ M.J. Smith-Zagone, J.A. Reed and M.R. Schwartz} Department of Pathology, Baylor College of Medicine, Houston, TX, USA

A case of cutaneous myoepithelioma is reported. A 37 year-old woman presented with a $2 \mathrm{~cm}$ well-circumscribed dermal nodule of the forehead. The tumor was enucleated with the clinical diagnosis of an epidermal inclusion cyst. Histologically, the tumor was located within the dermis and was well circumscribed. It was composed of spindle-shaped and epithelioid cells arranged in organoid nests. Focal areas of extracellular hyalinized stroma were present. Well-defined glandular structures, chondroid matrix, and significant nuclear pleomorphism were absent. The tumor expressed widespread cytoplasmic positivity for cytokeratin (using AE1/AE3) and nuclear positivity for p63. Variable reactivity was noted with EMA and S-100. The tumor was negative for smooth muscle actin, GFAP, chromogranin, synaptophysin, and CEA. These immunohistochemical results supported myoepithelial differentiation. Myoepitheliomas of the skin are rare neoplasms that have only recently been recognized in the skin. A single publication has reported the diagnostic utility of p63 in the diagnosis of myoepithelial tumors of the skin. Myoepitheliomas often display variable expression of myoepithelial markers, with no single marker that is $100 \%$ sensitive. The current case highlights the need for a battery of markers, including p63, to detect myoepithelial differentiation.

\section{EOSINOPHILIC NEURITIS AND EOSINOPHILIC PANNICULITIS IN A PATIENT WITH ADVANCED AIDS Anthony C. Soldano, Sean Amsbaugh, and Pamela A. Groben Departments of Surgical Pathology and Dermatology, University of North Carolina Hospitals, Chapel Hill, North Carolina, USA}

A 32-year-old immunocompromised African American male on multiple medications with a history of psoriasis and prurigo nodularis presented with a new generalized, painful, pruritic, papular eruption. Histologic sections revealed epidermal changes of lichen simplex chronicus with superficial and deep perivascular infiltrate consisting of lymphocytes and frequent eosinophils. Two important histological features also identified were eosinophilic panniculitis and prominent perineural eosinophilic infiltrate with focal areas showing eosinophils extending into the substance of the nerve. A right thigh sample was also sent for Gram stain, AFB and fungal cultures, all of which were negative. Laboratory findings at this time were significant for a peripheral blood eosinophilia. The patients' topical corticosteroid prescription was increased with minimal relief. Hypereosinophilic syndrome with dermatoses in HIV infected patients is well documented. We present a patient with advanced AIDS, generalized painful, pruritic, papular rash and peripheral blood eosinophilia. The pattern of inflammation on HIV dermatoses seen in our patient not previously described in the literature is the perineural eosinophilic infiltrate and eosinophilic panniculitis. We propose two possible explanations for these histological findings: (1) hypereosinophilic syndrome in AIDS patients is a heterogeneous disorder and may include eosinophilic neuritis with panniculitis, or (2) an exuberant drug reaction.

\section{GUTANEOUS NEGROTIZING SARCOIDOSIS}

K. Spoonemore, A. Neil Crowson and R. Cornelison

Department of Dermatology, University of Oklahoma, Oklahoma City, OK, USA

A 33-year-old African American woman presented with a 2 year history of skin lesions on her legs. Skin biopsy demonstrated interstitial and focally confluent reticular dermal histiocytic infiltrate consistent with granuloma annulare. She was treated with high-potency topical steroids and returned 14 months later with diffuse papular lesions, $80 \mathrm{lb}$. weight loss, and dyspnea. Repeat skin biopsy showed similar distribution of interstitial and sheet-like histiocytopathy, accompanied by fibrinoid necrosis, cell-poor lymphocytic interface dermatitis and thrombogenic vasculopathy. Stains for fungi $(\mathrm{AB}-$ PAS and GMS) and mycobacterial species (Ziehl-Neelsen, Fite and auramine-rhodamine immunofluorescence) were negative. No foreign material was evident under polarized light. A diagnosis of cutaneous necrotizing sarcoidosis was made. Necrotizing sarcoidosis is a well-recognized pulmonary entity, but is rarely reported in the skin. Diagnostic features include granulomata with regional necrosis, often with a granulomatous vasculitis, in the absence of demonstrable microbial pathogens. Patients are typically adult women who present with pulmonary symptoms, fever, weight loss and fatigue. The etiology of sarcoidosis remains obscure. One proposed mechanism is an id reaction to an as yet unidentified pathogen, with circulating microbial antigens leading to an occlusive and infarctive vasculopathy. Such a mechanism could explain the pathologic changes seen with our patient.

\section{MAST CELL TRYPTASE AND MICROPHTHALMIA TRANSCRIPTION FACTOR EFFECTIVELY DISCRIMINATE BETWEEN GUTANEOUS MAST CELL DISORDERS AND LEUKEMIA CUTIS: A COMPARATIVE CYTOCHEMICAL AND IMMUNOHISTOCHEMICAL STUDY \\ U. Sundram and Y. Natkunam \\ Department of Pathology, Stanford University, Stanford, CA, USA}

Cutaneous mast cell disorders are uncommon but a subset of these disorders, especially mastocytoma, can histologically mimic leukemia cutis. Our objective was to employ a panel of cytochemical and immunohistolochemical markers to determine which markers would be most useful in distinguishing these entities. We studied 17 cases of cutaneous mast cell disorders (UP, TMEP, and mastocytoma) and 12 cases of leukemia cutis (myeloid, myelomonocytic and monocytic) with toluidine blue, Giemsa, pinacyanol erythrocyanate (PE), mast cell tryptase, microphthalmia transcription factor (MITF), CD117, myeloperoxidase, CD43 and CD68. We found that PE stained the majority of cases of mast cell disorders $(11 / 15)$ but did not stain leukemia cutis. Tryptase and MITF stained all cases of mast cell disorders but not leukemia cutis. Although CD117 showed strong staining in all cases of mast cell disorders, it also stained up to $25 \%$ of cases of leukemia cutis (2/8). Staining for myeloperoxidase was specific to leukemia cutis but detected only leukemias with myeloid differentiation $(6 / 12)$. Our results show that mast cell tryptase and 
microphthalmia transcription factor are equally effective in distinguishing mast cell disorders from leukemia cutis. Interestingly, pinacyanol erythrocyanate appears to be specific for mast cell disease, as no staining was seen in leukemia cutis.

\section{CD30-POSITIVE LARGE CELL TRANSFORMATION OF MYCOSIS FUNGOIDES}

Susa JS, High WA, Yegappan S and Hoang MP

Departments of Pathology and Dermatology, University of Texas

Southwestern Medical Center, Dallas, TX, USA

Large cell transformation of mycosis fungoides (MF) is an uncommon phenomenon. We present a case of CD30-positive large cell transformation and discuss its possible pathophysiology. A 74 yearold male with a 36-year history of patch stage MF presented with a 3 -month history of right chest cellulitis that was refractory to IV antibiotic treatment. Skin biopsies from his thigh demonstrated a patchy dermal infiltrate of irregular and hyperchromatic lymphocytes and epidermotropism. The majority of the infiltrate was positive for CD4, CD3, CD2, and negative for CD7. Only 10\% were positive for CD25 and CD8. Biopsies obtained from the ulcerated chest nodules showed a dermal infiltrate of large and pleomorphic lymphoid cells with prominent nucleoli. These large lymphoid cells were strongly positive for CD3, CD30, CD25, CD2 and UCHL-1. Occasional cells were positive for CD4 and CD20. They were negative for ALK-1, TIA-1, CD7, CD8, and CD15. $\mathrm{T}$-gamma receptor gene rearrangement analyses by polymerase chain reaction demonstrated a clonal process with similar rearrangement patterns identified in the patch stage $\mathrm{MF}$ as well as in large cell transformation areas. Examinations of his peripheral blood and bone marrow were negative. The patient had tolerated one cycle of CHOP chemotherapy.

\section{DEEP TRICHOPHYTON RUBRUM INFECTION IN THE IMMUNOCOMPROMISED HOST: AN ENTITY DISTINCT FROM MAJOCGHI'S GRANULOMA \\ Brian L. Swick and Michael J. Messingham \\ Mary Seabury Stone, Department of Dermatology, University of Iowa Hospitals and Clinics, Iowa City, IA, USA}

A 64-year-old white male presented with a three-month history of multiple crusted erythematous nodules on the lower legs. The patient was immunosuppressed on prednisone and tacrolimus secondary to liver transplant. Biopsy revealed a fibrinopurulent crust overlying an interstitial, non-follicular oriented, neutrophilic infiltrate within the dermis. PAS and GMS positive septate hyphae were present within the stratum corneum while yeast-like forms with pseudohyphal projections, suggestive of Blastomyces dermatitidis, were found within the dermis. There was no evidence of follicular rupture or granulomatous inflammation. Subsequent culture grew Trichophyton rubrum confirming the diagnosis of deep dermatophytosis. This case illustrates several histologic differences in deep dermatophyte infection in the immunocompromised host from invasive dermatophyte infection, Majocchi's granuloma, occurring in the immunocompetent patient. In the immunocompromised patient there is typically the following: no evidence of fungal invasion via a ruptured hair follicle, suppurative inflammation as opposed to granulomatous inflammation, and atypical fungal forms consisting of swollen hyphae and round, yeast-like, arthroconidia, as opposed to the slender hyphae seen in Majocchi's granuloma. In addition, because of the clinical appearance of the lesions, immunocompromised state of the patients, and atypical fungal forms seen on biopsy, an initial diagnosis of systemic mycosis is often incorrectly made.
MULTIPLE TRICHOEPITHELIOMAS AND BASAL CELL CARCINOMAS: A NOVEL SYNDROME?

J. Tan, MD, PhD., J. Levitt, MD and R. Phelps, MD

Departments of Pathology and Dermatology, Mount Sinai School of Medicine, New York, NY, USA

We present a case of a sixty-one-year-old Hispanic man with multiple familial trichoepithelioma, and basal cell carcinoma (BCC). Facial papules and nodules appeared around age five, increasing in number with age. Two BCCs were diagnosed and treated elsewhere, one of which prompted enucleation of the left eye and radiation therapy to that area at age fifty. Physical examination showed nodules (0.5 to $2.5 \mathrm{~cm}$ in size) on the scalp, face, ears, arms and the upper back. Biopsies from such tumors demonstrated three histological patterns: pure trichoepithelioma, trichoepithelioma with unequivocal BCC, and basaloid neoplasms of uncertain histogenesis. The trichoepithelioma shows the typical pattern: hair papillae, cystic, retiform basaloid cells and abundant stroma. The BCGs, which were often admixed with the trichoepitheliomas, were nodular type with peripheral palisading, stromal retraction, central confluent necrosis and adenoid features. The basaloid neoplasm of uncertain histogenesis showed cords and nests of basaloid cells without follicular differentiation. Immunohistochemically BCC's areas showed more uniform and stronger staining for BCL-2 than in trichoepithelioma though the latter showed papillary mesenchymal staining. No significant difference was observed for Ber-EP4 and CD34. We conclude this may represent a unique malignant degeneration or a contiguous gene syndrome.

\section{POROKERATOSIS CAUSING CHANGE IN MELANOCYTIC NEVI \\ CT Thompson \\ Department of Dermatology, Oregon Health Sciences University, Portland, OR, USA}

Porokeratosis is an uncommon cause of clinical change in pigmented lesions. We report two cases of so-called "collision" lesions, in which the cause of clinical change in melanocytic nevi was porokeratosis. One biopsy was submitted concurrently with a biopsy from another anatomic site, which was a superficial pattern basal cell carcinoma. Both the melanocytic nevus and the basal cell carcinoma contained coronoid lamellae, focal diminution of the granular layer and subjacent dyskeratotic keratinocytes, consistent with disseminated superficial actinic porokeratosis (DSAP). The melanocytic nevus, from the lower back, was a broad compound melanocytic nevus, associated with elongated, pigmented rete (lentiginous) and small melanocytes in the papillary and superficial reticular dermis (superficial congenital pattern). There was no evidence of external irritation. The second case showed a similar coronoid lamella, focal diminution of the granular layer and dyskeratotic keratinocytes. There was additional parakeratosis away from the parakeratotic column and a lichenoid host response, suggesting external irritation.

\section{SCALP HISTOLOGY IN RAPP-HODGKIN SYNDROME CT. Thompson and J. Roberts}

Department of Dermatology, Oregon Health Sciences University, Portland, OR, USA

We report unique histologic findings in scalp biopsies from two family members with Rapp-Hodgkin syndrome. Rapp-Hodgkin and the related AEC syndrome show a primary scarring alopecia and pili torti. The syndromes, which are related to ectodactyly, ectodermal dysplasia and cleft lip/palate syndrome, have been credited to 
mutations in the TP63 gene. Most notable on biopsy is a primary scarring alopecia and abnormal hair shaft shape. Calcification in the outer root sheath epithelium, the basement membrane of the lower segment and within naked hair shafts is present. Neutrophils and bacterial rods and cocci are adjacent to the hair shaft in the superior segment, associated with dilated follicular ostia. Perifollicular fibrosis is associated with a variety of inflammatory cell types, mostly lymphocytic. One sample shows interfollicular irregular psoriasiform epidermal hyperplasia with parakeratosis, compact orthokeratosis, hypergranulosis, and intraepidermal neutrophils. Follicular architecture is abnormal with abrupt and linear loss of nucleated matrical cells (B fringe of Adamson). The line of Ayber measures $0.30 \mathrm{~mm}$ on average. Hair shafts measure up to $0.16 \mathrm{~mm}$, with variable abnormal cross sectional shape, usually square. Outer root sheath epithelium is disorganized with slight cellular pleomorphism, and the cuticle of the inner and outer root sheath is irregular in thickness.

\section{PRIMARY MALIGNANT MESOTHELIOMA} PRESENTING AS AN UMBILICAL TUMOR

M-M. Tomaszewski and GP Lupton

Department of Dermatopathology, AFIP, Washington, DC, USA

Malignant mesothelioma presenting as a primary umbilical tumor is not reported in the English literature, to our knowledge. We present a 63-year-old woman with a painless and initially skin colored nodule in the umbilical area, which within one month became erythematous. The performed biopsy revealed an infiltrating glandular and ductal proliferation involving the dermis, subcutaneous fat and underlying skeletal muscle embedded in a loose and mucinous stroma. The tumor cells were of medium size with round to oval nuclei and prominent nucleoli. Mitoses were conspicuous. The tumor cells showed a positive reaction for kermix, EMA, CK5/6, calretinin and CK7 and a negative reaction for CK20, CEA, Ber-Ep4, S100 protein, TTF-1, PRP, ERP, and Leu-7. Since calretinin is both a sensitive and specific marker of reactive and neoplastic mesothelial cells, a diagnosis of malignant mesothelioma, epithelioid variant, was made. The performed exploratory laparotomy showed no evidence of malignancy and wide excision of the umbilical area showed no evidence of residual tumor. Follow-up examinations of the patient with repeated CT scans for the past two years were negative for recurrent or metastatic disease. We conclude that this malignancy likely developed in a setting of umbilical abnormalities associated with mesothelial remnants.

\section{IMPACT OF THE POISSON DISTRIBUTION ON LYMPH NODE STAGING OF MALIGNANT MELANOMA}

\section{J. Turner and R. Vollmer}

Department of Pathology, Duke University Medical Center, Durham, NC, USA; Department of Pathology, Durham VA Medical Center, Durham, NC, USA

BACKGROUND: Melanoma patients without lymph node metastases (Stage II) have a variable five-year survival. The influence of falsely negative lymph nodes on this variability is unknown. DESIGN: The Poisson distribution describes the probability of finding no positive lymph nodes when a certain number are examined. Using this model, we analysed lymph node dissection results from 780 patients with melanoma and published survival results for Stage II patients. RESULTS: In the 294 patients without positive nodes, 18\% had fewer than 7 nodes examined. The Poisson probability of finding no positive nodes when 1 to 6 were examined ranged from 0.4 to to 0.8 (mean 0.6). We partitioned survival for stage II into two categories: those with truly negative nodes with a five-year survival of $80 \%$ and those with a five-year survival matching Stage III (46\%). The observed five-year survival of $77 \%$ in Stage II patients implied that the fraction with false negative nodes was also $18 \%$, which corresponded with the subgroup with fewer than 7 nodes examined. CONCLUSION: The Poisson model suggests that the variable survival in Stage II patients may reflect the incorrect staging of patients with stage III melanoma who have limited numbers of lymph nodes examined.

\section{PATIENTS WITH COMPLETE DIGEORGE SYNDROME AND GUTANEOUS MANIFESTATIONS: A HISTOPATHOLOGIC AND IMMUNOHISTOCHEMICAL STUDY \\ J Turner, ML Markert, J Burchette, C Herman and MA Selim}

BACKGROUND: DiGeorge Syndrome is a congenital anomaly with a constellation of findings including thymic hypoplasia. Approximately 1 in 250 patients has complete DiGeorge syndrome with athymia. Many athymic patients develop severe eczema/ erythroderma associated with oligoclonal circulating $\mathrm{T}$ cells. DESIGN: Nine biopsies from eight athymic patients with severe rash and circulating oligoclonal T cells were studied. Immunohistochemistry with CD3, CD4, CD8 and TIA-1 was performed. RESULTS: The stratum corneum showed parakeratosis (78\%), most commonly confluent. Neutrophilic abscesses were commonly identified $(44 \%)$. The epidermis exhibited exocytosis (100\%), spongiosis $(89 \%)$, dyskeratosis $(89 \%)$, acanthosis $(56 \%)$, and satellitosis $(44 \%)$. Dermal changes included perivascular $(56 \%)$, perifollicular $(33 \%)$, and perieccrine (11\%) lymphocytic infiltrates. Eosinophils were identified $(78 \%)$; most commonly in both the dermis and epidermis. The majority of lymphocytes were CD3 positive with a slight CD4 over CD8 predominance in the dermis and epidermis. $67 \%$ of cases contained TIA-1 positive cells. CONCLUSION: Skin lesions from patients with complete DiGeorge syndrome who develop a rash that clinically resembles severe eczema/erythroderma display a constellation of histologic findings. The presence of dyskeratinocytes, satellitosis, and parakeratotic scale with neutrophils distinguishes these lesions from eczema and other spongiotic disorders. The pathophysiologic role of the inflammatory cells in this entity requires additional study.

\section{HUMAN HERPESVIRUS-8: A USEFUL MARKER FOR DISTINGUISHING KAPOSI SARCOMA AND KAPOSI SARCOMA-LIKE PYOGENIC GRANULOMA \\ A. Uzieblo and S. Kohler \\ Department of Pathology, Stanford University Medical Center, Stanford, CA, USA}

Kaposi's sarcoma (KS) is a vascular neoplasm associated with human herpesvirus-8 (HHV-8) infection. On occasion, KS may histologically mimic pyogenic granulomas (PG), a common benign vascular tumor of the skin. Using immunoperoxidase stains, we examined 28 PG and 4 PG-like KS for HHV-8 to determine the specificity of positive staining in this setting. All PG-like KS demonstrated nuclear staining for HHV-8. No staining was identified in any of the PG. Furthermore, histologic criteria often used to differentiate between these two entities were not helpful in difficult cases. The only distinguishing features were the presence/absence of HHV-8 staining and, in some cases, clinical history. The presence of HHV-8 nuclear staining appears to be a specific marker for KS when comparing PG and PG-like KS. Given the lack of distinguishing morphologic criteria, we suggest performing immunoperoxidase stains for $\mathrm{HHV}-8$ on any $\mathrm{PG}$ occurring in a clinically atypical setting. 


\section{HYALINE CELL-RICH CHONDROID SYRINGOMA-} REPORT OF TWO CASES.

R. Veeramachaneni, I. D. Sanusi and M. Li

Department of Pathology, Louisiana State University Health Sciences Center, Shreveport, LA, USA

Case 1: A 38 year-old man presented with a painless right post auricular mass, slowly growing for ten years. Clinical impression was a salivary gland lesion. The excised mass measured $3 \mathrm{~cm}$ and was gelatinous. Case 2: A 28 year-old man presented with a lump in the region of the left heel, slowly growing for several years. Excision revealed a lobulated pink-gray mass measuring $2 \mathrm{~cm}$. In both cases, histology demonstrated a circumscribed encapsulated proliferation of tumour cells surrounded by abundant myxoid stroma. There were almost exclusively hyaline cells with plasmacytoid features, arranged predominantly in nests and sheets with abundant pink cytoplasm and hyaline inclusions. Round to oval nuclei with occasional small nucleoli and rare mitotic figures were seen. Both cases stained strongly positive for low molecular weight cytokeratin, S 100 and vimentin and were negative for desmin, smooth-muscle actin and muscle-specific actin. We describe two cases of hyaline cell-rich chondroid syringoma, a histologically benign entity. The importance in recognizing this entity is that these tumors can easily be confused with malignant neoplasms including malignant melanoma, myxoid chondrosarcoma and alveolar soft part sarcoma. Follow up for a year has shown no evidence of metastases or recurrence in both cases.

\section{HERPETIC CYTOPATHIC FEATURES CONFINED TO FOLLICULOSEBACEOUS UNITS: WHAT DOES IT MEAN?}

N Walsh, R Boutilier, D Glasgow and M Shaffelburg

Departments of Pathology, Capital District Health Authority and Dalhousie University, Halifax, Colchester Regional Hospital, Truro, Cape Breton Regional Hospital, Sydney and Department of Dermatology, Va

The histopathological changes of herpes simplex, zoster and varicella are considered to be indistinguishable from one another. The clinical setting with adjunctive studies generally clarifies the diagnosis. Vesicular lesions in all 3 conditions can involve epidermal and adnexal epithelium with characteristic cytopathic features. We describe 3 patients with non-vesicular eruptions on the head and neck whose biopsies revealed exclusive folliculosebaceous involvement by herpes. All three patients developed typical herpes zoster within days of the biopsy. There is compelling scientific evidence in the literature indicating that, in herpes zoster, the virus is transported from dorsal root or trigeminal ganglia via myelinated nerves to the skin. These terminate at the isthmus of hair follicles and primary infection of follicular and sebaceous epithelium occurs. Secondary spread of infection to the epidermis follows. In contrast, data pertaining to recurrent herpes simplex indicates that axonal transport of the virus from sensory ganglia to the skin is directed primarily to the epidermis, via terminal nonmyelinated nerve twigs. The clinical evolution of our 3 cases and scientific data in the literature indicate that exclusive folliculosebaceous involvement by herpes, in the setting of a non-vesicular eruption, represents early herpes zoster.

\section{PARACOLLOID OF THE SKIN}

C. Wang, S. Novick and A. R. Wang

Departments of Pathology and Dermatology, Tulane University, New Orleans, LA

The concept of paracolloid was raised by Dupre etal. in 1979 to describe a rare lesion, in which large amounts of homogeneous, pale pink, non-amyloid material in sun-exposed skin. The deposits consisted of haphazardly arranged microfilaments of 3-4 nm in diameter. Here we report another case. A 54-year-old white male presented with a $2 \times 2 \mathrm{~cm}$ boggy, erythematous plaque involving the bridge and tip of the nose for two years. The lesion enlarged slowly and intermittently drained pinkish fluid. A shave biopsy revealed extensive homogeneous, pale pink material with fissure and clefts in the dermis. The deposits wrapped up hair follicles and sebaceous glands, and superficially mixed with scattered solar elastic fibers and globules. There were scattered fibroblasts, focal mild perivascular and perifollicular lymphocyte and plasma cells. Adjacent skin showed prominent solar elastosis. A thin grenz zone was present abutting an atrophic epidermis with actinic keratosis. The deposits showed faint Congo red stain with focal weak apple-green birefringence, faint PAS stain, and negative Verhoeff-van Gieson stain. Masson's trichrome stain and immunohistochemical stain for collagen IV were negative.

\section{RACCOON HEARTWORM CAUSING A LEG MASS}

M.L. Warthan, M.M. Warthan and J. Lesher

Department of Internal Medicine, Section of Dermatology, Medical College of Georgia, Augusta, Georgia and University of Texas Medical Branch, Department of Dermatology, Galveston, TX, USA

A 29-year-old man from east texas presented with a 3-month history of a large, isolated, painful subcutaneous nodule on his lower right leg. An excisional biopsy showed zones of necrosis in the epidermis, dermis, and subcutaneous adipose tissue with a neutrophilic infiltrate. The dermis showed a mixed infiltrate with necrotic debris and a nongravid adult female filarial nematode with a thick, multi-layered cuticle, paired uteri, and an intestine. The histologic features were diagnostic for dirofilaria tenuis, the raccoon heartworm. Dirofilaria tenuis causes heartworm infection in raccoons and infrequently causes human disease. When humans do contract dirofilariasis, the mode of transmission is via a bite from a mosquito infected with dirofilaria larvae. Humans are a dead end host and dirofilaria cannot reproduce in humans, but they do cause various lesions at the site of the mosquito bites. We report a very rare case of human dirofilaria tenuis infection manifesting as a subcutaneous nodule. Dirofilaria tenuis infections are very rare in the U.S., and usually occur in the Southeastern U.S.

\section{CD99 IMMUNOREACTIVITY IN METASTATIC MALIGNANT MELANOMA}

AE Wilkerson, MA Glasgow and KM Hiatt

Department of Pathology, University of Arkansas for Medical Sciences, Little Rock, AR

CD99, also known as p30/32, is a glycoprotein product of the MIC2 gene, which is located on the short arm of both chromosome $\mathrm{X}$ and $\mathrm{Y}$. This transmembrane protein was originally utilized in immunohistochemistry as a unique marker for Ewing sarcoma, other primitive neuroectodermal tumors, and more recently in a wide variety of tumors. It's expression in malignant melanoma (MM) has not been well documented. A recent study at our institution demonstrated membranous staining in approximately $61 \%$ of primary MM. As CD99 is expressed by hematopoeitic cells, it has been proposed as a mechanism for lymphocytes to gain access to the vasculature. 1 This study is designed to determine if CD99 expression in melanoma cells has a similar role using cases of metastatic MM from our archives. Our evaluation shows that 13 of 28 cases $(46.4 \%)$ demonstrated membranous CD99 staining. A case of this magnitude has not been previously reported.Â Reference: 1. Shenkel AR, Mamdouh Z, 
Chen X, Liebman RM, Muller WA. CD99 plays a major role in the migration of monocytes through endothelial junctions. Nature Immunol 2002;3:143-150.

\section{NODULAR VASCULTITIS IN ASSOCIATION WITH PRIMARY BILIARY CIRRHOSIS \\ H. Winfield, A. Rosenberg and B. Smoller \\ Department of Dermatopathology, University of Arkansas for Medical Sciences, AR, USA}

BACKGROUND: Nodular vasculitis is a disorder that can be associated with several disease states, including infection, medications, malignancy, and collagen vascular disorders. The pathogenesis is unknown, but may be related to immune complex deposition, leading to subsequent vascular damage. METHODS: We report a case of nodular vasculitis occurring in a 40-year-old African-American woman with end-stage liver disease and a 3 month history of ulcerating lesions on her lower extremities. Although these lesions were both clinically and histologically consistent with erythema indratum, all studies directed at determining a mycobacterial etiology were negative. RESULTS: Biopsy of the skin lesions revealed a neutrophil-predominant vasculitis in the setting of a lobular panniculitis. The histopathology of the liver biopsies, and the patient's clinical course were consistent with auto-immune cholangitis. A course of oral prednisone was initiated, which resulted in improvement of the lower extremity lesions. CONCLUSION: In this case report we document a case of nodular vasculitis occurring in the setting of auto-immune cholangitis. The pathogenesis of this condition remains obscure, however possible causes include immune complex deposition or a delayed-type hypersensitivity reaction.

\section{CUTANEOUS MYOEPITHELIAL CARCINOMA IN A NEONATE \\ E. Wiser, L. Owen and J. Junkins-Hopkins \\ Department of Dermatology, University of Pennsylvania Health Center, Philadelphia, PA USA}

A 27-day-old female neonate presented with a bleeding growth on the abdomen. The clinical differential diagnosis included pyogenic granuloma and hemangioma. Histology shows an ulcerated, spindled and round cell tumor in the dermis with ill-defined margins that invade the subcutaneous fat. The tumor exhibits a biphasic growth pattern with plump spindled cells arranged in fascicles, as well as epithelioid cells arranged in sheets. Some cells show plasmacytoid features. The tumor cells have pleomorphic, vesicular nuclei. Single cell necrosis and mitotic activity is prominent. Myxoid and chondroid stromal changes are focally seen. Immunohistochemical stains show strong positivity for $\mathrm{S} 100$ protein, calponin, and, focally, AE1/AE3. Portions of the both the spindled and epithelioid cell components show staining with EMA. Desmin and MSA stains were negative. The tumor does not stain for CD 31, CD 30, CD 45, CD 20, CD 34, CK 20, MART-1, or HMB45. These histologic features are consistent with a cutaneous myoepithelial carcinoma. Cutaneous myoepitheliomas are rare tumors of myoepithelial cells which surround eccrine and apocrine glands. While most myoepitheliomas are benign, tumors with high mitotic activity may have a risk for recurrence or metastasis. This is, to our knowledge, the youngest patient described with myoepithelial carcinoma.

\section{RICKETTSIA AFRICAE, A TICK-BORNE PATHOGEN IN TRAVELERS TO SUB-SAHARAN AFRICA}

A. Witkiewicz, J. Shupack and S. Meehan

Department of Dermatology, New York University School of

Medicine, New York, NY
A 31 year-old female presented with fever, arthralgias and myalgia ten days after returning from Zimbabwe. On physical exam she had scattered small, pink papules over the lower abdomen and two hemorrhagic pustules with central necrosis (eschars) on her left leg and left buttock. There was inquinal lymphadenopathy with no splenomegaly or hepatomegaly. A punch biopsy was performed which showed necrotizing vasculitis with ulceration of overlying epidermis and papillary dermis. Immunohistochemical studies performed at the Center for Disease Control and Prevention were positive for spotted group Rickettsiae. Treatment with doxycycline $200 \mathrm{mg} / \mathrm{d}$ for two weeks resulted in complete recovery. The clinical and histopathologic findings were consistent with Tick Bite Fever caused by Rickettsia africae.

\section{ANAPLASTIC LARGE GELL LYMPHOMA} MASQUERADING AS AN INFECTIOUS SYNDROME G. Wolgamot, A. Chien, S. Kussick, J. Olerud, and Z. Argenyi Departments of Dermatology and Pathology, University of Washington, Seattle, WA, USA

The clinical manifestations of anaplastic large cell lymphoma can be remarkably deceptive. A 41-year-old female presented with fever, productive cough, fatigue, arthralgias, and lymphadenopathy. She had been camping in the Southwest, and had visited some bat caves. At the same time, her son developed a similar illness. The patient's white cell count was 23,000, and radiology demonstrated diffuse bilateral infiltrates. Extensive workup for bacterial and fungal organisms were noncontributory, however, a viral exposure could not be excluded. The patient subsequently developed an umbilicated vesicular rash on her torso. Initial skin biopsies showed a perivascular mixed infiltrate, with no evidence of microorganisms. After clinical progression, a subsequent skin biopsy demonstrated a perivascular infiltrate of large mononuclear cells, with vesicular nuclei and abundant cytoplasm, immunophenotypically characteristic of anaplastic large cell lymphoma. With elucidation of the process, lymphomatous involvement of the blood, pleural fluid, CSF, and nodal tissue was identified. Despite chemotherapy, the patient passed away. Exposure to an infectious agent may have unmasked the patient's lymphoma.

\section{A VIRTUAL MICROSCOPY IMAGE DATABASE FOR DERMATOPATHOLOGY EDUCATION.}

\section{J. Woosley}

Departments of Pathology and Laboratory Medicine and

Dermatology, University of North Carolina School of Medicine, Chapel Hill, NG, (USA)

A comprehensive collection of high quality histologic slides is essential for education of pathology and dermatology trainees in dermatopathology. However, maintenance of the quality of the teaching slide collection is constantly challenged by slide loss, breakage, and stain fading. Recut replacement slides are not always easily attainable. When they are, the most important diagnostic features may not be as well represented in recuts. The ScanScope (Aperio Technologies) virtual microscopy instrument and software have been used to create a dermatopathology image database. Histologic sections on glass microscope slides are scanned at X20 to obtain a high-resolution digital image of the entire section (virtual slides). This magnification is optimal for demonstrating most cutaneous pathologic processes, where low or intermediate power pattern recognition is of primary diagnostic importance. Images can be viewed with the ImageScope virtual slide viewing software. The software is intuitive to use and closely mimics viewing of a slide at the microscope. Virtual slide 
image quality is equivalent to that seen at the microscope. Unique teaching slides can be reproduced in unlimited quantities and slides are never lost, broken, or misfiled. Internet, intranet, or DVD access permits continuous availability of a teaching slide collection to trainees.

\section{USE OF SCREEN RECORDING SOFTWARE TO CREATE VIDEO TUTORIALS FOR DERMATOPATHOLOGY EDUCATION \\ J. Woosley \\ Departments of Pathology and Laboratory Medicine and Dermatology, University of North Carolina School of Medicine, Chapel Hill, NC}

Texts and atlases have limitations when used to teach dermatopathology to trainees: they can be tedious and time-consuming to use, image detail is limited, text and figure legends can be ambiguous, and only a minor degree of image annotation (letters, arrows, lines outlining important features) is possible. A video screen recording software program (Camtasia Studio) has been used to create high quality digital audio and video dermatopathology tutorials. The screen recording program is started with the image window being recorded. Audio narration (USB digital microphone) is recorded along with live drawings of annotating lines outlining important histologic features, creating the effect of a "digital chalkboard" being used by the instructor. Separate clips of each "chalkboard annotation" with accompanying narration can be combined to create a seamless movie detailing each of the important histopathologic features leading to a specific dermatopathologic diagnosis. Video dermatopathology tutorials are extremely effective because they closely mimic instruction at the microscope. However, they offer the significant advantage of allowing remote teaching at any time or place convenient for the learner. Tutorials can be compressed into a variety of multimedia formats and streamed for web delivery or can be provided on $\mathrm{CD}$ for distribution.

\section{CUTANEOUS AND SYSTEMIC PLASMACYTOSIS}

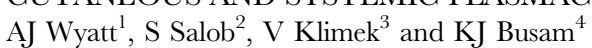

Department of Dermatology, Weill Medical College of Cornell

University $^{1}$, Dermatology Service ${ }^{2}$

Department of Medicine ${ }^{3}$

and Department of Pathology ${ }^{4}$ and

Memorial Sloan-Kettering Cancer Center, NY, NY USA

Background: Plasmacytosis is a rare entity, which may manifest with cutaneous patches and plaques and may subsequently progress to involve extracutaneous sites. First described in Japan by Kimura K et al in 1983, most cases have been reported in patients of Asian descent. Observation: We report a case of plasmacytosis in a 52-yearold Hispanic woman, who presented ten years ago with cutaneous infiltrates of plasma cells and polyclonal hypergammaglobulinemia. Numerous (immunohistochemical, molecular, serologic) studies failed to demonstrate a clonal lymphoplasmacellular neoplasm, collagen vascular disease or chronic infection. Her course has been slowly progressive with new plaques developing on the integument. Plasma cells have since infiltrated gastric and esophageal mucosa, the left temporal artery and lymph nodes. The disease has been resistant to various therapeutic approaches. Conclusions: Our case provides longterm follow-up of a patient with plasmacytosis and illustrates the chronic progressive course of this disease over a 10-year course with unique involvement of the temporal artery and gastrointestinal mucosa.

\section{HYPERTROPHY OF PACINIAN GORPUSCLES IN A YOUNG PATIENT WITH NEUROFIBROMATOSIS}

Shaofeng Yan and Brent T Harris

Department of Pathology, Dartmouth-Hitchcock Medical Center, Lebanon, New Hampshire, USA

A 24-year-old female with a lifelong history of neurofibromatosis type 1 presented with left ring finger pain and decreased sensation for over a year. She has multiple café au lait spots over her entire trunk, upper and lower extremities. She also had 11 surgeries to excise superficial neurofibromas over her body. She first noticed a painful swelling of the proximal phalanx of the left ring finger. She then gradually noticed a loss of sensation distally and a constant numb and tingling feeling distal to the palm and the left ring finger. The clinical impression was neurofibroma. MRI of the tender lesion found no evidence of neurofibroma. Biopsy of the lesion showed hypertrophy of Pacinian Corpuscles. Hypertrophy of Pacinian Corpuscles is a rare condition which has been reported very infrequently. It should be considered in the differential diagnosis of any patient with persistent pain localized to the distal palm or digits in addition to glomus tumor and neuroma. It was thought not to be connected with neurofibromatosis. The finding of this lesion in a patient with neurofibromatosis is somewhat intriguing and the relationship of these two entities is unclear.

\section{BALLOON CELL MELANOMA: CASE REPORT}

Anna Yemelyanova and Elizabeth M. Dugan

Departments of Pathology and Dermatology, Washington Hospital Center, Washington, DC

Balloon cell melanoma (BCM) is a rare histological variant of amelanotic melanoma. The differential diagnosis of clear cell lesion includes BCM, renal cell carcinoma, clear cell sarcoma as well as a number of histiocytic and infectious processes. We report a case of BCM in a 33 year-old Indonesian man who presented with a non-tender, freely movable, non-pigmented left thigh nodule, which had been present for a year. On microscopic examination tumor consisted of a nodular infiltrate present within the dermis with superficial invasion of subcutaneous fat. Tumor cells formed nested aggregates were relatively bland in appearance and had abundant, clear cytoplasm, enlarged nuclei and prominent eosinophilic nucleoli. Rare mitotic figures were present within the deep portion of the neoplasm. No definitive nests were seen at the dermal epidermal junction or within the epidermis. Histochemical stains AFB, PAS/D, and GMS were negative. Immunoperoxidase studies were positive with antibodies to vimentin, S100, HMB45, CD68, and negative with cytokeratin, Factor XIIIA, and lysozyme. The diagnosis of BCMM was favored based on histological features, and immunoperoxidase staining pattern. We believe this case can provide additional information to help establish diagnostic criteria of this rare variant of melanoma.

\section{PAINFUL SUBUNGAL DYSKERATOTIC TUMORS IN INCONTINENTIA PIGMENTI}

A. Young, P. Manolson, B. Cohen, M. Klapper and T. Barrett Department of Dermatology, The Johns Hopkins Medical Institutions, Baltimore, MD, USA

A 65-year-old woman presented with multiple subungal lesions for the past forty years. These were associated with increasing pain with growth, relieved only by destruction. They were treated as subungal warts by electrocautery and surgical excision. A biopsy of one lesion was interpreted as squamous cell carcinoma with subsequent partial amputation of the affected digit. She continued to develop painful subungal growths involving all her fingers, requiring repeated 
surgeries. On physical examination, subungal tumors associated with hyperkeratosis and erythema of the nail bed were found on the right thumb and ring finger. Subungal hyperkeratosis involved all other fingers. On histological examination, there was marked dyskeratosis with features of subungal keratoacanthoma or squamous cell carcinoma. This patient has a history of incontinentia pigmenti (IP) since childhood and she has three daughters with IP. We report a case of painful subungal dyskeratotic tumors in IP, considered as keratoacanthomas by some, as they grow rapidly and may demonstrate spontaneous resolution, albeit rarely. The distinguishing features of subungal tumor in IP are its occurrence in young women with multiple lesions and signs of IP, and its tendency to erupt persistently over a course of several years.

\section{PROGRESSIVE MUGINOUS HISTIOCYTOSIS}

A. Young, J. Olivere, S. Yoo, C. Martins and T. Barrett Department of Dermatology, The Johns Hopkins Medical Institutions, Baltimore, MD, USA

Progressive mucinous histiocytosis is a rare, benign, non-Langerhans cell histiocytosis limited to the skin. A total of ten cases, all women, in four families, and one sporadic case have been described in the English literature. The disorder usually begins in childhood and progresses slowly. We report two sporadic cases of adult-onset progressive mucinous histiocytosis in unrelated African American women, age 48 and 55 years old respectively, who developed redbrown and flesh-colored, asymptomatic papules on the face, arms and legs without truncal, mucosal or visceral involvement. The lesions show no spontaneous regression. Both patients lack associated systemic symptoms including polyuria, polydipsia or seizures. There is no underlying hyperlipidemia, paraproteinemia or lymphoproliferative disease. No family history of similar lesions can be identified. Light microscopy reveals dermal proliferation of spindle-shaped histiocytes with abundant mucin deposition. Electron microscopy demonstrates a high number of myelin-figures or zebra bodies in the cytoplasm of histiocytes. On immunohistochemistry, positive staining with macrophage markers CD68, HAM56 and lysozyme, and Factor XIIIa, a transglutaminase present in dermal dendrocytes, and negative staining with Langerhans cell markers CDla and S100, and CD34, a marker present in dermal dendritic cells derived from uncommitted mesenchymal cells, are observed.

\section{KIKUCHI-FUJIMOTO'S DISEASE WITH SKIN MANIFESTATIONS}

I.T. Yu and M. Fung

Department of Pathology and Department of Dermatology, University of California Davis Medical Center, Sacramento, CA, USA

A fourteen-year-old female presented with persistent fever, abdominal pain, splenomegaly and multiple intra-abdominal lymphadenopathies. She underwent an exploratory laparotomy. Biopsies of the mesenteric lymph nodes revealed necrotizing histiocytic lymphadenitis, characterized by para-cortical fibrinous necrosis with karyorrhectic debris and histiocytic palisading, and paucity of the polymorphic neutrophils. She also developed concurrent skin lesions with scattered small non-itching vesicles and blisters over her back and lower legs. A punch biopsy revealed vacuolar interface changes, sub-epidermal blister formation, mild superficial perivascular infiltration, and follicle necrosis. Karyorrhectic debris and CD68-positive cells are noted around the follicle. A direct immunofluorescent study was negative for complement or immunoglobulin depositions. The patient was diagnosed as Kikuchi-Fujimoto's disease, or necrotizing histiocytic lymphadenitis, a benign self-limited lymph node disorder most commonly occurred in young females. Patients with Kikuchi's disease are typically presented with isolated cervical lymphadenopathy with or without accompanying fever. Multiple lymph node involvement, splenomegaly and/or cutaneous lesions are rare occurrences. The present patient experienced a severe inflammatory response syndrome manifested by respiratory failure, pancytopenia, and disseminated intravascular coagulopathy. She recovered completely after two weeks of intensive medical treatment. She has had no respiratory symptoms, residual lymphadenopathies or documented skin lesions in her follow-up three months after discharge.

\section{ATYPICAL FIBROXANTHOMA WITH GRANULAR CELL CHANGE \\ P. Zeikus, U.Pajvani, M. Kuperman, G. Telang and L. Robinson- Bostom \\ Department of Dermatology, Brown Medical School, Providence, RI, USA}

Atypical fibroxanthoma is a fairly common dermal neoplasm that is characterized histologically by infiltrating pleomorphic spindle cells and atypical giant cells with numerous mitoses. Clinically, the lesion most commonly presents as a solitary ulcerated papule on the head or neck of elderly males. We report a patient with a rare granular cell variant of atypical fibroxanthoma. The patient was a seventy-five-year-old male with a history of squamous cell carcinoma on the left scalp treated by Mohs surgery two years prior. In the vicinity of the previous squamous cell carcinoma site, the patient presented with a slow growing, approximately 1.0 by $1.0 \mathrm{~cm}$ red, violaceous, firm nodule on the left scalp. The clinical impression was recurrent squamous cell carcinoma. Biopsy of the lesion revealed a dermal tumor composed of pleomorphic cells with eosinophilic granular cytoplasm with numerous typical and atypical mitoses. The tumor stained focally with Factor VIII, CD 68, and SMA and $30 \%$ of the neoplastic cells stained with Ki 67. Staining for Keratin cocktail, High Molecular Weight Keratin, Melan A and S100 were negative. PAS strongly stained the granules. These features are consistent with a granular cell variant of atypical fibroxanthoma.

\section{HTERT EXPRESSION IN MELANOCYTIC LESIONS: AN IMMUNOHISTOCHEMICAL STUDY ON PARAFFIN- EMBEDDED TISSUES \\ W. Zhu, L. Su, D. Thomas and D. Fullen}

Telomerase plays a role in the immortalization of cells and carcinogenesis. Previous studies have yielded conflicting results on whether human telomerase RNA (hTER) expression differs in nevi, atypical nevi and melanomas using PGR-based telomeric repeat amplification protocol (TRAP) or in situ hybridization assays. The aim of this study was to evaluate human telomerase reverse transcriptase (hTERT) staining in melanocytic lesions on paraffin-embedded tissues. Paraffin-embedded sections from 6 acquired nevi, 4 dysplastic nevi, 5 Spitz nevi, 6 primary invasive melanomas, and 3 metastatic melanomas, were studied for staining intensity $(0-3+)$ and $\%$ of labeled cells with anti-hTERT. HTERT staining was observed in most cells $(>75 \%)$ in all lesions, and was of greater intensity in the nucleus, especially the nucleolus, compared to the cytoplasm. Spitz nevi tended to have weaker hTERT staining $($ mean $=1.2)$ compared to acquired nevi $($ mean $=2.0)$, dysplastic nevi $($ mean $=2.5)$, primary melanoma $($ mean $=2.17)$, or metastatic melanoma $($ mean $=3)$. Although telomerase activity was weaker in Spitz nevi, there was overlap with other nevi and primary invasive melanomas in our small series. Thus, hTERT expression does not appear to be a reliable adjunct to the histological diagnosis of primary melanocytic lesions. 